\title{
Ultrafast Resonance Energy Transfer in Ethylene-Bridged BODIPY Heterooligomers: from Frenkel to Förster Coupling Limit
}

\author{
Lukas J. Patalag, , Joscha Hoche, Marco Holzapfel, Alexander Schmiedel, Roland Mitric,* \\ Christoph Lambert, ${ }^{*}$ Daniel B. Werz ${ }^{*}$
}

\section{Supporting Information}

Table of Contents

1. General Information $\quad$ S2

2. Spectroscopic Data of Prepared Final Compounds $\quad$ S3

3. Building Blocks, Products and Synthetic Procedures S5

3.1 Products and synthetic procedures for BODIPY monomers and DM homooligomers S5

3.2 Products obtained by hetero- and homo-oligomerizations and general synthetic procedures: 2G| $\left.\right|^{\mathrm{Et}}$, 3G $\left.\right|^{\mathrm{Et}},\left.\mathbf{4 G}\right|^{\mathrm{Et}}, 2 \mathrm{G}-$ Red, 2G-Red $\left.\right|^{(\mathrm{Et})} \quad$ S8

3.3 Absorption and fluorescence spectra of homooligomers 2G $\left.\right|^{\mathrm{Et}},\left.\mathbf{3 G}\right|^{\mathrm{Et}}$ and $\left.\mathbf{4 G}\right|^{\mathrm{Et}} \quad$ S13

3.4 Products obtained by Knoevenagel condensations and general synthetic procedure:

Red, Red $\left.\right|^{\mathrm{Et}}$, 1G-Red, 1G-Red $\left.\right|^{(\mathrm{Et})}$, 3G-Red, 3G-Red $\left.\right|^{(\mathrm{Et})}$

4. Calculation of Transition Dipole Moments $\quad$ S26

$\begin{array}{ll}\text { 5. Transient Absorption Spectra } & \text { S27 }\end{array}$

6. Computational Details $\quad$ S33

6.1 Conformational analysis:

Computational archive for competing minimum superstructures of all heterooligomers $\quad$ S33

$\begin{array}{lll}\mathbf{6 . 2} & \text { Energy transfer rates } & \mathbf{S 4 7}\end{array}$

6.3 FRET rates according to traditional formula $\quad \mathbf{S 5 0}$

$\begin{array}{lll}\text { 6.4 Field-induced surface hopping dynamics simulations } & \text { S51 }\end{array}$

7. References 


\section{General Information}

All solvents were purchased as HPLC grade solvents and stored under molecular sieves. All reactions were carried out in oven-dried glassware, septum-capped under atmospheric pressure of argon. Commercially available compounds were used without further purification unless otherwise stated.

Proton $\left({ }^{1} \mathrm{H}\right)$, carbon $\left({ }^{13} \mathrm{C}\right)$ and fluorine $\left({ }^{19} \mathrm{~F}\right)$ NMR spectra were recorded on a Bruker Avance II 300, a Bruker DRX 400, a Bruker Avance III 400, and a Bruker Avance II 600 spectrometer using the residual signals from $\mathrm{CHCl}_{3}, \delta 7.26 \mathrm{ppm}$ and $\delta 77.2 \mathrm{ppm}$ and $\mathrm{SiMe}_{4}, \delta 0.00 \mathrm{ppm}$ as internal references for ${ }^{1} \mathrm{H}$ and ${ }^{13} \mathrm{C}$ NMR chemical shifts, respectively. The following abbreviations were used to explain the multiplicities: $\mathrm{s}=$ singlet, $\mathrm{s}_{\mathrm{br}}=\mathrm{broad}$ singlet, $\mathrm{d}=$ doublet $\mathrm{t}=$ triplet $\mathrm{q}=$ quintet, $\mathrm{m}=$ multiplet.

ESI-HRMS mass spectrometry was carried out on a FTICR instrument.

IR spectra were measured on an ATR spectrometer.

Steady-State Absorption Spectroscopy. The UV/vis/NIR absorption spectra were measured in $1 \mathrm{~cm}$ quartz cuvettes from Hellma using a Cary 5000 spectrometer. The pure solvent was used as reference.

Emission Spectroscopy. Measurements were performed with an Edinburgh Instruments FLS980 spectrometer. Fluorescence quantum yields were measured with an integrating sphere and the FLS980 spectrometer. The corresponding sample was measured at various dilutions to approach a value without self-quenching contributions. Each measurement was repeated three times and the average was calculated. Fluorescence lifetimes were determined by time-correlated single-photon counting (TCSPC) with the FLS980 spectrometer by exciting the samples with pulsed laser diodes at at $23900 \mathrm{~cm}^{-1}$ under magic angle conditions and using a fast PMT detector (H10720) for fluorescence detection. Deconvolution of the data (4096 channels) was done by measuring the instrument response function with a scatterer (LUDOX).

Femtosecond Transient-Absorption Spectroscopy. The samples were dissolved in toluene (Uvasol from Merck), degassed for $30 \mathrm{~min}$, and filtered. The experiments were performed in $0.2 \mathrm{~mm}$ fused silica cuvettes (Spectrocell Inc.) at RT and the optical density was adjusted to ca. 0.3 at the corresponding excitation wavenumber. The pump-probe measurements were performed with a Helios transient spectrometer from Ultrafast Systems and a chirped pulse amplified femtosecond laser (Solstice) from Newport Spectra Physics (pulse length of $100 \mathrm{fs}$ ) with a repetition rate of $1 \mathrm{kHz}$ and a fundamental wavenumber of $12500 \mathrm{~cm}^{-1}(800 \mathrm{~nm})$. The output beam from the Solstice amplifier was split into two parts. A small part was focused onto a vertically oscillating $\mathrm{CaF}_{2}$ crystal to generate a white light continuum between $11900 \mathrm{~cm}^{-1}(840 \mathrm{~nm})$ and $25000 \mathrm{~cm}^{-1}(400 \mathrm{~nm})$, which was polarized horizontally and used as the probe pulse. The main part was used to pump a non-collinear optical parametric amplifier (NOPA) to generate the pump pulse with the corresponding excitation wavenumbers. By means of a wire grid (Moxtek) the polarization axis of the pump pulse was set to $54^{\circ}$ (magic angle) relative to that of the probe beam to avoid anisotropic effects. The pump pulse $(\varnothing \mathrm{ca} .0 .5 \mathrm{~mm})$ and probe pulse $(\varnothing \mathrm{ca} .0 .1 \mathrm{~mm})$ met at ca. $6^{\circ}$ vertical angle in the sample cuvette. The probe light was measured by a spectrograph equipped with a CMOS sensor (Ultrafast Systems, Helios) in the range between 11900 and $25000 \mathrm{~cm}^{-1}$ with an intrinsic resolution of $1.5 \mathrm{~nm}$. Every second pump pulse was blocked by a mechanical chopper (working at $500 \mathrm{~Hz}$ ) to measure $\mathrm{I}$ and $\mathrm{I}_{0}$. In order to compensate intensity fluctuations, a reference probe beam was split off and also detected with an identical spectrograph. By means of a computercontrolled linear stage (retro reflector in double pass setup) the relative temporal delay between pump and probe pulse was varied in $20 \mathrm{fs}$ steps from 0 fs to 4 ps and from 4 ps to $8 \mathrm{~ns}$ in logarithmic steps with a maximum step size of 200 ps. 
Steady state absorption spectra were recorded before and after the transient absorption experiment to exclude degradation of the sample. The raw data were corrected for stray light prior to data analysis of the difference spectra map (time $\times$ wavelength). The maps recorded under magic angle conditions were analyzed with GLOTARAN ${ }^{1,2}$ including the correction for the white light dispersion (chirp) and modelling the instrument response function and the coherent artefact.

Computational Aspects. Monte Carlo samplings for conformational analyses were carried out using the OPLS_2005 force field under solvent-free conditions applying the Maestro Molecular Modeling Interface from Schrödinger Inc. For dimeric species all conformers, except for enantiomeric counterparts, were submitted to further refinement with DFT. All further geometry optimizations and thermochemistry computations with the M052X functional were performed using Gaussian 09 software under Windows and UNIX OS, respectively. ${ }^{4}$ All geometry optimizations were run using the empirical dispersion correction (DFT-D3) of Grimme et al..$^{5}$ and the Polarizable Continuum Model (IEFPCM) for toluene under tight convergence criteria. Final single point refinements with the double-hybrid DSD-BLYP-D3 functional were performed with the SMD solvation model for toluene ${ }^{6}$ under tight convergence criteria and carried out with the program ORCA (version 4.0.0.2) from the working group of Prof. Frank Neese (Mühlheim an der Ruhr). ${ }^{7}$ 


\section{Spectroscopic Data of Prepared Final Compounds}

Table S1. Spectroscopic data of prepared compounds at room temperature.

\begin{tabular}{|c|c|c|c|c|c|c|c|c|}
\hline (oligo-)BODIPY & solvent & $\begin{array}{c}\lambda^{\mathrm{A}} \max \\
/ \mathrm{cm}^{-1}(\mathrm{~nm})^{a}\end{array}$ & $\begin{array}{c}\lambda_{\text {max }}^{\mathrm{F}} \\
/ \mathrm{cm}^{-1}(\mathrm{~nm})^{b}\end{array}$ & $\begin{array}{c}\Delta \tilde{v} \\
{\left[\mathrm{~cm}^{-1}\right]^{c}}\end{array}$ & $\begin{array}{c}\boldsymbol{E} \\
{\left[10^{3} \mathrm{M}^{-1} \mathrm{~cm}^{-1}\right]^{d}}\end{array}$ & $\begin{array}{c}\boldsymbol{\Phi}_{\mathbf{F}} \\
(\mathrm{RT})^{e}\end{array}$ & $\begin{array}{c}\tau_{\mathbf{F}} \\
{[\mathrm{ns}]^{f}}\end{array}$ & $\begin{array}{c}\boldsymbol{k}_{\mathrm{F}} \\
{\left[10^{8} \mathrm{~s}^{-1}\right]^{g}}\end{array}$ \\
\hline \multirow{3}{*}{ G (Monomer DM) } & DCM & $19880(503)$ & $19570(511)$ & 310 & 92 & 0.88 & 5.29 & 1.66 \\
\hline & THF & $19920(502)$ & $19610(510)$ & 310 & 96 & 0.98 & 5.23 & 1.87 \\
\hline & toluene & $19800(505)$ & $19490(513)$ & 310 & 98 & 0.88 & 4.63 & 1.90 \\
\hline \multirow{3}{*}{$\operatorname{Red}($ Monomer DM) } & DCM & $16560(604)$ & $14730(679)$ & 1830 & 89 & 0.69 & 3.55 & 1.94 \\
\hline & THF & $16610(602)$ & $14930(670)$ & 1680 & 96 & 0.61 & 3.36 & 1.82 \\
\hline & toluene & $16450(608)$ & $15630(640)$ & 820 & 100 & 0.83 & 3.41 & 2.43 \\
\hline \multirow{3}{*}{ 1G-Red } & DCM & $19920(502) / 16340(612)$ & $14680(681)$ & 1660 & $74 / 93$ & $0.03^{h}$ & $0.1^{h}$ & $2.7^{h}$ \\
\hline & THF & $19920(502) / 16390(610)$ & $14790(676)$ & 1600 & $75 / 102$ & $0.10^{h}$ & $0.4^{h}$ & $2.5^{h}$ \\
\hline & toluene & $19760(506) / 16230(616)$ & $15460(647)$ & 770 & $69 / 113$ & 0.85 & 3.33 & 2.56 \\
\hline \multirow{3}{*}{ 2G-Red } & DCM & $19310(518) / 16310(613)$ & $14730(679)$ & 1580 & $140 / 95$ & $0.01^{h}$ & $0.1^{h}$ & $0.7^{h}$ \\
\hline & THF & $19310(518) / 16390(610)$ & $14900(671)$ & 1490 & $152 / 105$ & $0.05^{h}$ & $0.3^{h}$ & $1.5^{h}$ \\
\hline & toluene & $19160(522) / 16210(617)$ & $15460(647)$ & 750 & $143 / 118$ & 0.82 & 3.16 & 2.59 \\
\hline \multirow{3}{*}{ 3G-Red } & DCM & $18980(527) / 16310(613)$ & $14710(680)$ & 1600 & $210 / 102$ & $0.02^{h}$ & $0.2^{h}$ & $0.1^{h}$ \\
\hline & THF & $18980(527) / 16390(610)$ & $14860(673)$ & 1530 & $244 / 115$ & $0.06^{h}$ & & i \\
\hline & toluene & $18830(531) / 16230(616)$ & $15460(647)$ & 770 & $228 / 125$ & 0.82 & 3.00 & 2.73 \\
\hline \multirow{3}{*}{ G| $\left.\right|^{\text {Et }}($ Monomer EDM $)$} & DCM & $19010(526)$ & $18620(537)$ & 390 & 70 & 0.94 & 6.01 & 1.56 \\
\hline & THF & $18980(527)$ & $18690(535)$ & 290 & 75 & 0.93 & 5.83 & 1.56 \\
\hline & toluene & $18900(529)$ & $18590(538)$ & 310 & 77 & 0.87 & 5.17 & 1.68 \\
\hline \multirow{3}{*}{$\left.\operatorname{Red}\right|^{\mathrm{Et}}($ Monomer EDM) } & DCM & $16260(615)$ & $14640(683)$ & 1620 & 75 & 0.71 & 3.76 & 1.88 \\
\hline & THF & $16260(615)$ & $14810(675)$ & 1450 & 80 & 0.67 & 3.59 & 1.87 \\
\hline & toluene & $16100(621)$ & $15290(654)$ & 810 & 86 & 0.80 & 3.72 & 2.15 \\
\hline \multirow{3}{*}{ 1G-Red $\left.\right|^{\text {Et }}$} & DCM & $18940(528) / 15970(626)$ & $14620(684)$ & 1350 & $49 / 84$ & $0.04^{h}$ & $0.3^{h}$ & 1.2 \\
\hline & THF & $18940(528) / 16000(625)$ & $14730(679)$ & 1270 & $48 / 80$ & 0.36 & 2.04 & 1.76 \\
\hline & toluene & $18870(530) / 15900(629)$ & $15220(657)$ & 680 & $48 / 91$ & 0.78 & 3.64 & 2.14 \\
\hline \multirow{3}{*}{ 2G-Red $\left.\right|^{\text {Et }}$} & DCM & $18280(547) / 16000(625)$ & $14660(682)$ & 1340 & $146 / 98$ & $0.05^{h}$ & $0.4^{h}$ & 1.4 \\
\hline & THF & $18320(546) / 16000(625)$ & $14680(681)$ & 1320 & $150 / 102$ & 0.29 & 2.25 & 1.29 \\
\hline & toluene & $18250(548) / 15870(630)$ & $15220(657)$ & 650 & $151 / 107$ & 0.83 & 3.47 & 2.39 \\
\hline \multirow{3}{*}{ 3G-Red $\left.\right|^{\mathrm{Et}}$} & DCM & $17920(558) / 16000(625)$ & $14680(681)$ & 1320 & $258 / 105$ & $0.06^{h}$ & $0.4^{h}$ & $1.6^{h}$ \\
\hline & THF & $17990(556) / 16050(623)$ & $14710(680)$ & 1340 & $267 / 110$ & 0.28 & $1.11 / 2.42^{j}$ & $2.52 / 1.16$ \\
\hline & toluene & $17860(560) / 15870(630)$ & $15220(657)$ & 650 & $259 / 111$ & 0.85 & 3.37 & 2.52 \\
\hline
\end{tabular}

${ }^{a}$ Energy of main BODIPY absorption bands. ${ }^{b}$ Energy of main emission band. ${ }^{c}$ Stokes shift referring to lowest energy absorption band. ${ }^{d}$ Extinction coefficient of main absorption bands. First value: high energy ( $g r e e n)$ band; second value, if noted, low energy $r e d$ band. ${ }^{\circ}$ Absolute fluorescence quantum yield. ${ }^{f}$ Experimental fluorescence lifetime (TCSPC). ${ }^{g}$ Fluorescence rate constant according to $\Phi_{\mathrm{F}} / \tau_{\mathrm{F}} .{ }^{h}$ Value with high uncertainty. ${ }^{i}$ Multiple lifetimes, maybe due to decomposition. ${ }^{j}$ Two lifetimes with a ratio $1 / 1$. 


\section{Building blocks, products and synthetic procedures}

3.1 Synthetic procedures for BODIPY monomers and DM-homooligomers.

\section{DM series}

The green building blocks G, 2G, 3G and 4G of the DM series were prepared using a literature-known procedure. $^{8}$

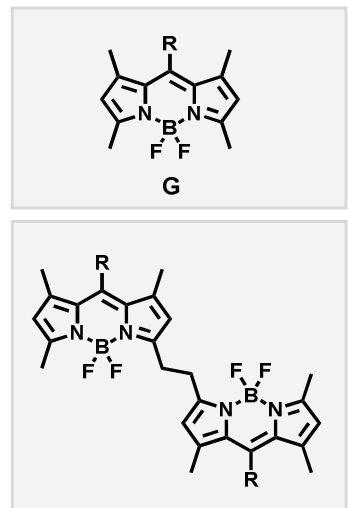

$2 \mathbf{G}$
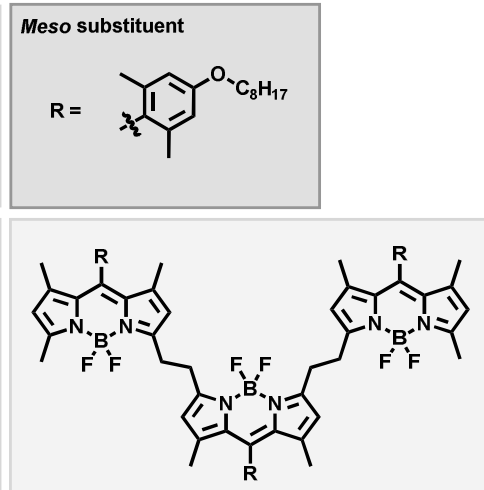

$3 G$

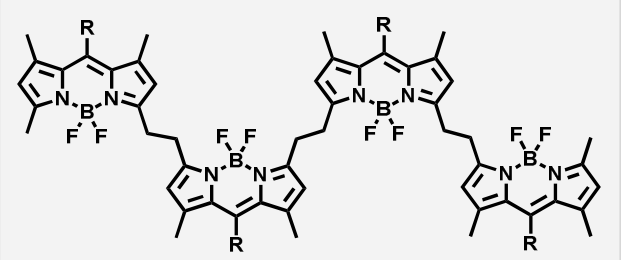

4G

\section{EDM series}

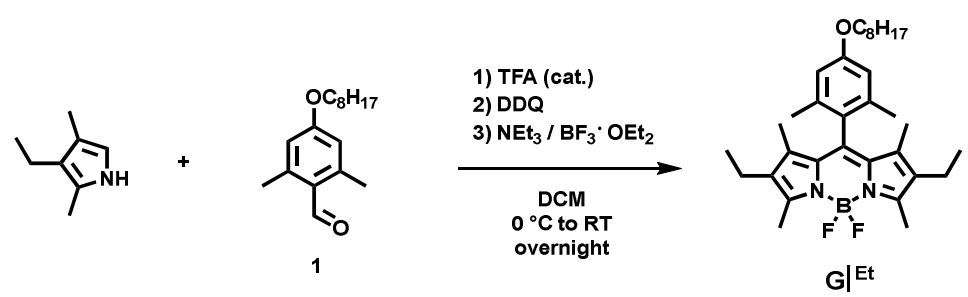

2-Ethyl-1,3-dimethylpyrrole ( $4.53 \mathrm{~mL}, 33.5 \mathrm{mmol}, 2.2$ equiv.) and the aromatic aldehyde $\mathbf{1}(4.00 \mathrm{~g}, 15.2 \mathrm{mmol}$, 1.0 equiv.) were dissolved in degassed dichloromethane $(750 \mathrm{~mL})$. Trifluoroacetic acid (TFA) $(58 \mu \mathrm{L}$, $0.76 \mathrm{mmol}, 0.05$ equiv.) were added and the reaction mixture stirred at room temperature overnight. Solid 2,3Dichloro-5,6-dicyano-1,4-benzoquinone (DDQ) (3.45 g, $15.2 \mathrm{mmol}, 1.0$ equiv.) was added and stirring continued at room temperature for $1 \mathrm{~h}$. Triethylamine $\left(\mathrm{NEt}_{3}\right)(21.0 \mathrm{~mL}, 152 \mathrm{mmol}, 10$ equiv. $)$ followed by $\mathrm{BF}_{3} \mathrm{OEt}_{2}\left(23 \mathrm{~mL}, 182 \mathrm{mmol}, 12\right.$ equiv.) were added at $0{ }^{\circ} \mathrm{C}$ and the reaction mixture warmed to $\mathrm{RT}$ for $2 \mathrm{~h}$ and afterwards quenched by addition of $\mathrm{NaHCO}_{3}$. The organic phase was thoroughly washed with saturated $\mathrm{NaHCO}_{3}$ solution (3 times) and finally with brine. The organic phase was dried with $\mathrm{Na}_{2} \mathrm{SO}_{4}$, the solvent removed under reduced pressure and the residue directly loaded onto the column. Column chromatography on silica gel (20\% DCM in pentane $\rightarrow 50 \%$ DCM in pentane) gave pure BODIPY monomer as an orange solid in $42 \%$ yield $(3.43 \mathrm{~g}, 6.38 \mathrm{mmol})$. 


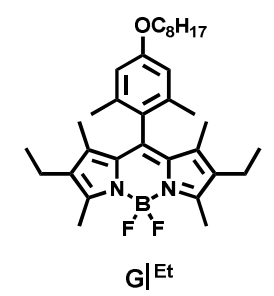

${ }^{1} \mathbf{H}$ NMR $\left(\mathrm{CDCl}_{3}, 300 \mathrm{MHz}\right): \delta(\mathrm{ppm}) 0.90(\mathrm{t}, J=6.8 \mathrm{~Hz}, 3 \mathrm{H}), 0.99(\mathrm{t}, J=7.5 \mathrm{~Hz}, 6 \mathrm{H}), 1.28-1.55(\mathrm{~m}, 10 \mathrm{H})$, $1.79(\mathrm{~m}, 2 \mathrm{H}), 2.09(\mathrm{~s}, 6 \mathrm{H}), 2.31(\mathrm{q}, J=7.5 \mathrm{~Hz}, 4 \mathrm{H}), 2.53(\mathrm{~s}, 6 \mathrm{H}), 3.97(\mathrm{t}, J=7.3 \mathrm{~Hz}, 2 \mathrm{H}), 6.68(\mathrm{~s}, 2 \mathrm{H})$.

${ }^{13}$ C NMR $\left(\mathrm{CDCl}_{3}, 75 \mathrm{MHz}\right): \delta$ (ppm) 10.80, 12.71, 14.30, 14.91, 17.28, 20.15, 22.86, 26.28, 29.44, 29.53, 29.61, 32.01, 68.10, 114.2, 127.3, 130.5, 132.5, 137.0, 137.8, 139.9, 153.5, 159.4.

${ }^{19} \mathbf{F ~ N M R}\left(\mathrm{CDCl}_{3}, 376 \mathrm{MHz}\right): \delta(\mathrm{ppm})-146.40\left(\mathrm{q}, J_{\mathrm{B}-\mathrm{F}}=33.5 \mathrm{~Hz}\right)$.

HR-MS (ESI): $m / z$ calcd for $\mathrm{C}_{33} \mathrm{H}_{47} \mathrm{BF}_{2} \mathrm{~N}_{2} \mathrm{ONa}^{+} 559.3642$, found 559.3647 .

IR (ATR): $\tilde{v}\left(\mathrm{~cm}^{-1}\right)$ 973, 1053, 1110, 1154, 1186, 1286, 1313, 1399, 1468, 1534, 1603, 2860, 2924.

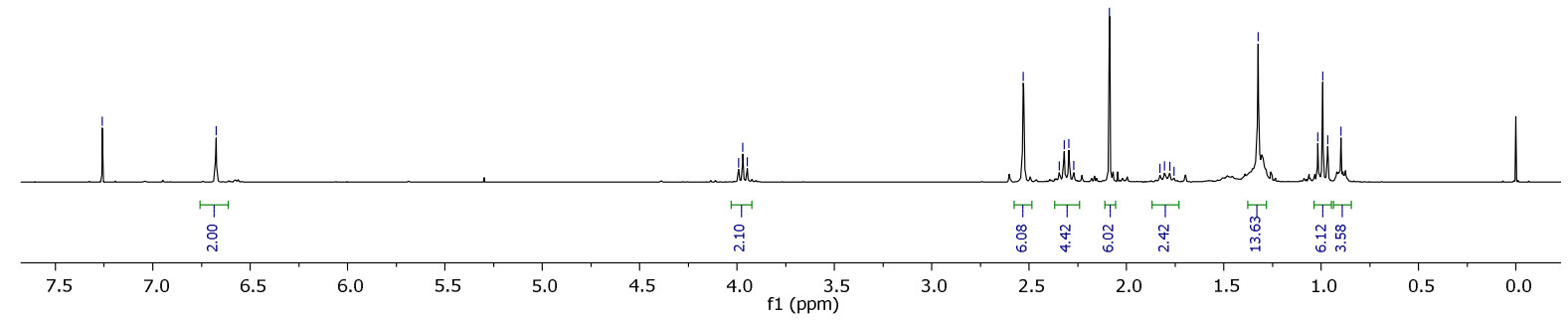

Figure S1. ${ }^{1} \mathrm{H}$ NMR $\left(\mathrm{CDCl}_{3}, 300 \mathrm{MHz}\right)$ spectrum of EDM monomer $\left.\mathbf{G}\right|^{\mathrm{Et}}$. 


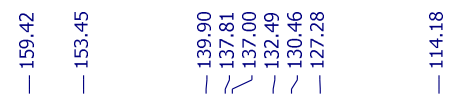
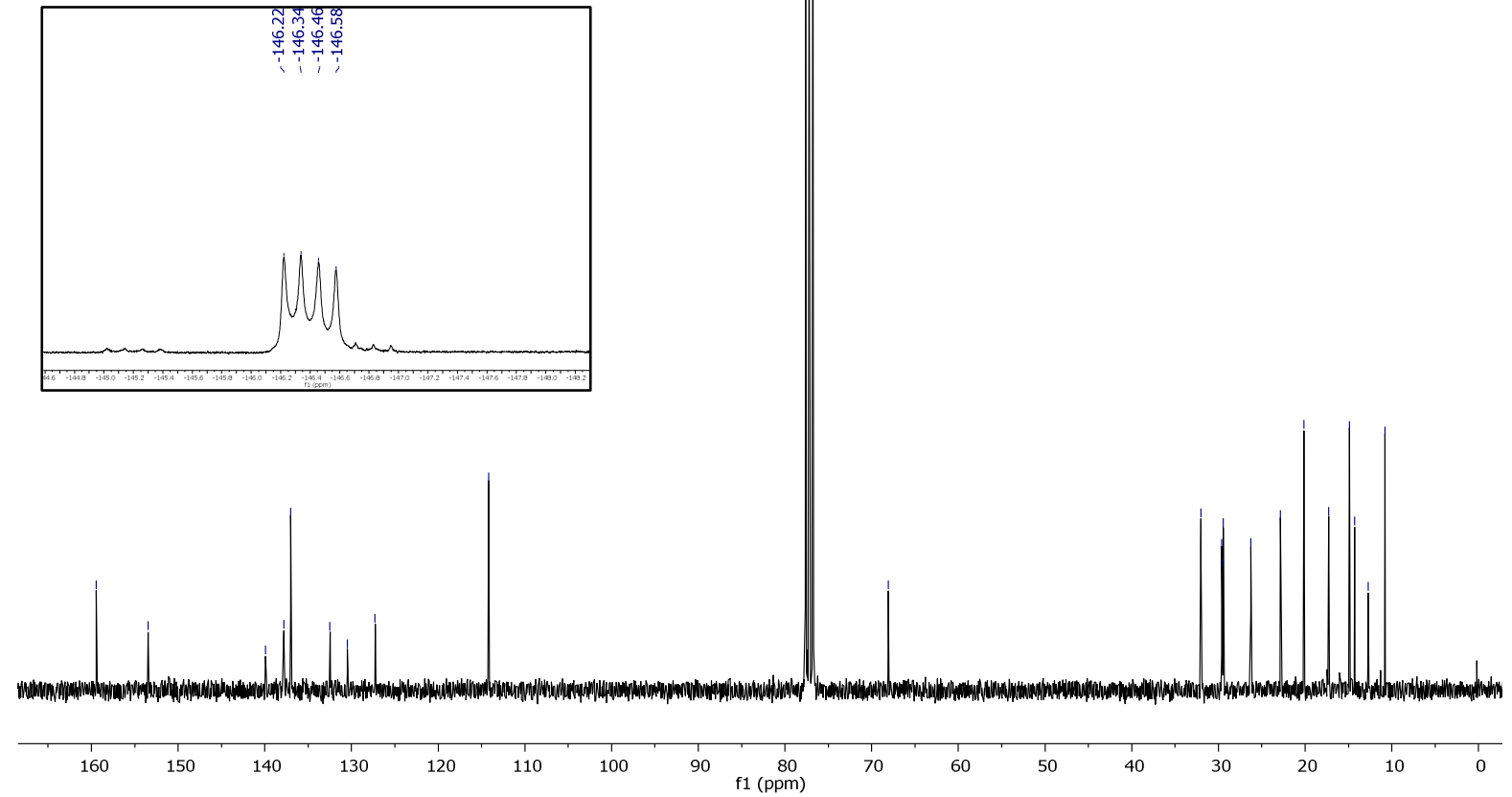

Figure S2. ${ }^{13} \mathrm{C} \mathrm{NMR}\left(\mathrm{CDCl}_{3}, 75 \mathrm{MHz}\right)$ spectrum of EDM monomer $\left.\mathbf{G}\right|^{\mathrm{Et}}$. 
3.2 Products obtained by hetero- and homo-oligomerizations and general synthetic procedures homodimerization

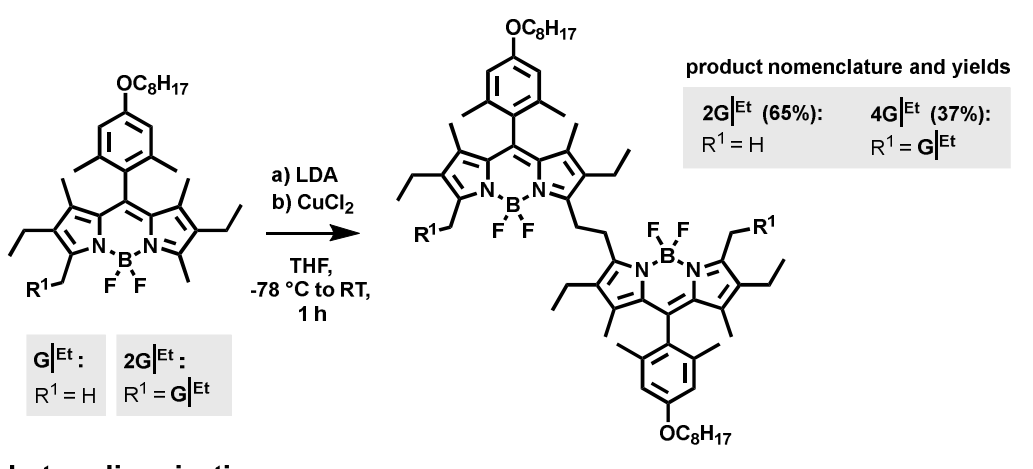

\section{heterodimerization}

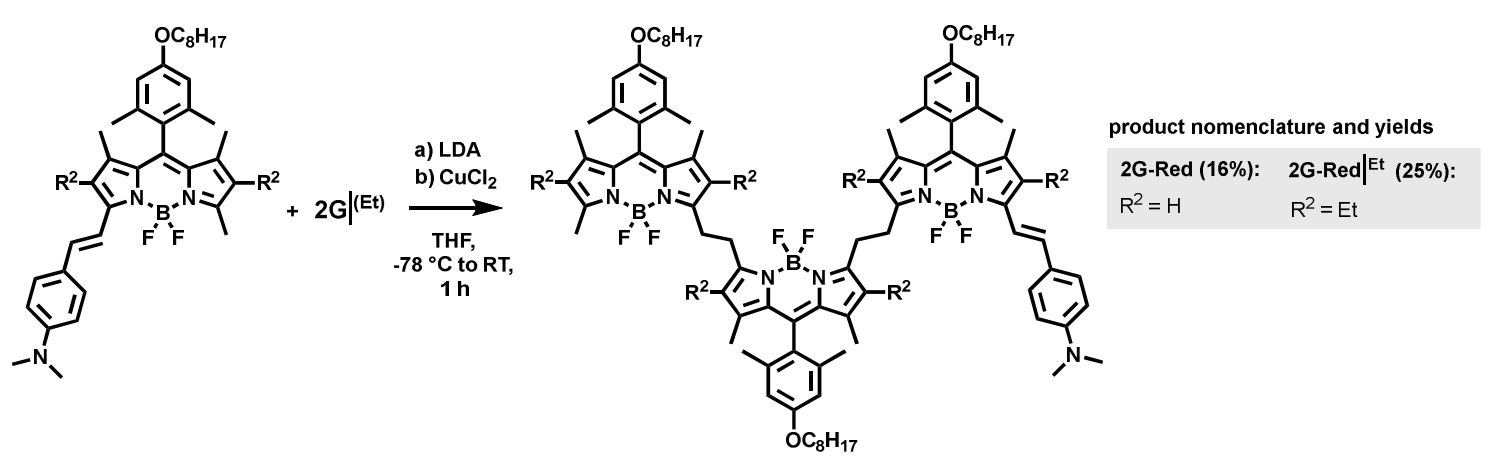

\section{General procedure for homodimerization}

The respective monomer $\left.\mathbf{G}\right|^{\mathrm{Et}}$ or dimer $\left.\mathbf{2 G}\right|^{\mathrm{Et}}(1.00 \mathrm{mmol}, 1.0$ equiv. $)$ was dissolved in THF $(10 \mathrm{~mL})$. At $-78^{\circ} \mathrm{C}$. LDA (1.0 M in THF) was added $(1.50 \mathrm{~mL}, 1.50 \mathrm{mmol}, 1.5$ equiv. $)$ and stirring continued for $30 \mathrm{~min}$ at $-78^{\circ} \mathrm{C}$. A solution of $\mathrm{CuCl}_{2}$ (carefully dried under high vacuum with heat gun) $(1.20 \mathrm{mmol}, 1.2$ equiv.) in DMF ( 1.2 $\mathrm{mL}$ ) was added dropwise and the reaction mixture allowed to warm to $0^{\circ} \mathrm{C}$ for ca. $10 \mathrm{~min}$ (depending on flask size) and afterwards quenched directly with aqueous saturated $\mathrm{NH}_{4} \mathrm{Cl}$ solution. After an aqueous workup with brine and dichloromethane, the organic layers were dried $\left(\mathrm{Na}_{2} \mathrm{SO}_{4}\right)$, solvents removed under reduced pressure and the residue directly submitted to column chromatography on silica gel in the case of a dimer formation or to gel permeation chromatography (JAIGEL-2H, chloroform) when dimers were subjected to the reaction procedure for oligomerization.

\section{General procedure for heterodimerization}

The respective styryl-equipped BODIPY Red|(Et) $(0.500 \mathrm{mmol}, 1.0$ equiv. $)$ and the corresponding dimer 2G| $\left.\right|^{(\mathrm{Et})}(0.500 \mathrm{mmol}, 1.0$ equiv. $)$ were dissolved in THF $(10 \mathrm{~mL})$. At $-78^{\circ} \mathrm{C} \mathrm{LDA}(1.0 \mathrm{M}$ in THF $)$ was added $\left(1.50 \mathrm{~mL}, 1.50 \mathrm{mmol}, 3.0\right.$ equiv.) and stirring continued for $30 \mathrm{~min}$ at $-78{ }^{\circ} \mathrm{C}$. A solution of $\mathrm{CuCl}_{2}$ (carefully dried under high vacuum with heat gun $)(1.25 \mathrm{mmol}, 2.5$ equiv. $)$ in DMF $(1.3 \mathrm{~mL})$ was added dropwise and the reaction mixture allowed to warm to $0{ }^{\circ} \mathrm{C}$ for ca. $10 \mathrm{~min}$ (depending on flask size) and afterwards quenched directly with aqueous saturated $\mathrm{NH}_{4} \mathrm{Cl}$ solution. After an aqueous workup with brine and dichloromethane, the organic layers were dried $\left(\mathrm{Na}_{2} \mathrm{SO}_{4}\right)$, solvents removed under reduced pressure and the residue directly submitted to gel permeation chromatography (JAIGEL-2H, chloroform) to separate the desired heteroproduct from correspondingly formed homodimers. The isolated heterotrimer 2G-Red $\left.\right|^{(\mathrm{Et})}$ was subsequently purified by measn of a short silica gel column to achieve a higher purity. 


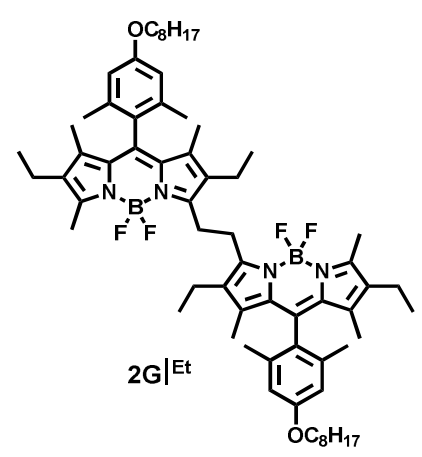

${ }^{1} \mathbf{H}$ NMR $\left(\mathrm{CDCl}_{3}, 300 \mathrm{MHz}\right): \delta(\mathrm{ppm}) 0.90(\mathrm{t}, J=6.7 \mathrm{~Hz}, 9 \mathrm{H}), 0.92-1.01(\mathrm{~m}, 18 \mathrm{H}), 1.20-1.55(\mathrm{~m}, 32 \mathrm{H})$, $1.81(\mathrm{~m}, 4 \mathrm{H}), 2.10(\mathrm{~s}, 12 \mathrm{H}), 2.32(\mathrm{q}, J=7.5 \mathrm{~Hz}, 4 \mathrm{H}), 2.42(\mathrm{q}, J=7.4 \mathrm{~Hz}, 4 \mathrm{H}), 2.55(\mathrm{~s}, 6 \mathrm{H}), 3.38(\mathrm{~s}, 4 \mathrm{H})$, $3.98(\mathrm{t}, J=7.3 \mathrm{~Hz}, 4 \mathrm{H}), 6.69(\mathrm{~s}, 4 \mathrm{H})$.

${ }^{13} \mathrm{C}$ NMR $\left(\mathrm{CDCl}_{3}, 75 \mathrm{MHz}\right): \delta(\mathrm{ppm}) 10.81,10.90,12.75,14.30,14.91,15.21,16.57,17.29,20.18,22.86$, $26.28,27.31,29.44,29.54,29.61,32.02,68.10,114.2,127.4,130.5,130.7,132.6,133.8,137.0,137.8,138.3$, $140.1,153.6,156.3,159.4$.

${ }^{19} \mathbf{F}$ NMR $\left(\mathrm{CDCl}_{3}, 376 \mathrm{MHz}\right): \delta(\mathrm{ppm})-143.61(\mathrm{~m}$,$) .$

HR-MS (ESI): $m / z$ calcd for $\mathrm{C}_{66} \mathrm{H}_{92} \mathrm{~B}_{2} \mathrm{~F}_{4} \mathrm{~N}_{4} \mathrm{O}_{2} \mathrm{Na}^{+} 1093.7235$, found 1093.7251 .

IR (ATR): $\tilde{v}\left(\mathrm{~cm}^{-1}\right)$ 967, 1003, 1053, 1112, 1142, 1179, 1202, 1317, 1385, 1410, 1446, 1473, 1531, 1802, 2115 , 2325, 2385, 2671, 2855, 2922.

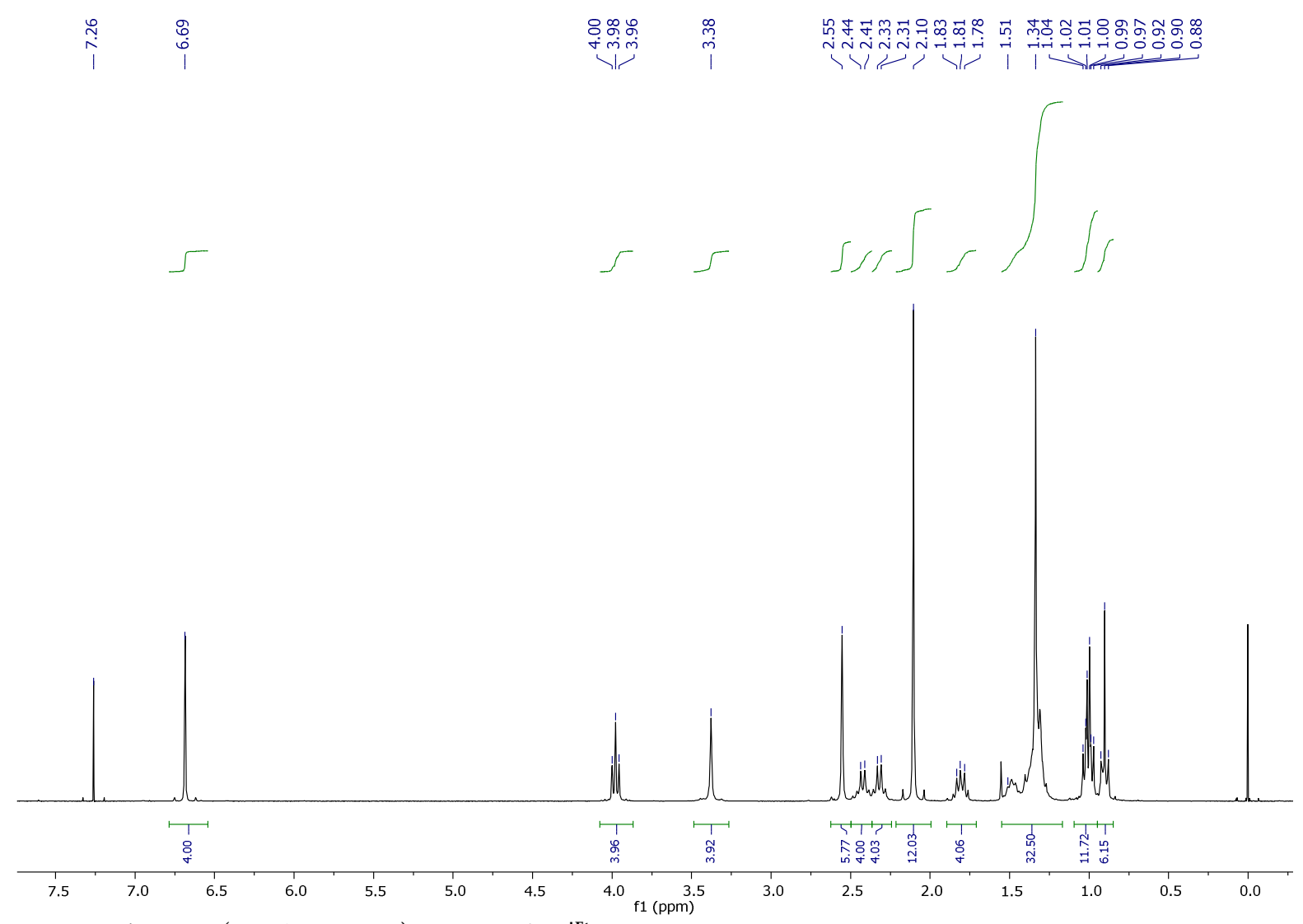

Figure S3. ${ }^{1} \mathrm{H} \mathrm{NMR}\left(\mathrm{CDCl}_{3}, 300 \mathrm{MHz}\right)$ spectrum of $\left.\mathbf{2 G}\right|^{\mathrm{Et}}$. 


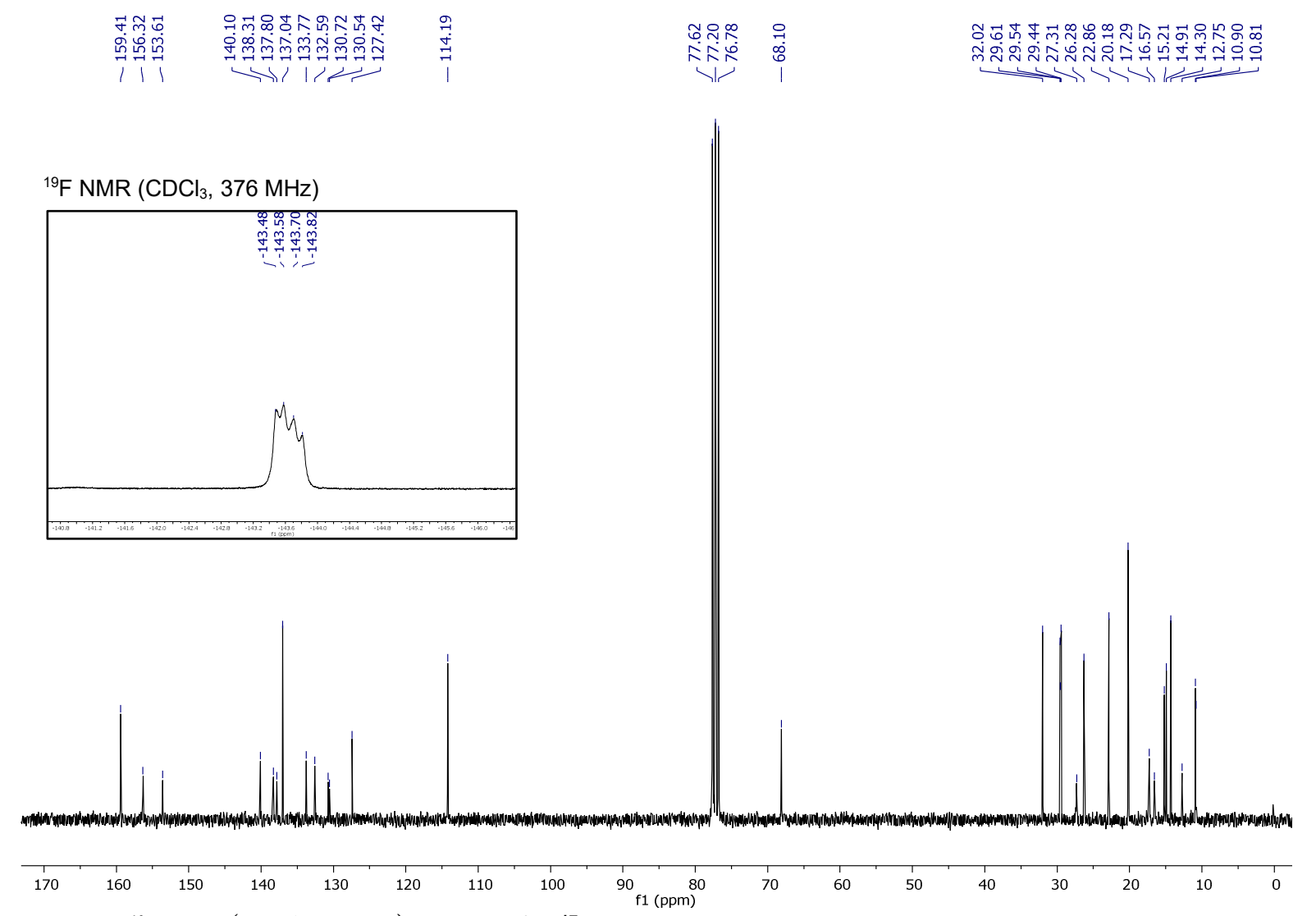

Figure S4. ${ }^{13} \mathrm{C} \mathrm{NMR}\left(\mathrm{CDCl}_{3}, 75 \mathrm{MHz}\right)$ spectrum of $\left.\mathbf{2 G}\right|^{\mathrm{Et}}$.

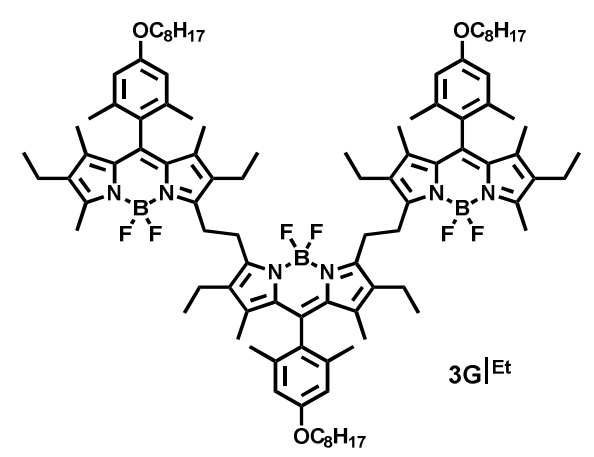

${ }^{1} \mathbf{H ~ N M R}\left(\mathrm{CDCl}_{3}, 300 \mathrm{MHz}\right): \delta(\mathrm{ppm}) 0.90(\mathrm{t}, J=6.8 \mathrm{~Hz}, 6 \mathrm{H}), 1.00(\mathrm{t}, J=7.5 \mathrm{~Hz}, 12 \mathrm{H}), 1.18-1.42(\mathrm{~m}, 42 \mathrm{H})$, $1.48(\mathrm{~m}, 6 \mathrm{H}), 1.55(\mathrm{~s}, 6 \mathrm{H}), 1.80(\mathrm{~m}, 6 \mathrm{H}), 2.10(\mathrm{~s}, 12 \mathrm{H}), 2.11(\mathrm{~s}, 6 \mathrm{H}), 2.32(\mathrm{~m}, 12 \mathrm{H}), 2.55(\mathrm{~s}, 6 \mathrm{H}), 3.42(\mathrm{~s}$, $8 \mathrm{H}), 3.98(\mathrm{~m}, 6 \mathrm{H}), 6.68(\mathrm{~s}, 4 \mathrm{H}), 6.69(\mathrm{~s}, 2 \mathrm{H})$.

${ }^{13} \mathrm{C}$ NMR $\left(\mathrm{CDCl}_{3}, 75 \mathrm{MHz}\right): \delta$ (ppm) 10.82, 10.90, 10.94, 12.77, 14.31, 14.92, 15.14, 15.19, 16.52, 16.60, $17.30,20.16,20.17,22.87,26.28,27.25,29.44,29.54,29.61,32.02$, 68.10, 114.2, 127.4, 127.6, 130.6, 130.7, $130.9,132.6,133.9,134.0,137.0,137.8,138.2,138.3,140.1,140.3,153.6,156.4,156.6,159.4$.

${ }^{19} \mathrm{~F} \mathrm{NMR}\left(\mathrm{CDCl}_{3}, 376 \mathrm{MHz}\right): \delta(\mathrm{ppm})-141.21$ (m, $\left.2 \mathrm{~F}\right),-143.61$ (m, $\left.4 \mathrm{~F}\right)$.

HR-MS (ESI): $m / z$ calcd for $\mathrm{C}_{99} \mathrm{H}_{137} \mathrm{~B}_{3} \mathrm{~F}_{6} \mathrm{~N}_{6} \mathrm{O}_{3} \mathrm{Na}^{+} 1628.0828$, found 1628.0864 .

IR (ATR): $\tilde{v}\left(\mathrm{~cm}^{-1}\right)$ 932, 969, 1011, 1044, 1125, 1179, 1201, 1267, 1316, 1411, 1447, 1470, 1532, 1605, 1764, 2115, 2326, 2385, 2670, 2858, 2924. 


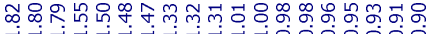

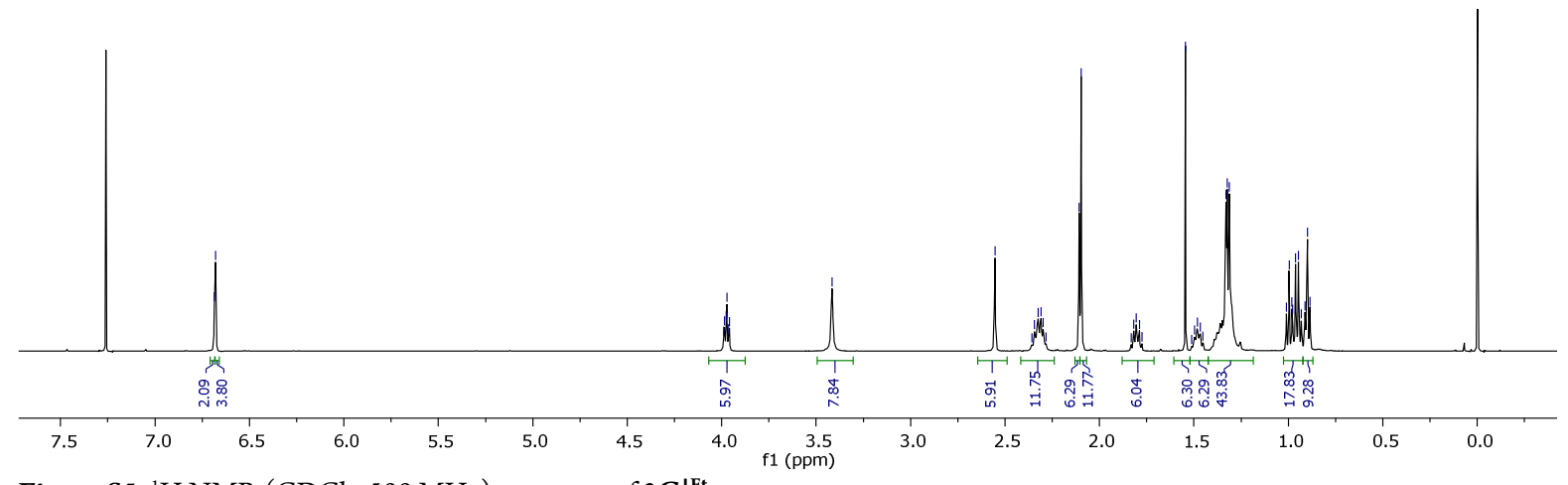

Figure S5. ${ }^{1} \mathrm{H}$ NMR $\left(\mathrm{CDCl}_{3}, 500 \mathrm{MHz}\right)$ spectrum of $\left.3 \mathbf{G}\right|^{\mathrm{Et}}$.

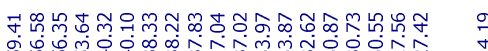

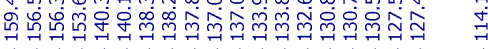

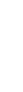

${ }^{19} \mathrm{~F} \mathrm{NMR}\left(\mathrm{CDCl}_{3}, 376 \mathrm{MHz}\right)$
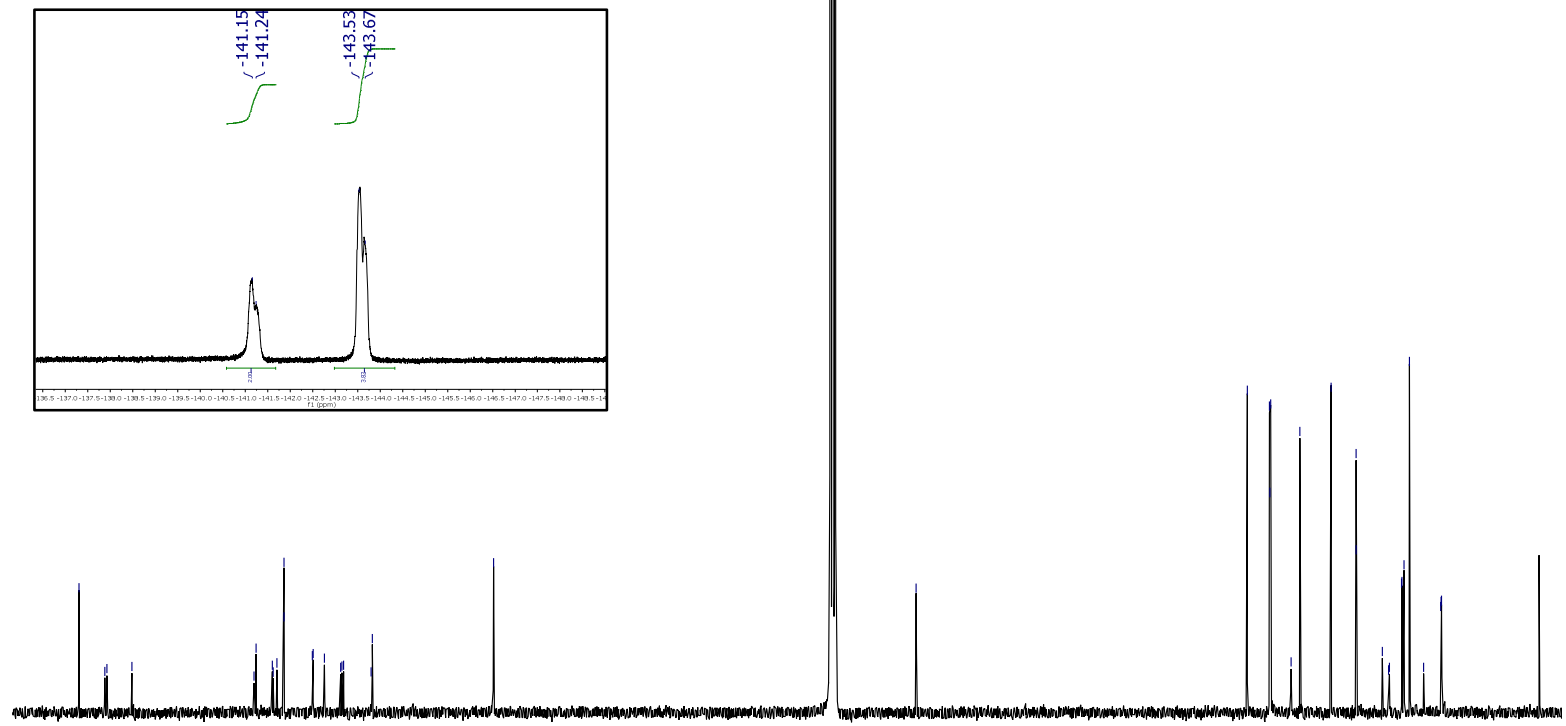

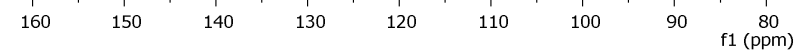

Figure S6. ${ }^{13} \mathrm{C} \mathrm{NMR}\left(\mathrm{CDCl}_{3}, 5 \mathrm{MHz}\right)$ spectrum of $\left.3 \mathbf{G}\right|^{\mathrm{Et}}$. 


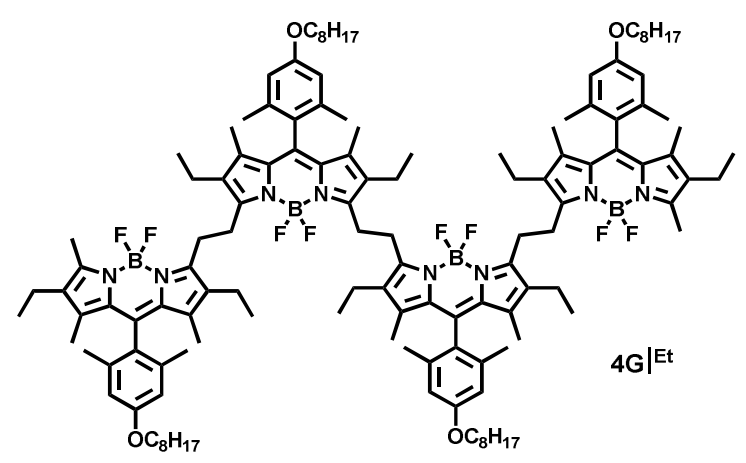

${ }^{1} \mathbf{H}$ NMR $\left(\mathrm{CDCl}_{3}, 500 \mathrm{MHz}\right): \delta(\mathrm{ppm}) 0.86-1.04(\mathrm{~m}, 36 \mathrm{H}), 1.20-1.42(\mathrm{~m}, 58 \mathrm{H}), 1.48(\mathrm{~m}, 8 \mathrm{H}), 1.55(\mathrm{~s}$, $8 \mathrm{H}), 1.80(\mathrm{~m}, 8 \mathrm{H}), 2.10(\mathrm{~s}, 12 \mathrm{H}), 2.11(\mathrm{~s}, 12 \mathrm{H}), 2.20-2.40(\mathrm{~m}, 16 \mathrm{H}), 2.55(\mathrm{~s}, 6 \mathrm{H}), 3.43(\mathrm{~m}, 12 \mathrm{H}), 3.98$ $(\mathrm{m}, 8 \mathrm{H}), 6.68(\mathrm{~s}, 4 \mathrm{H}), 6.69(\mathrm{~s}, 4 \mathrm{H})$.

${ }^{13} \mathrm{C}$ NMR $\left(\mathrm{CDCl}_{3}, 75 \mathrm{MHz}\right): \delta(\mathrm{ppm})$ 10.82, 10.90, 10.93, 12.77, 14.31, 14.92, 15.13, 15.16, 16.57, 17.30, 20.15, 20.16, 22.87, 26.28, 27.21, 29.44, 29.54, 29.61, 32.02, 68.10, 114.2, 127.4, 127.6, 130.6, 130.7, 130.9, 132.6, 133.9, 134.0, 134.1, 137.0, 137.8, 138.2, 138.3, 140.1, 140.3, 153.7, 156.4, 156.6, 156.7, 159.4.

${ }^{19} \mathbf{F ~ N M R}\left(\mathrm{CDCl}_{3}, 376 \mathrm{MHz}\right): \delta$ (ppm) -141.02 (m, $\left.4 \mathrm{~F}\right),-143.63$ (m, $\left.4 \mathrm{~F}\right)$.

HR-MS (ESI): $m / z$ calcd for $\mathrm{C}_{132} \mathrm{H}_{182} \mathrm{~B}_{4} \mathrm{~F}_{8} \mathrm{~N}_{8} \mathrm{O}_{4} \mathrm{Na}^{+} 2162.4421$, found 2162.4455 .

IR (ATR): $\tilde{v}\left(\mathrm{~cm}^{-1}\right)$ 933, 969, 1016, 1043, 1122, 1179, 1202, 1268, 1317, 1411, 1446, 1532, 2861, 2925.

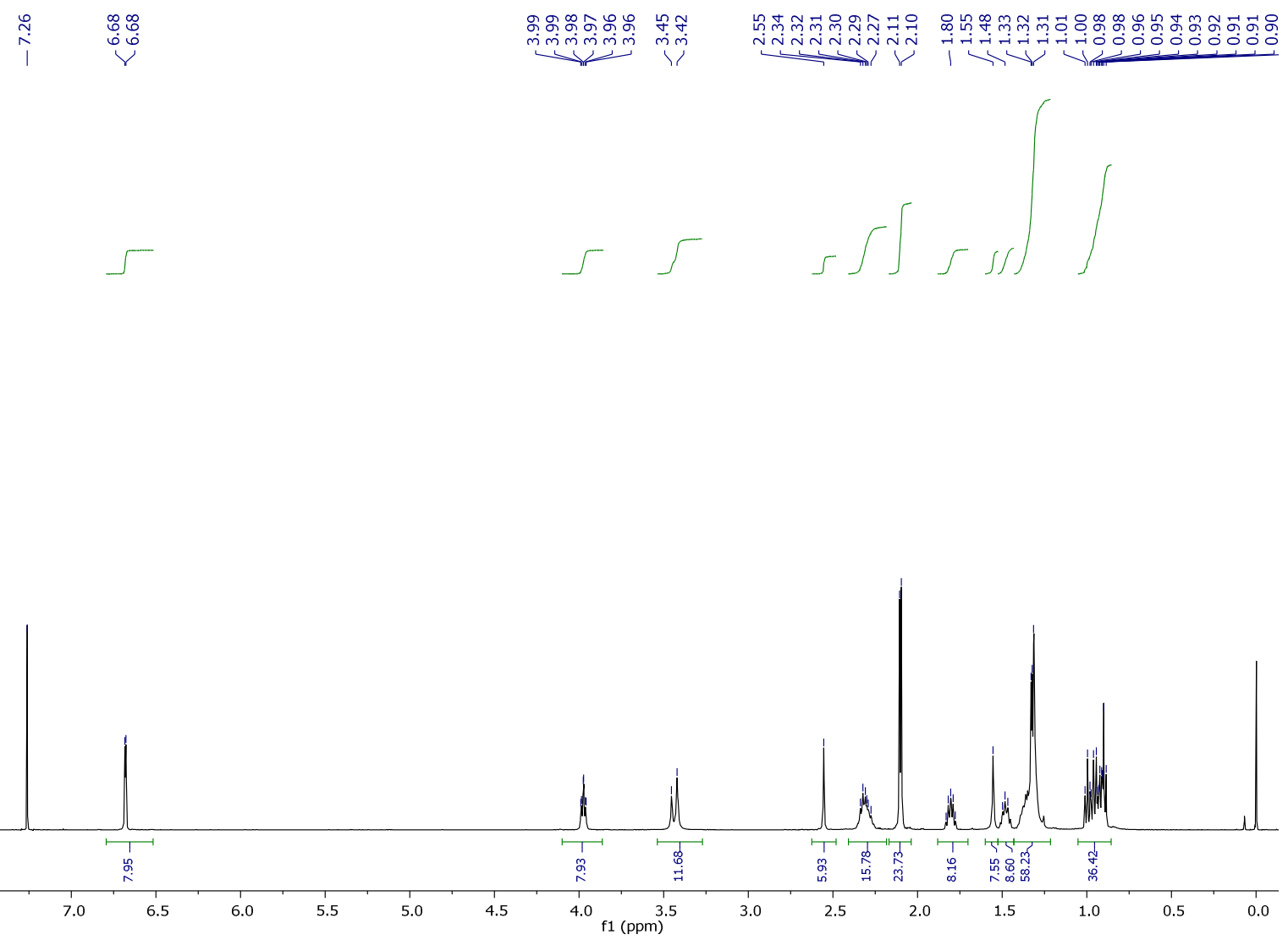

Figure S7. ${ }^{1} \mathrm{H} \mathrm{NMR}\left(\mathrm{CDCl}_{3}, 500 \mathrm{MHz}\right)$ spectrum of $\left.4 \mathbf{G}\right|^{\mathrm{Et}}$. 


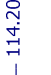

${ }^{19} \mathrm{~F} \mathrm{NMR}\left(\mathrm{CDCl}_{3}, 376 \mathrm{MHz}\right)$
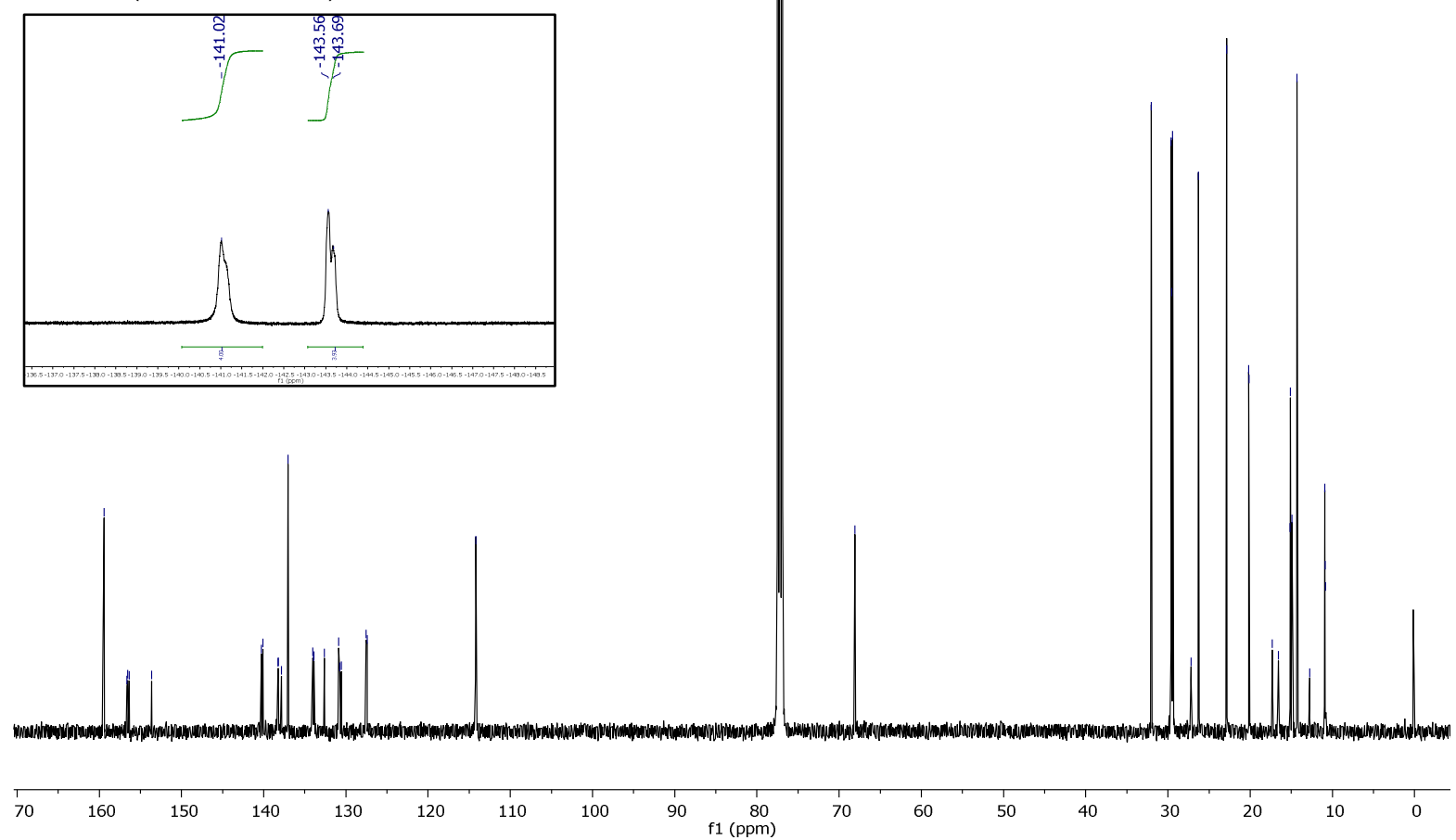

Figure S8. ${ }^{13} \mathrm{C} \mathrm{NMR}\left(\mathrm{CDCl}_{3}, 125 \mathrm{MHz}\right)$ spectrum of $\left.4 \mathbf{G}\right|^{\mathrm{Et}}$.

3.3 Absorption and fluorescence spectra of homooligomers $\left.\mathbf{2 G}\right|^{\mathrm{Et}},\left.\mathbf{3 G}\right|^{\mathrm{Et}}$ and $\left.\mathbf{4 G}\right|^{\mathrm{Et}}$

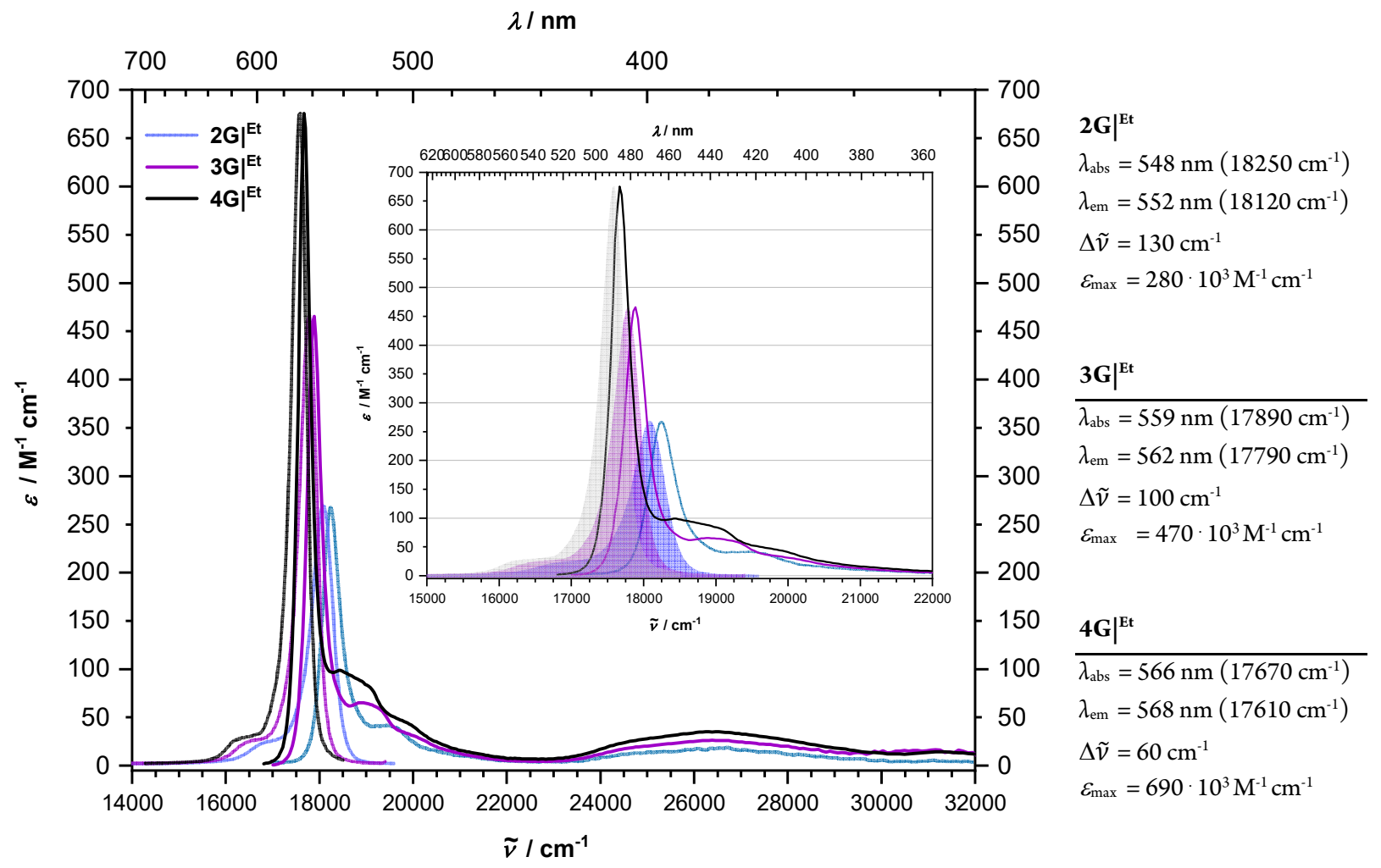

Figure S9. Absorption and emission spectra of $\left.\mathbf{2 G}\right|^{\mathrm{Et}},\left.\mathbf{3 G}\right|^{\mathrm{Et}}$ and $\left.4 \mathbf{G}\right|^{\mathrm{Et}}$ in toluene at room temperature. Emission is normalized. 


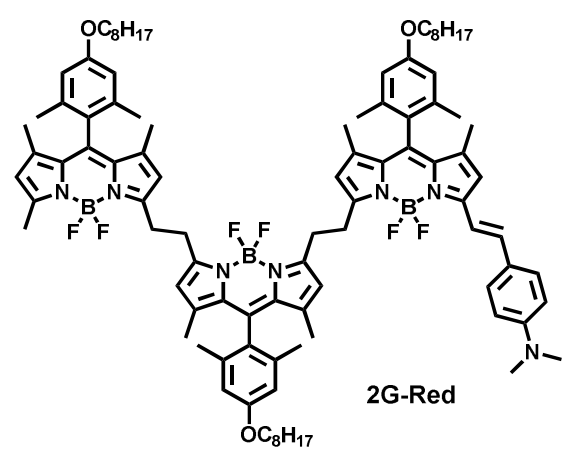

${ }^{1} \mathbf{H}$ NMR $\left(\mathrm{CDCl}_{3}, 300 \mathrm{MHz}\right): \delta(\mathrm{ppm}) 0.91(\mathrm{~m}, 9 \mathrm{H}), 1.26-1.52(\mathrm{~m}, 48 \mathrm{H}), 1.80(\mathrm{~m}, 6 \mathrm{H}), 2.08-2.13(\mathrm{~s}, 18$ H), $2.55(\mathrm{~s}, 3 \mathrm{H}), 3.01(\mathrm{~s}, 6 \mathrm{H}), 3.41(\mathrm{~s}, 4 \mathrm{H}), 3.45(\mathrm{~s}, 4 \mathrm{H}), 3.96(\mathrm{~m}, 6 \mathrm{H}), 5.96(\mathrm{~s}, 1 \mathrm{H}), 6.14(\mathrm{~s}, 1 \mathrm{H}), 6.17(\mathrm{~s}$, $2 \mathrm{H}), 6.21(\mathrm{~s}, 1 \mathrm{H}), 6.58(\mathrm{~s}, 1 \mathrm{H}), 6.68(\mathrm{~m}, 8 \mathrm{H}), 7.20(\mathrm{~d}, J=16.0 \mathrm{~Hz}, 1 \mathrm{H}), 7.46-7.54(\mathrm{~m}, 3 \mathrm{H})$.

${ }^{13} \mathbf{C}$ NMR $\left(\mathrm{CDCl}_{3}, 75 \mathrm{MHz}\right): \delta(\mathrm{ppm}) 13.69,13.70,13.80,14.30,20.07,20.12,20.17,22.86,26.28,29.43$, 29.54, 29.61, 32.01, 40.43, 68.09, 112.2, 114.2, 114.3, 119.9, 125.0, 125.1, 126.5, 126.6, 126.9, 129.4, 131.1, 131.3, 136.76, 136.81, 137.2, 141.9, 142.2, 142.5, 142.7, 151.2, 155.5, 155.8, 158.1, 158.2, 159.5, 159.6 .

${ }^{19} \mathbf{F ~ N M R}\left(\mathrm{CDCl}_{3}, 376 \mathrm{MHz}\right): \delta(\mathrm{ppm})-141.85$ (m, $\left.2 \mathrm{~F}\right),-144.33$ (m, $\left.2 \mathrm{~F}\right),-145.59$ (m, $\left.2 \mathrm{~F}\right)$.

HR-MS (ESI): $m / z$ calcd for $\mathrm{C}_{96} \mathrm{H}_{122} \mathrm{~B}_{3} \mathrm{~F}_{6} \mathrm{~N}_{7} \mathrm{O}_{3} \mathrm{Na}^{+} 1590.9685$, found 1590.9720 .

IR (ATR): $\tilde{v}\left(\mathrm{~cm}^{-1}\right)$ 700, 810, 977, 1030, 1074, 1104, 1150, 1180, 1209, 1304, 1362, 1447, 1497, 1538, 1594, $2856,2923$.

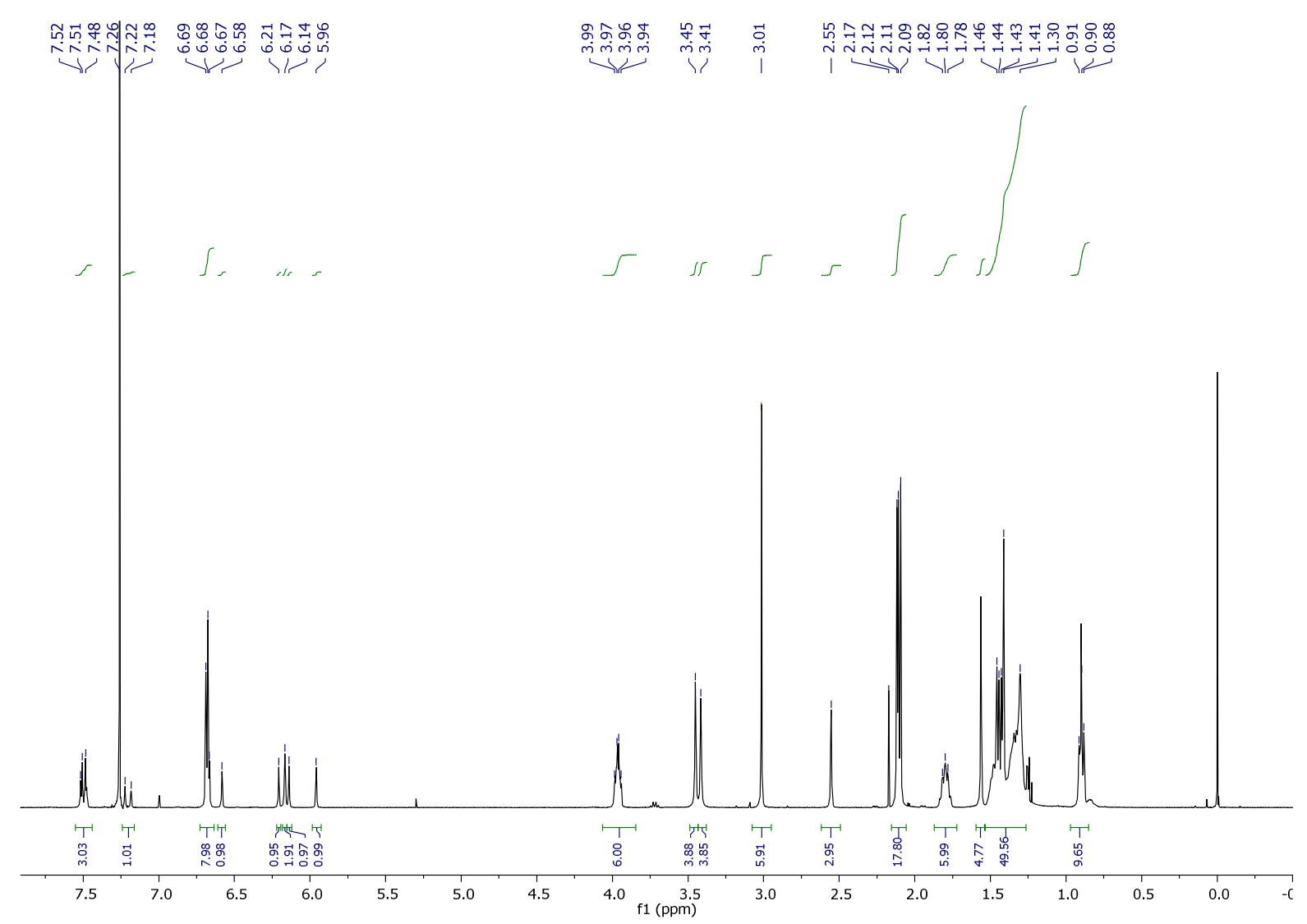

Figure S10. ${ }^{1} \mathrm{H} \mathrm{NMR}\left(\mathrm{CDCl}_{3}, 400 \mathrm{MHz}\right)$ spectrum of $\mathbf{2 G - R e d}$. 

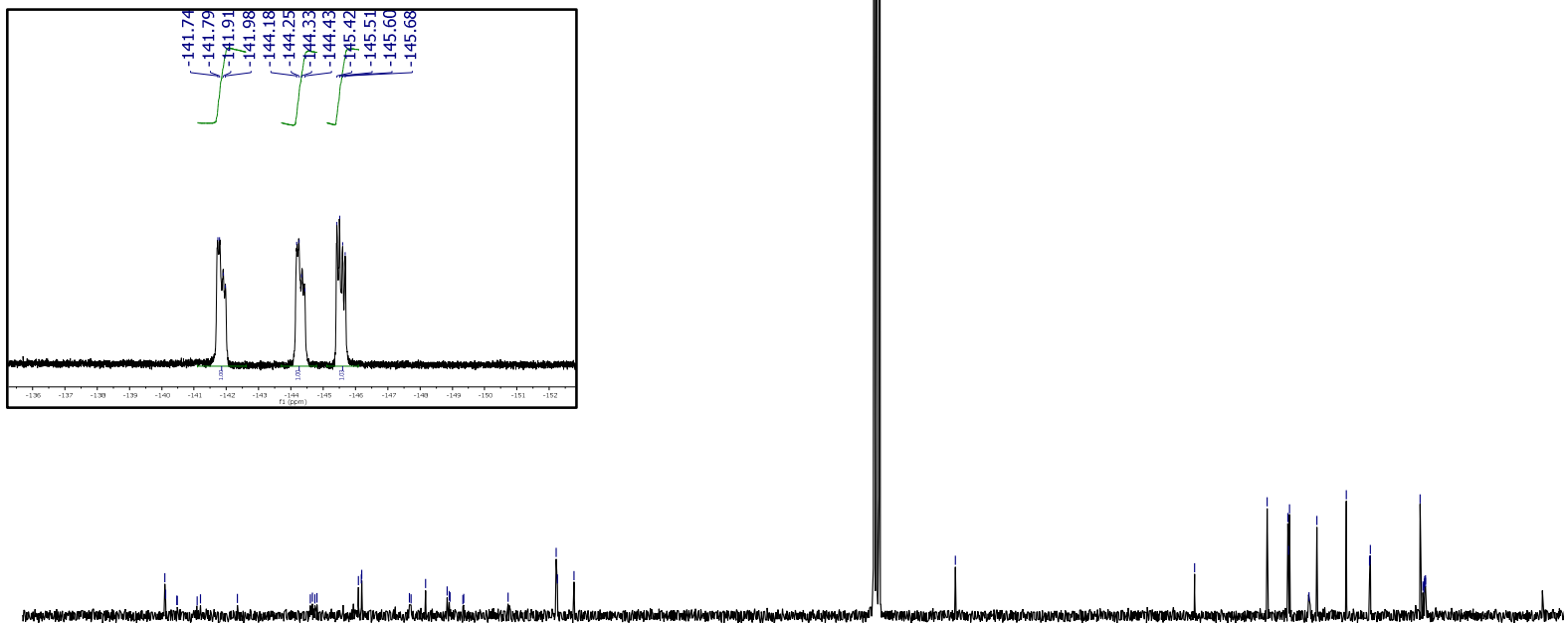

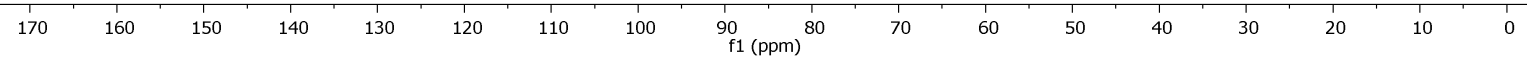

Figure S1 1. ${ }^{13} \mathrm{C} \mathrm{NMR}\left(\mathrm{CDCl}_{3}, 100 \mathrm{MHz}\right)$ spectrum of 2G-Red.

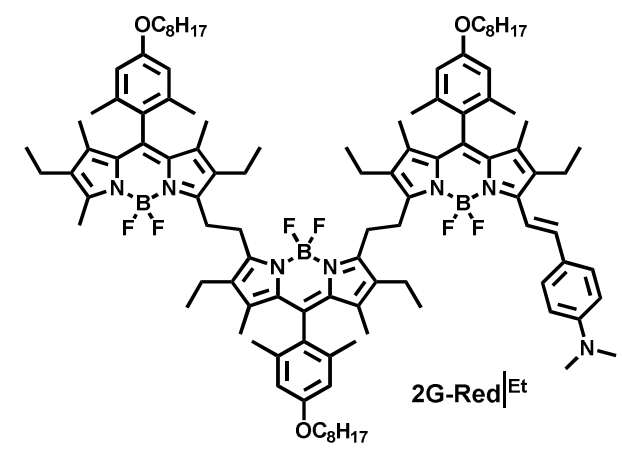

${ }^{1}$ H NMR $\left(\mathrm{CDCl}_{3}, 300 \mathrm{MHz}\right): \delta(\mathrm{ppm}) 0.85-1.10(\mathrm{~m}, 24 \mathrm{H}), 1.15(\mathrm{t}, J=7.4 \mathrm{~Hz}, 3 \mathrm{H}), 1.25-1.45(\mathrm{~m}, 42 \mathrm{H})$, $1.45-1.55(\mathrm{~m}, 6 \mathrm{H}), 1.82(\mathrm{~m}, 6 \mathrm{H}), 2.08-2.18(\mathrm{~s}, 18 \mathrm{H}), 2.25-2.50(\mathrm{~m}, 10 \mathrm{H}), 2.56(\mathrm{~s}, 3 \mathrm{H}), 2.63\left(\mathrm{~s}_{\mathrm{br}}, 2 \mathrm{H}\right)$, $3.05\left(\mathrm{~s}_{\mathrm{br}}, 6 \mathrm{H}\right), 3.43(\mathrm{~m}, 8 \mathrm{H}), 3.99(\mathrm{~m}, 6 \mathrm{H}) 6.69(\mathrm{~m}, 8 \mathrm{H}), 7.23(\mathrm{~d}, J=16.4 \mathrm{~Hz}, 1 \mathrm{H}), 7.51(\mathrm{~m}, 2 \mathrm{H}), 7.63\left(\mathrm{~s}_{\mathrm{br}}\right.$, $1 \mathrm{H})$.

${ }^{13} \mathrm{C}$ NMR $\left(\mathrm{CDCl}_{3}, 75 \mathrm{MHz}\right): \delta$ (ppm) 10.52, 10.81, 10.89, 10.92, 11.01, 12.77, 14.31, 14.92, 15.12, 15.19, $15.30,16.51,16.62,17.29,20.16,20.18,20.27,22.87,26.28,27.23,29.44,29.54,29.61,32.02,112.4,114.1$, 114.2 , 127.4, 127.6, 127.7, 128.7, 130.5, 130.7, 130.8, 130.9, 132.6, 133.89, 133.91, 134.0, 137.0, 137.1, 137.3, $137.8,138.17,138.20,138.44,140.1,140.2,150.6,153.6,156.37,156.43,156.8,159.4$.

${ }^{19}$ F NMR $\left(\mathrm{CDCl}_{3}, 376 \mathrm{MHz}\right): \delta$ (ppm) -140.25 (m, $\left.2 \mathrm{~F}\right),-141.18$ (m, $\left.2 \mathrm{~F}\right),-143.60$ (m, $\left.2 \mathrm{~F}\right)$.

HR-MS (ESI): $m / z$ calcd for $\mathrm{C}_{108} \mathrm{H}_{146} \mathrm{~B}_{3} \mathrm{~F}_{6} \mathrm{~N}_{7} \mathrm{O}_{3} \mathrm{Na}^{+} 1759.1563$, found 1759.1592 .

IR (ATR): $\tilde{v}\left(\mathrm{~cm}^{-1}\right) 700,766,808,855,933,963,1040,1125,1176,1203,1266,1315,1362,1384,1410$, 1442, 1527, 1600, 1795, 2118, 2325, 2385, 2856, 2924. 


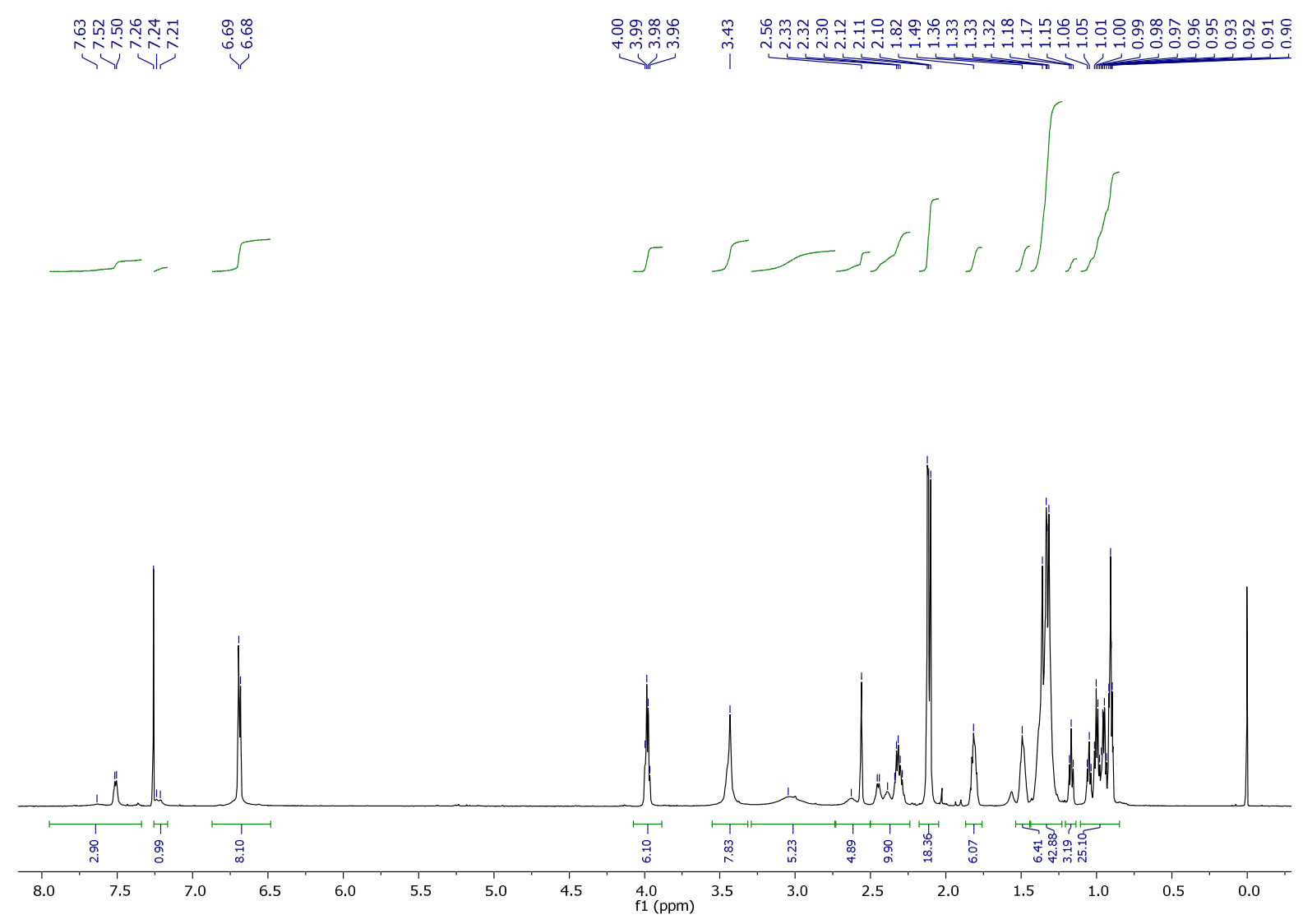

Figure S12. ${ }^{1} \mathrm{H} \mathrm{NMR}\left(\mathrm{CDCl}_{3}, 600 \mathrm{MHz}\right)$ spectrum of $\left.\mathbf{2 G - R e d}\right|^{\mathrm{Et}}$.

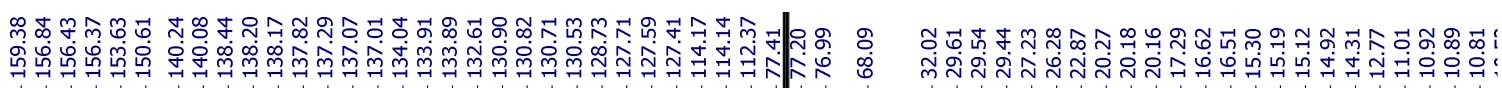

${ }^{19} \mathrm{~F} \mathrm{NMR}\left(\mathrm{CDCl}_{3}, 376 \mathrm{MHz}\right)$
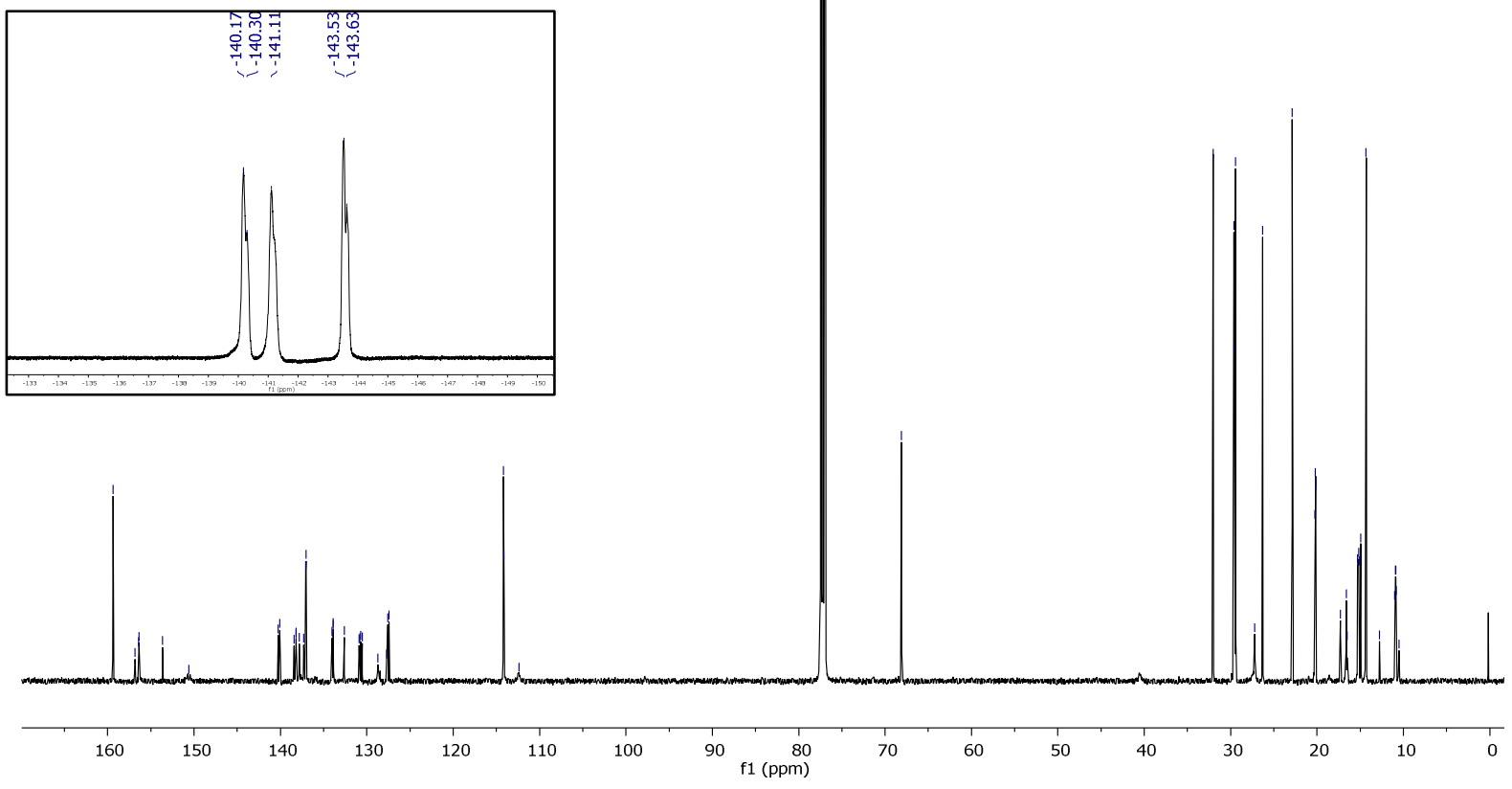

Figure S13. ${ }^{13} \mathrm{C} \mathrm{NMR}\left(\mathrm{CDCl}_{3}, 150 \mathrm{MHz}\right)$ spectrum of $\left.\mathbf{2 G - R e d}\right|^{\mathrm{Et}}$. 
3.4 Products obtained by Knoevenagel condensations and general synthetic procedure

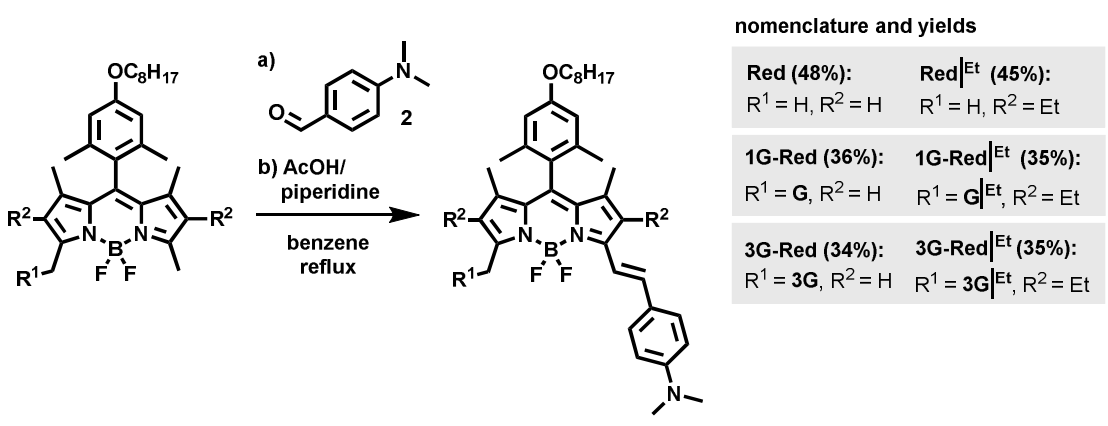

The respective monomer $\left.\mathbf{G}\right|^{(\mathrm{Et})}$, dimer $\left.\mathbf{2 G}\right|^{(\mathrm{Et})}$ and trimer $\left.\mathbf{3 G}\right|^{(\mathrm{Et})}(0.100 \mathrm{mmol}, 1.0$ equiv. $)$ was dissolved in benzene $(10 \mathrm{~mL})$. Aldehyde $2(0.600 \mathrm{mmol}, 6.0$ equiv.) was added followed by acetic acid ( $4.00 \mathrm{mmol}, 40$ equiv.) and piperidine ( $4.00 \mathrm{mmol}, 40$ equiv.). The reaction flask was equipped with a self-made reflux condenser filled with activated molecular sieves $(4 \AA)$ and the reaction mixture refluxed for several hours. The reaction time strongly depends on the activity and amount of the employed molecular sieves and the dryness of solvents and reagents. The reaction progress was carefully monitored by TLC and the reaction mixture cooled down to RT when the ratio of starting material and products was regarded as satisfactory. After an aqueous workup with brine and EtOAc, the organic layers were dried $\left(\mathrm{Na}_{2} \mathrm{SO}_{4}\right)$, solvents removed under reduced pressure and the residue put onto a silica gel column. With pure DCM as eluent both products and the starting material were isolated first. The product mixture was further submitted to gel permeation chromatography (JAIGEL-2H, chloroform) giving the respective monosubstituted styryl-equipped product in a typical yield range of $30-50 \%$ together with small amounts of the disubstituted congener and reisolated starting material.

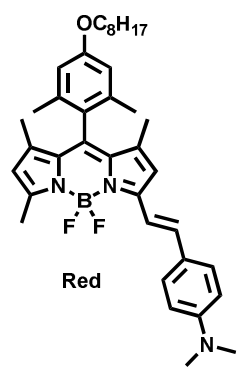

${ }^{1} \mathbf{H}$ NMR $\left(\mathrm{CDCl}_{3}, 300 \mathrm{MHz}\right): \delta(\mathrm{ppm}) 0.92(\mathrm{t}, J=7.4 \mathrm{~Hz}, 3 \mathrm{H}), 1.20-1.60(\mathrm{~m}, 16 \mathrm{H}), 1.82(\mathrm{~m}, 2 \mathrm{H}), 2.13(\mathrm{~s}$, $6 \mathrm{H}), 2.61(\mathrm{~s}, 3 \mathrm{H}), 3.02(\mathrm{~s}, 6 \mathrm{H}), 3.98(\mathrm{t}, J=6.5 \mathrm{~Hz}, 2 \mathrm{H}), 5.97(\mathrm{~s}, 1 \mathrm{H}), 6.60(\mathrm{~s}, 1 \mathrm{H}), 6.69(\mathrm{~m}, 4 \mathrm{H}), 7.23(\mathrm{~d}, J$ $=16.2 \mathrm{~Hz}, 1 \mathrm{H}), 7.46-7.58(\mathrm{~m}, 3 \mathrm{H})$.

${ }^{13} \mathbf{C}$ NMR $\left(\mathrm{CDCl}_{3}, 75 \mathrm{MHz}\right): \delta$ (ppm) 13.50, 13.91, 14.26, 14.79, 20.07, 22.81, 26.23, 29.38, 29.49, 29.56, 31.96, 40.32, 68.00, 112.1, 114.1, 114.6, 117.4, 120.2, 124.8, 126.8, 129.3, 130.8, 132.8, 137.1, 137.6, 138.6, $140.2,142.2,151.1,152.8,154.6,159.4$.

${ }^{19} \mathbf{F}$ NMR $\left(\mathrm{CDCl}_{3}, 376 \mathrm{MHz}\right): \delta(\mathrm{ppm})-143.01\left(\mathrm{q}, J_{\mathrm{B}-\mathrm{F}}=32.8 \mathrm{~Hz}\right)$.

HR-MS (ESI): $m / z$ calcd for $\mathrm{C}_{38} \mathrm{H}_{48} \mathrm{BF}_{2} \mathrm{~N}_{3} \mathrm{O}^{+} 611.3859$, found 611.3861 .

IR (ATR): $\tilde{v}\left(\mathrm{~cm}^{-1}\right)$ 808, 855, 949, 975, 1031, 1061, 1114, 1152, 1292, 1359, 1413, 1460, 1492, 1531, 1590 , $2854,2920$. 


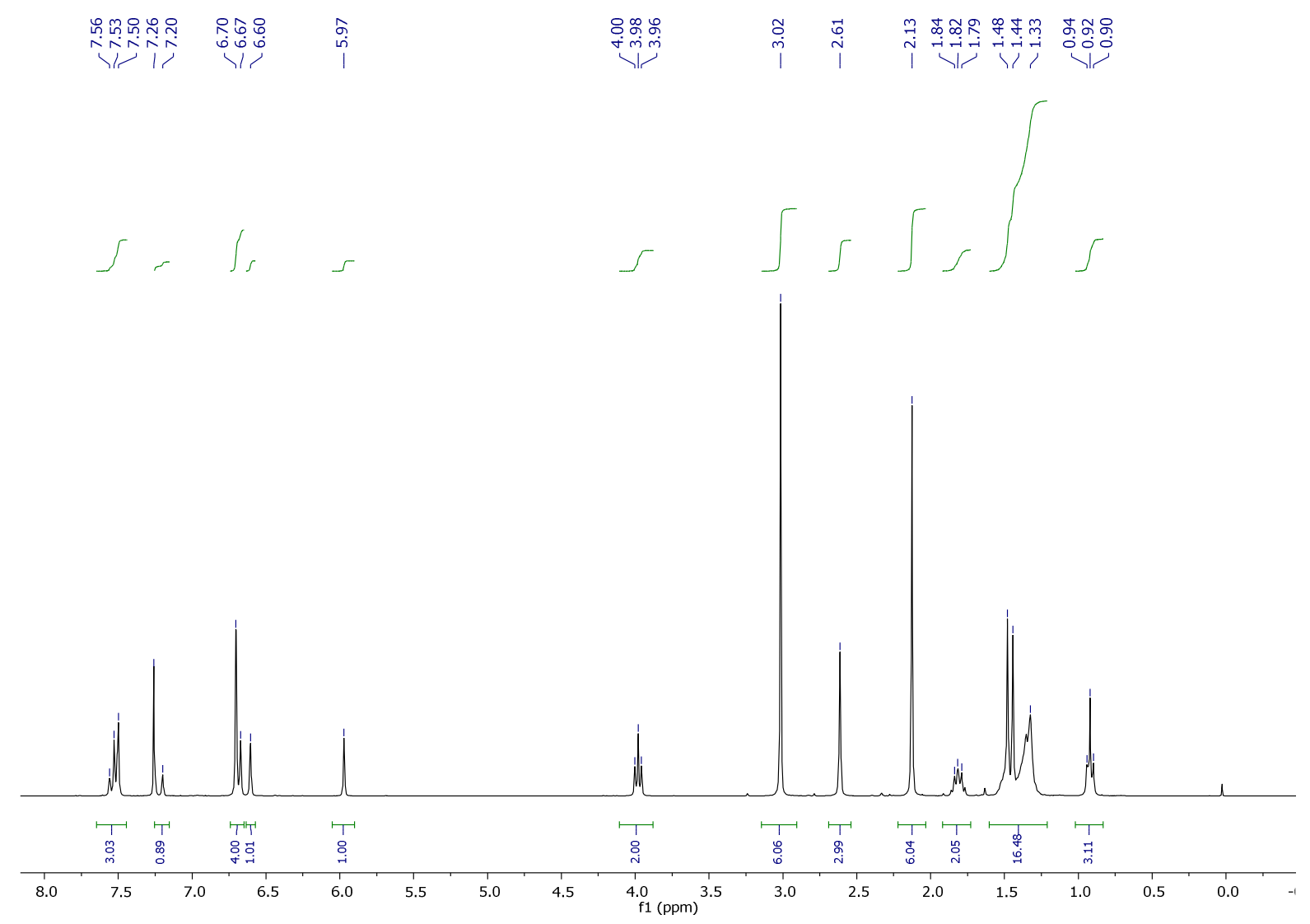

Figure S14. ${ }^{1} \mathrm{H}$ NMR $\left(\mathrm{CDCl}_{3}, 400 \mathrm{MHz}\right)$ spectrum of Red.

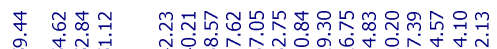

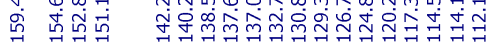

। 1।

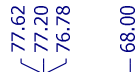

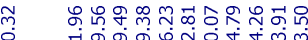

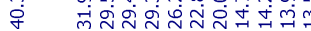

${ }^{19} \mathrm{~F} \mathrm{NMR}\left(\mathrm{CDCl}_{3}, 376 \mathrm{MHz}\right)$
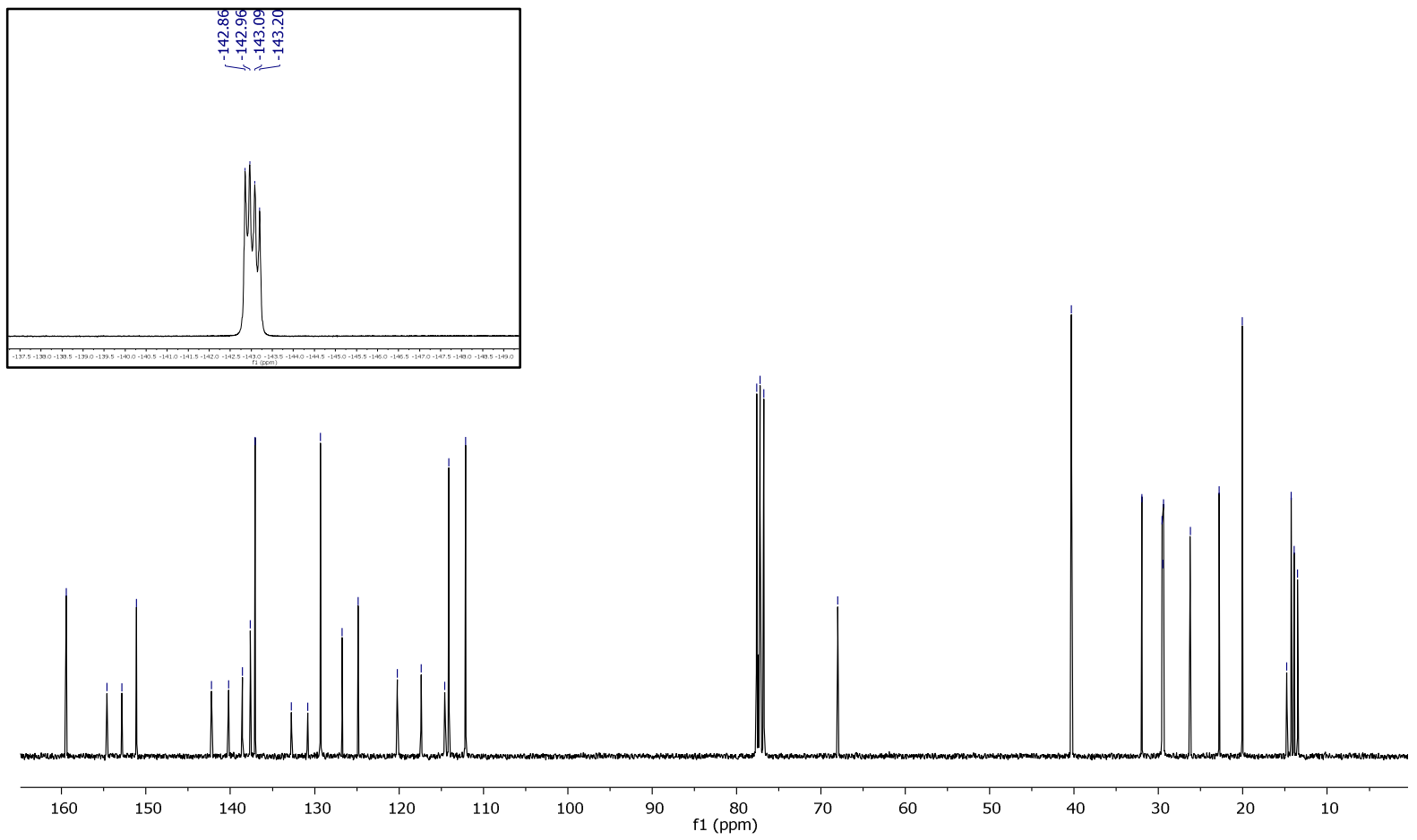

Figure S15. ${ }^{13} \mathrm{C} \mathrm{NMR}\left(\mathrm{CDCl}_{3}, 75 \mathrm{MHz}\right)$ spectrum of Red. 


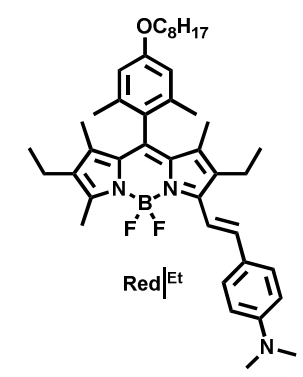

${ }^{1} \mathbf{H ~ N M R}\left(\mathrm{CDCl}_{3}, 300 \mathrm{MHz}\right): \delta(\mathrm{ppm}) 0.91(\mathrm{t}, J=7.4 \mathrm{~Hz}, 3 \mathrm{H}), 1.01(\mathrm{t}, J=7.6 \mathrm{~Hz}, 3 \mathrm{H}), 1.17(\mathrm{t}, J=7.5 \mathrm{~Hz}$, $3 \mathrm{H}), 1.25-1.55(\mathrm{~m}, 16 \mathrm{H}), 1.81(\mathrm{~m}, 2 \mathrm{H}), 2.10(\mathrm{~s}, 6 \mathrm{H}), 2.33(\mathrm{~m}, 2 \mathrm{H}), 2.58(\mathrm{~s}, 3 \mathrm{H}), 2.62(\mathrm{~m}, 2 \mathrm{H}), 3.01(\mathrm{~s}$, $6 \mathrm{H}), 3.98(\mathrm{t}, J=6.7 \mathrm{~Hz}, 2 \mathrm{H}), 6.69(\mathrm{~m}, 4 \mathrm{H}), 7.23(\mathrm{~d}, J=16.6 \mathrm{~Hz}, 1 \mathrm{H}), 7.51(\mathrm{~m}, 2 \mathrm{H}), 7.58(\mathrm{~d}, J=16.6 \mathrm{~Hz}$, $1 \mathrm{H})$.

${ }^{13} \mathbf{C}$ NMR $\left(\mathrm{CDCl}_{3}, 75 \mathrm{MHz}\right): \delta(\mathrm{ppm})$ 10.50, 10.80, 12.86, 14.29, 14.33, 14.91, 17.33, 18.62, 20.23, 22.85, 26.27, 29.43, 29.53, 29.60, 32.01, 40.52, 68.09, 112.3, 112.4, 114.1, 116.0, 126.1, 127.6, 128.8, 131.1, 131.7, $132.6,132.8,136.1,137.1,137.3,138.1,138.2,150.7,150.9,153.2,159.4$.

${ }^{19} \mathbf{F ~ N M R}\left(\mathrm{CDCl}_{3}, 376 \mathrm{MHz}\right): \delta(\mathrm{ppm})-143.15\left(\mathrm{q}, J_{\mathrm{B}-\mathrm{F}}=32.7 \mathrm{~Hz}\right)$.

HR-MS (ESI): $m / z$ calcd for $\mathrm{C}_{38} \mathrm{H}_{48} \mathrm{BF}_{2} \mathrm{~N}_{3} \mathrm{O}^{+} 667.4485$, found 667.4491 .

IR (ATR): $\tilde{v}\left(\mathrm{~cm}^{-1}\right)$ 959, 1001,1059, 1173, 1309, 1361, 1441, 1520, 1593, 1801, 2118, 2325, 2385, 2855, 2922.

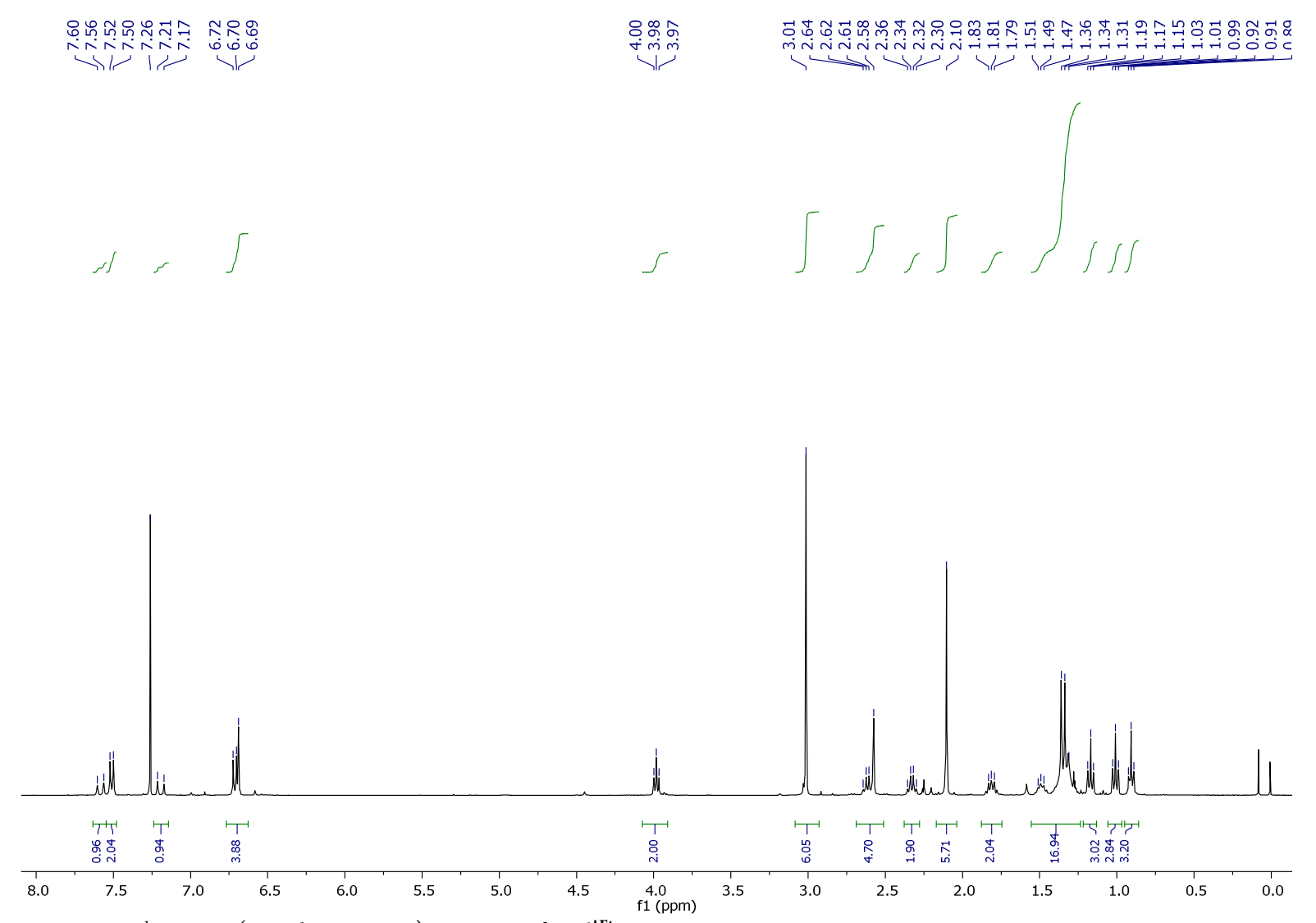

Figure S16. ${ }^{1} \mathrm{H} \mathrm{NMR}\left(\mathrm{CDCl}_{3}, 400 \mathrm{MHz}\right)$ spectrum of $\left.\mathbf{R e d}\right|^{\mathrm{Et}}$. 
${ }^{19} \mathrm{~F} \mathrm{NMR}\left(\mathrm{CDCl}_{3}, 376 \mathrm{MHz}\right)$
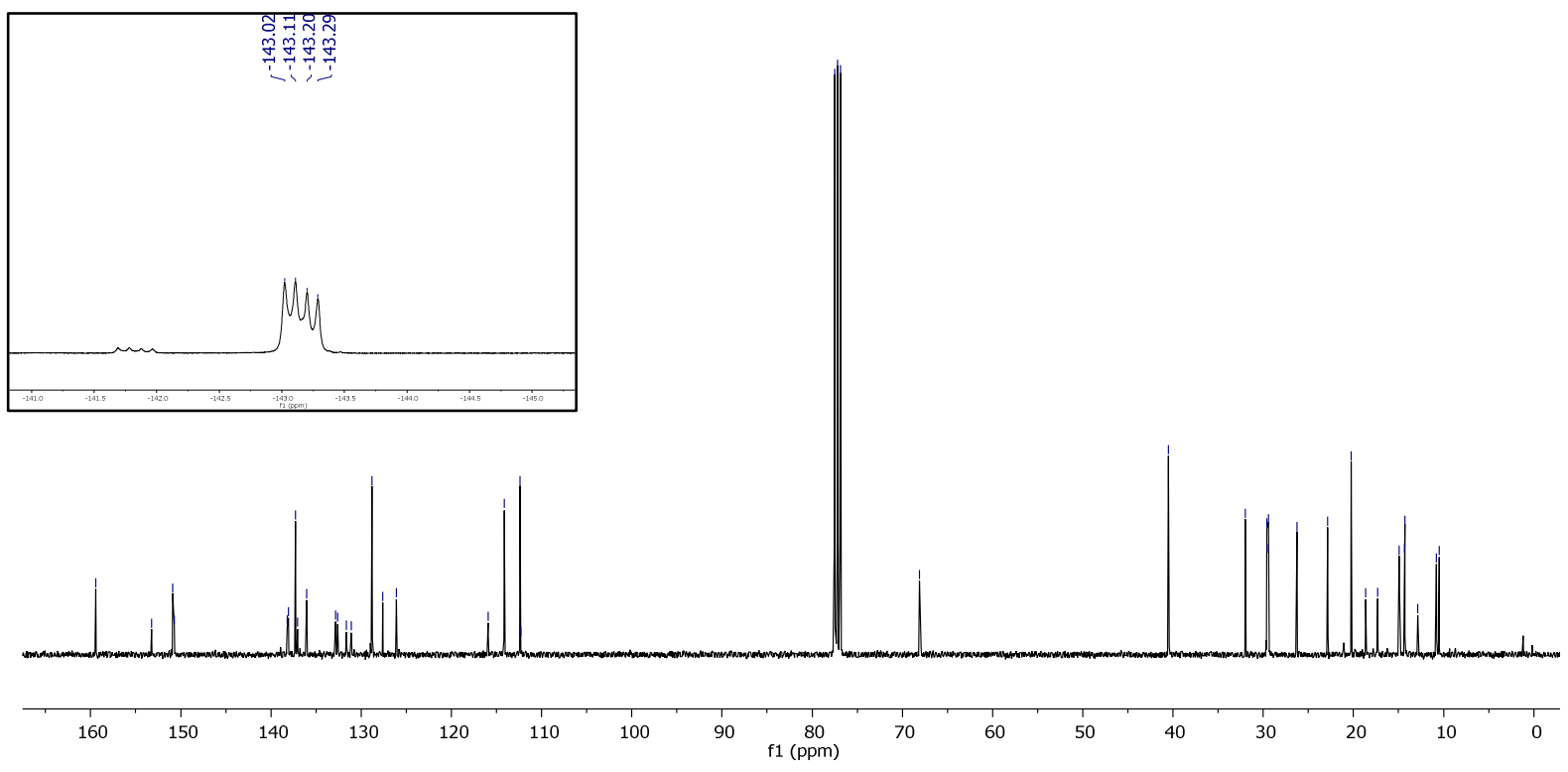

Figure S17. ${ }^{13} \mathrm{C} \mathrm{NMR}\left(\mathrm{CDCl}_{3}, 75 \mathrm{MHz}\right)$ spectrum of Red| $\left.\right|^{\mathrm{Et}}$.

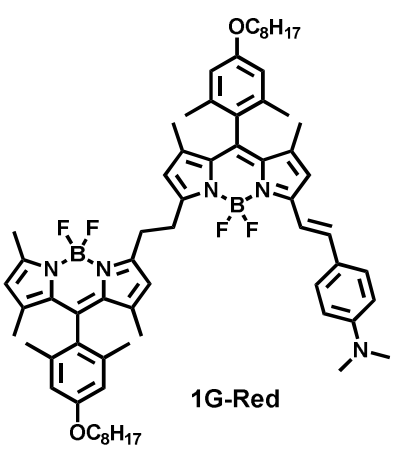

${ }^{1}$ H NMR $\left(\mathrm{CDCl}_{3}, 300 \mathrm{MHz}\right): \delta(\mathrm{ppm}) 0.91(\mathrm{t}, J=6.9 \mathrm{~Hz}, 6 \mathrm{H}), 1.20-1.56(\mathrm{~m}, 32 \mathrm{H}), 1.81(\mathrm{~m}, 4 \mathrm{H}), 2.12(\mathrm{~s}$, $12 \mathrm{H}), 2.57$ (s, $3 \mathrm{H}), 3.01$ (s, $6 \mathrm{H}), 3.45$ (s, $4 \mathrm{H}), 3.97$ (m, $4 \mathrm{H}), 5.97$ (s, $1 \mathrm{H}), 6.15(\mathrm{~s}, 1 \mathrm{H}), 6.21(\mathrm{~s}, 1 \mathrm{H}), 6.59$ $(\mathrm{s}, 1 \mathrm{H}), 6.65-6.72(\mathrm{~m}, 6 \mathrm{H}), 7.21(\mathrm{~d}, J=16.2 \mathrm{~Hz}, 1 \mathrm{H}), 7.46-7.54(\mathrm{~m}, 3 \mathrm{H})$.

${ }^{13} \mathrm{C}$ NMR $\left(\mathrm{CDCl}_{3}, 75 \mathrm{MHz}\right): \delta$ (ppm) 13.67, 13.69, 13.83, 13.98, 14.29, 14.85, 20.08, 20.16, 22.85, 26.27, 27.12, 27.20, 29.42, 29.53, 29.60, 32.00, 40.40, 68.08, 112.2, 114.2, 114.3, 114.7, 117.5, 118.9, 119.8, 121.0, 125.0, 126.6, 126.9, 129.4, 130.8, 131.1, 131.2, 132.9, 136.8, 137.1, 137.7, 138.9, 140.3, 141.8, 142.4, 142.5, $151.2,154.8,155.3,155.8,158.5,159.5,159.6$.

${ }^{19} \mathbf{F}$ NMR $\left(\mathrm{CDCl}_{3}, 376 \mathrm{MHz}\right): \delta(\mathrm{ppm})-141.84\left(\mathrm{q}, J_{\mathrm{B}-\mathrm{F}}=31.7 \mathrm{~Hz}, 2 \mathrm{~F}\right),-145.57\left(\mathrm{q}, J_{\mathrm{B}-\mathrm{F}}=32.3 \mathrm{~Hz}, 2 \mathrm{~F}\right)$.

HR-MS (ESI): $m / z$ calcd for $\mathrm{C}_{67} \mathrm{H}_{85} \mathrm{~B}_{2} \mathrm{~F}_{4} \mathrm{~N}_{5} \mathrm{O}_{2}{ }^{+} 1089.6826$, found 1089.6859 .

IR (ATR): $\tilde{v}\left(\mathrm{~cm}^{-1}\right)$ 977, 1030, 1073, 1108, 1152, 1178, 1208, 1301, 1362, 1448, 1494, 1537, 1592, 2027, 2161, 2856, 2923. 


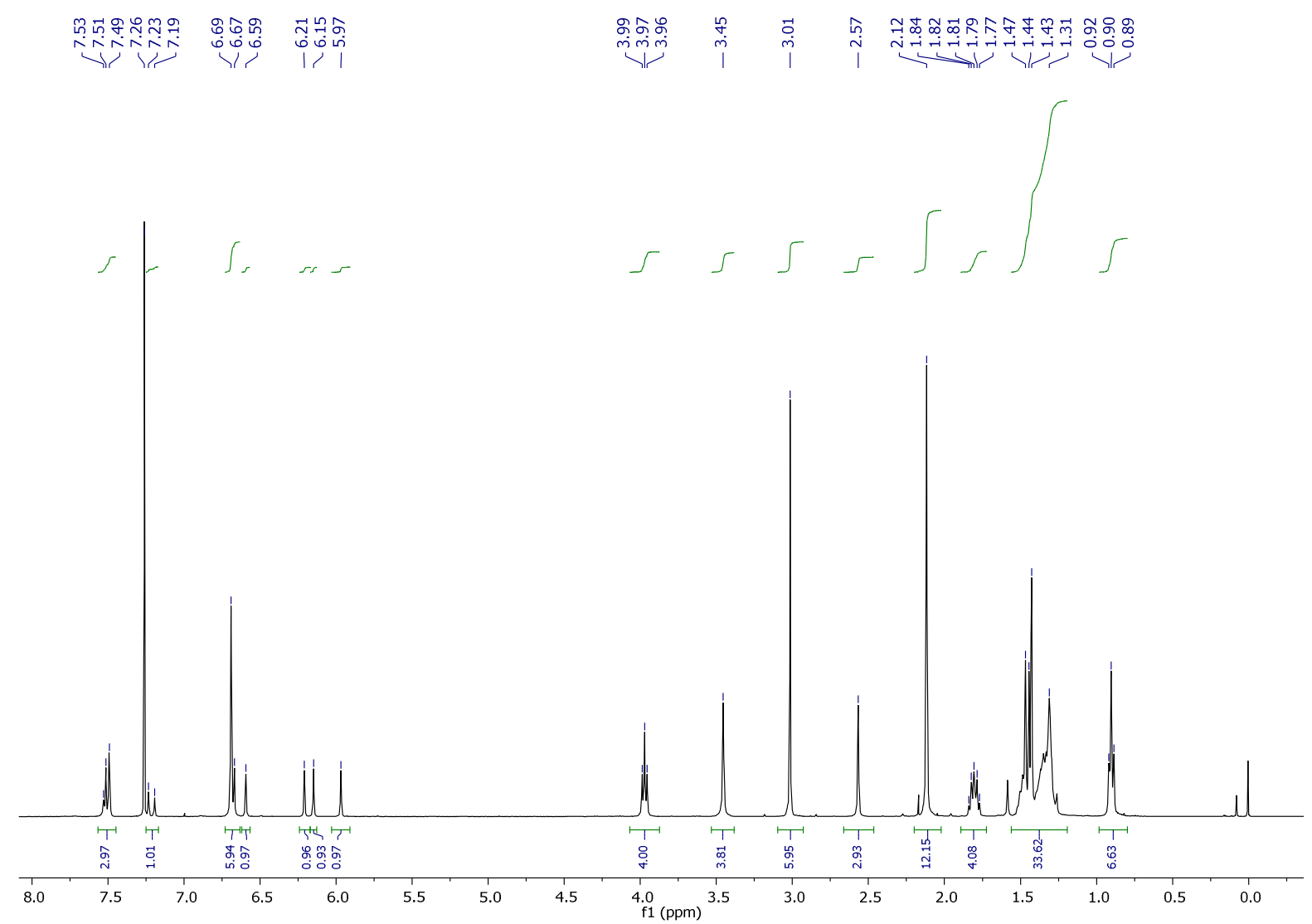

Figure S18. ${ }^{1} \mathrm{H}$ NMR $\left(\mathrm{CDCl}_{3}, 400 \mathrm{MHz}\right)$ spectrum of 1G-Red.

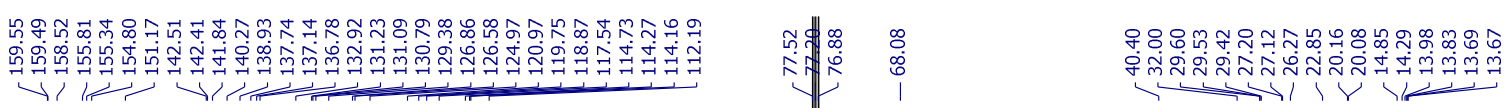

${ }^{19} \mathrm{~F} \mathrm{NMR}\left(\mathrm{CDCl}_{3}, 376 \mathrm{MHz}\right)$
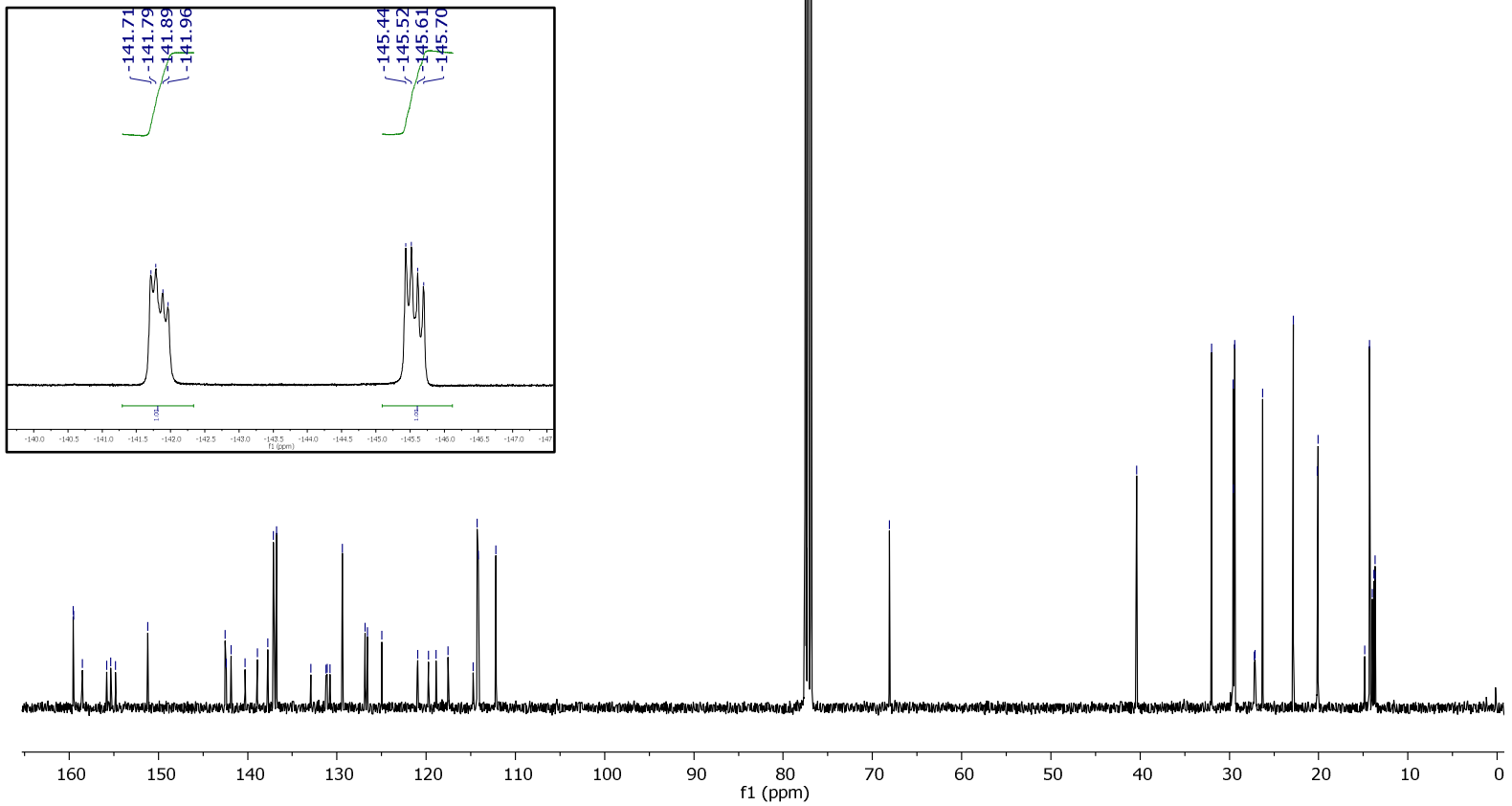

Figure S19. ${ }^{13} \mathrm{C} \mathrm{NMR}\left(\mathrm{CDCl}_{3}, 100 \mathrm{MHz}\right)$ spectrum of $\mathbf{1 G - R e d}$. 


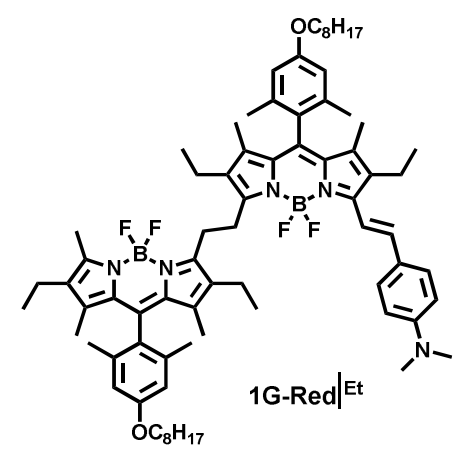

${ }^{1} \mathbf{H}$ NMR $\left(\mathrm{CDCl}_{3}, 300 \mathrm{MHz}\right): \delta(\mathrm{ppm}) 0.91(\mathrm{t}, J=6.9 \mathrm{~Hz}, 6 \mathrm{H}), 1.00(\mathrm{t}, J=7.5 \mathrm{~Hz}, 3 \mathrm{H}), 1.05(\mathrm{t}, J=7.4 \mathrm{~Hz}$, $3 \mathrm{H}), 1.10(\mathrm{t}, J=7.5 \mathrm{~Hz}, 3 \mathrm{H}), 1.16(\mathrm{t}, J=7.5 \mathrm{~Hz}, 3 \mathrm{H}), 1.20-1.56(\mathrm{~m}, 28 \mathrm{H}), 1.49(\mathrm{~m}, 4 \mathrm{H}), 1.81(\mathrm{~m}, 4 \mathrm{H})$, $2.11(\mathrm{~s}, 12 \mathrm{H}), 2.32(\mathrm{q}, J=7.4 \mathrm{~Hz}, 2 \mathrm{H}), 2.50(\mathrm{~m}, 4 \mathrm{H}), 2.55(\mathrm{~s}, 3 \mathrm{H}), 2.61(\mathrm{q}, J=7.5 \mathrm{~Hz}, 2 \mathrm{H}), 3.02(\mathrm{~s}, 6 \mathrm{H})$, $3.40(\mathrm{~s}, 4 \mathrm{H}), 3.98(\mathrm{~m}, 4 \mathrm{H}), 5.97(\mathrm{~s}, 1 \mathrm{H}), 6.65-6.72(\mathrm{~m}, 6 \mathrm{H}), 7.22(\mathrm{~d}, J=16.6 \mathrm{~Hz}, 1 \mathrm{H}), 7.50(\mathrm{~m}, 2 \mathrm{H}), 7.61$ $(\mathrm{d}, J=16.6 \mathrm{~Hz}, 1 \mathrm{H})$.

${ }^{13} \mathbf{C}$ NMR $\left(\mathrm{CDCl}_{3}, 75 \mathrm{MHz}\right): \delta(\mathrm{ppm}) 10.51,10.81,10.92,10.98,12.74,14.31,14.35,14.93,15.28,15.38$, $16.65,17.29,18.61,20.21,20.28,22.87,26.28,27.35,27.51,29.45,29.55,29.62,29.89,32.02,40.55,68.10$, $112.4,114.1,114.2,116.1,126.3,127.5,127.7,128.7,130.5,130.7,131.4,131.8,132.6,133.0,133.8,134.0$, $135.9,137.1,137.3,137.7,138.2,138.3,138.4,140.0,150.5,150.8,153.5,156.2,156.5,159.4$.

${ }^{19} \mathbf{F ~ N M R}\left(\mathrm{CDCl}_{3}, 376 \mathrm{MHz}\right): \delta$ (ppm) -140.29 (m, $\left.2 \mathrm{~F}\right),-143.56$ (m, $\left.2 \mathrm{~F}\right)$.

HR-MS (ESI): $m / z$ calcd for $\mathrm{C}_{75} \mathrm{H}_{101} \mathrm{~B}_{2} \mathrm{~F}_{4} \mathrm{~N}_{5} \mathrm{O}_{2}{ }^{+} 1201.8076$, found 1201.8105 .

IR (ATR): $\tilde{v}\left(\mathrm{~cm}^{-1}\right)$ 701, 730, 768, 809, 857, 936, 967, 1019, 1046, 1114, 1136, 1177, 1204, 1268, 1318, 1361, 1384, 1408, 1443, 1529, 1598, 2858, 2924.

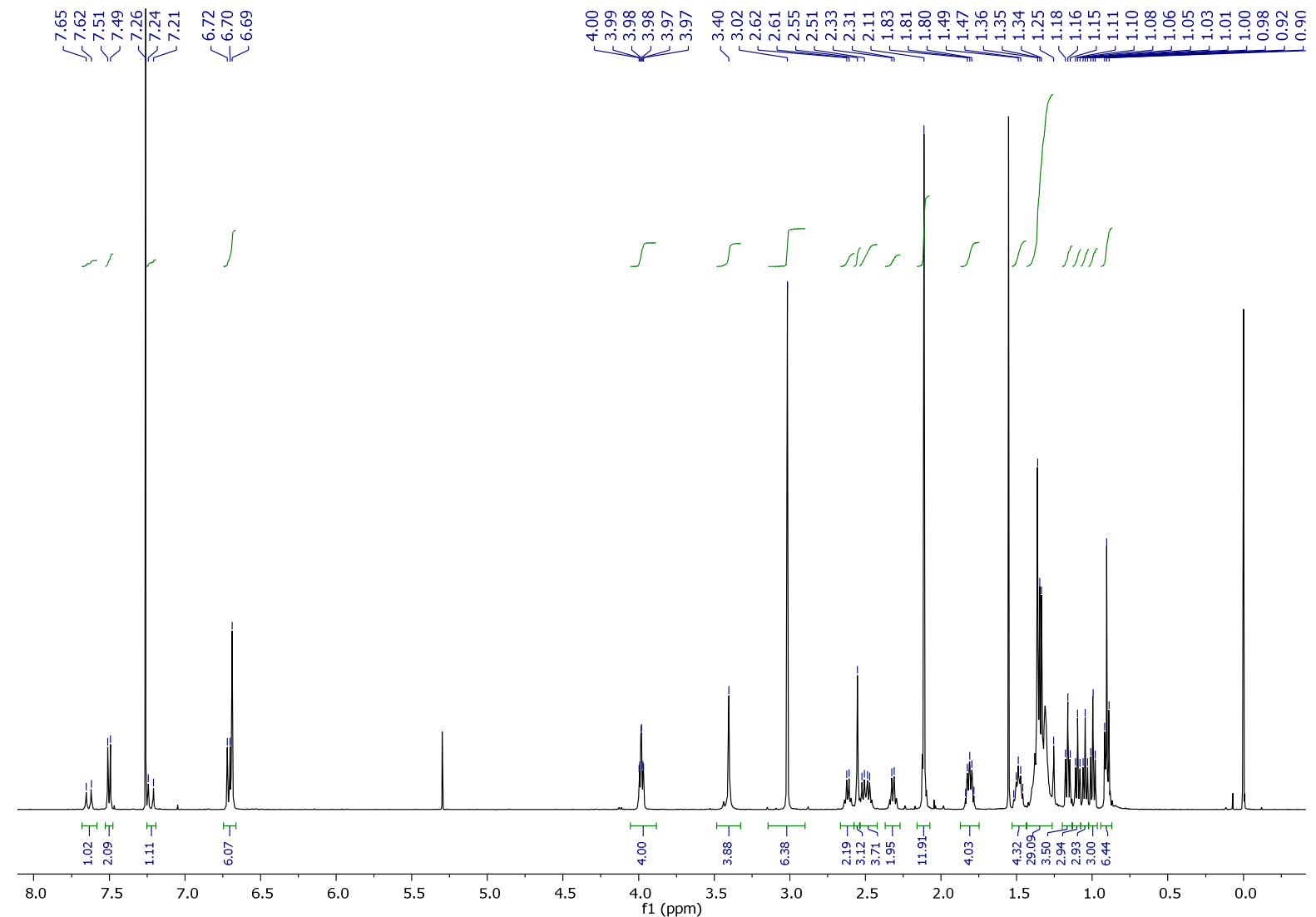

Figure S20. ${ }^{1} \mathrm{H} \mathrm{NMR}\left(\mathrm{CDCl}_{3}, 400 \mathrm{MHz}\right)$ spectrum of $\left.\mathbf{1 G - R e d}\right|^{\text {Et }}$. 


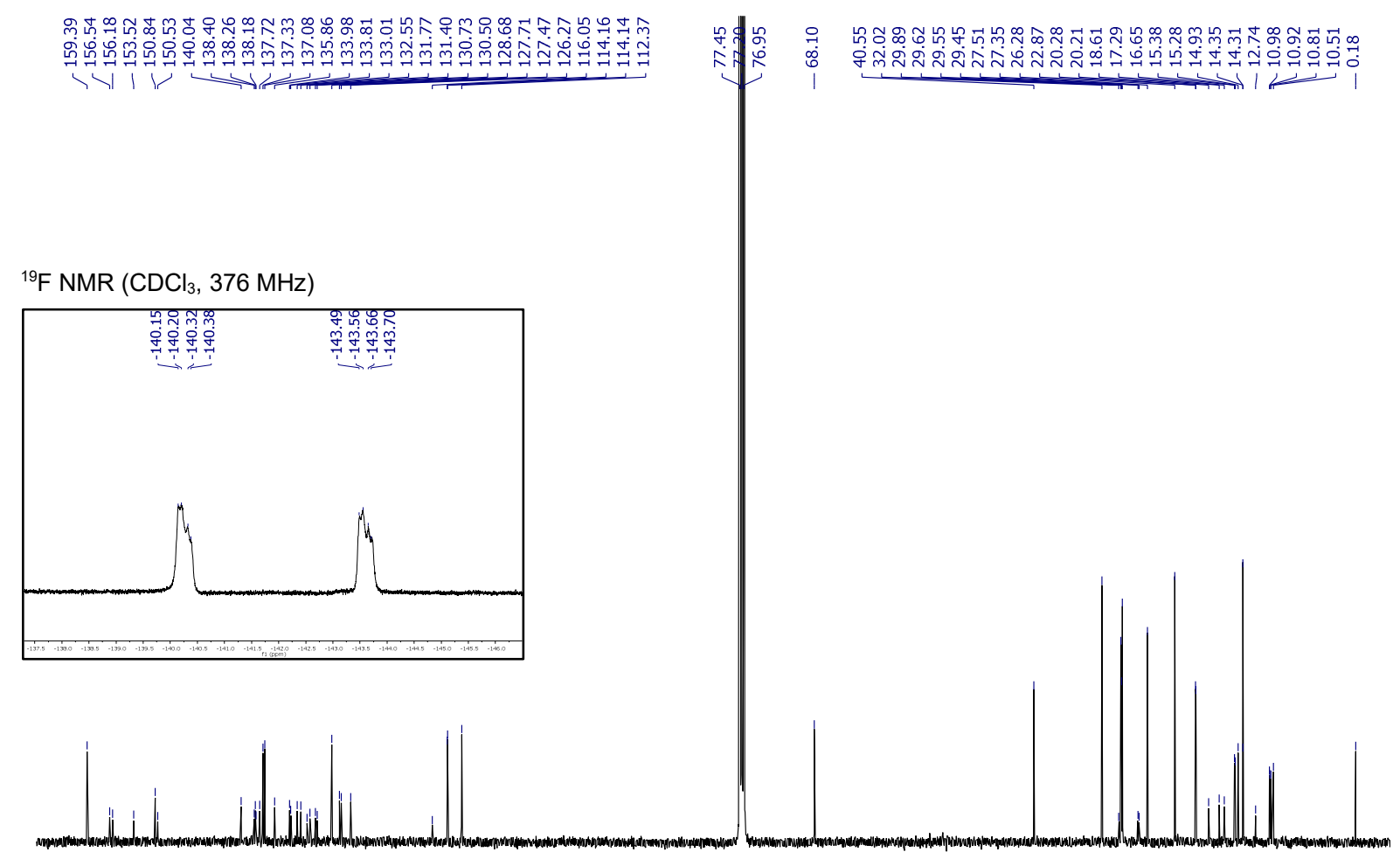

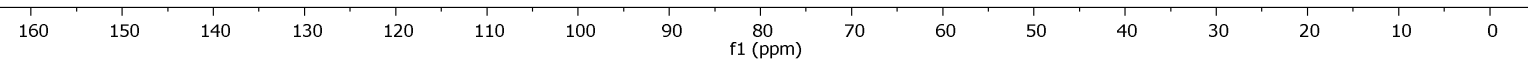

Figure S21. ${ }^{13} \mathrm{C} \mathrm{NMR}\left(\mathrm{CDCl}_{3}, 125 \mathrm{MHz}\right)$ spectrum of $\left.\mathbf{1 G - R e d}\right|^{\mathrm{Et}}$.

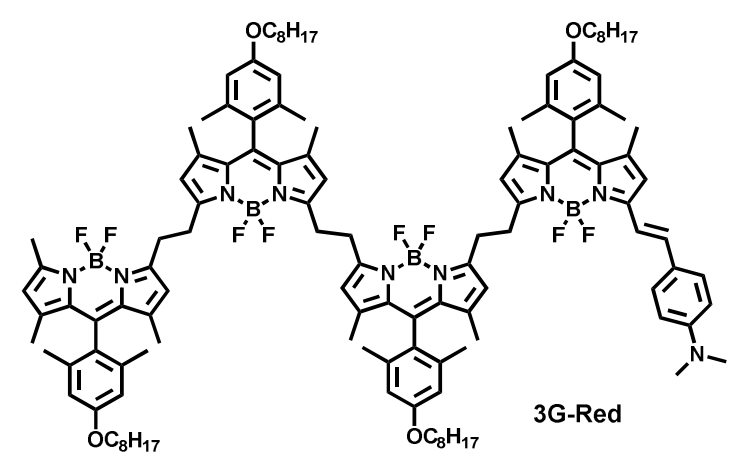

${ }^{1} \mathbf{H}$ NMR $\left(\mathrm{CDCl}_{3}, 300 \mathrm{MHz}\right): \delta(\mathrm{ppm}) 0.90(\mathrm{~m}, 12 \mathrm{H}), 1.20-1.53(\mathrm{~m}, 64 \mathrm{H}), 1.80(\mathrm{~m}, 8 \mathrm{H}), 2.09(\mathrm{~s}, 6 \mathrm{H})$, $2.10(\mathrm{~s}, 6 \mathrm{H}), 2.11(\mathrm{~s}, 6 \mathrm{H}), 2.11(\mathrm{~s}, 6 \mathrm{H}), 2.55(\mathrm{~s}, 3 \mathrm{H}), 3.01(\mathrm{~s}, 6 \mathrm{H}), 3.35-3.50(\mathrm{~m}, 12 \mathrm{H}), 3.96(\mathrm{~m}, 8 \mathrm{H})$, $5.96(\mathrm{~s}, 1 \mathrm{H}), 6.14(\mathrm{~s}, 1 \mathrm{H}), 6.16(\mathrm{~s}, 2 \mathrm{H}), 6.18(\mathrm{~s}, 1 \mathrm{H}), 6.20(\mathrm{~s}, 1 \mathrm{H}), 6.58(\mathrm{~s}, 1 \mathrm{H}), 6.68(\mathrm{~m}, 10 \mathrm{H}), 7.20(\mathrm{~d}, J$ $=16.2 \mathrm{~Hz}, 1 \mathrm{H}), 7.50(\mathrm{~m}, 3 \mathrm{H})$.

${ }^{13} \mathrm{C}$ NMR $\left(\mathrm{CDCl}_{3}, 75 \mathrm{MHz}\right): \delta$ (ppm) 13.70, 13.71, 13.81, 13.84, 13.85, 13.89, 14.00, 14.26, 14.32, 14.88, $20.08,20.11,20.14,20.19,22.87,26.28,27.03,27.12,27.21,29.44,29.53,29.54,29.61,32.02,40.43,68.07$, 76.99, 77.41, 112.2, 114.1, 114.3, 117.5, 118.9, 119.6, 121.0, 125.0, 126.52, 126.56, 126.6, 126.9, 129.4, 131.0, 131.2 , 136.75, 136.76, 136.8, 137.2, 137.7, 138.9, 142.0, 142.2, 142.3, 142.47, 142.52, 142.7, 151.2, 155.5 , $158.1,159.46,159.53,159.54$.

${ }^{19} \mathbf{F}$ NMR $\left(\mathrm{CDCl}_{3}, 376 \mathrm{MHz}\right): \delta$ (ppm) -141.90 (m, $\left.2 \mathrm{~F}\right),-144.32$ (m, $\left.4 \mathrm{~F}\right),-145.61$ (m, $\left.2 \mathrm{~F}\right)$.

HR-MS (ESI): $m / z$ calcd for $\mathrm{C}_{125} \mathrm{H}_{159} \mathrm{~B}_{4} \mathrm{~F}_{8} \mathrm{~N}_{9} \mathrm{O}_{4} \mathrm{H}^{+}$2047.2832, found 2047.2864.

IR (ATR): $\tilde{v}\left(\mathrm{~cm}^{-1}\right)$ 699, 810, 853, 976, 1029, 1074, 1101, 1149, 1180, 1209, 1305, 1362, 1445, 1497, 1539, 1596, 2117, 2326, 2386, 2856, 2923. 


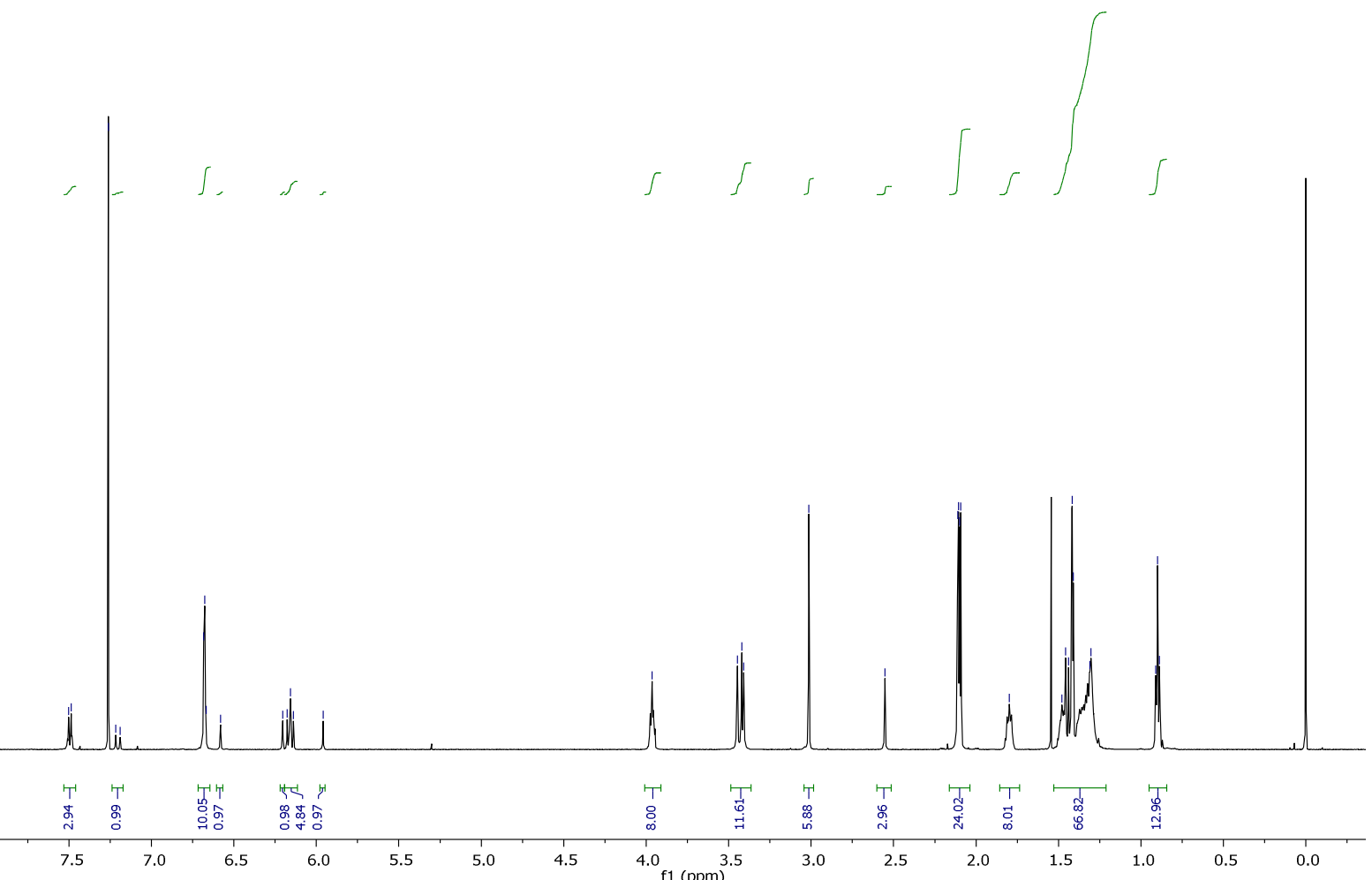

Figure S22. ${ }^{1} \mathrm{H}$ NMR $\left(\mathrm{CDCl}_{3}, 600 \mathrm{MHz}\right)$ spectrum of 3G-Red.

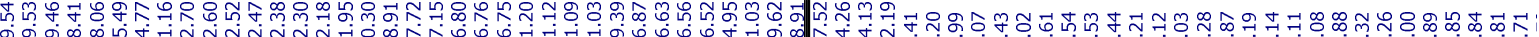

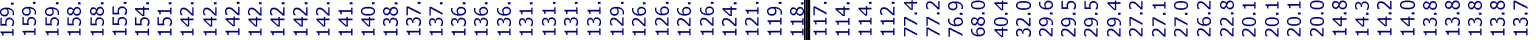

${ }^{19} \mathrm{~F} \mathrm{NMR}\left(\mathrm{CDCl}_{3}, 376 \mathrm{MHz}\right)$
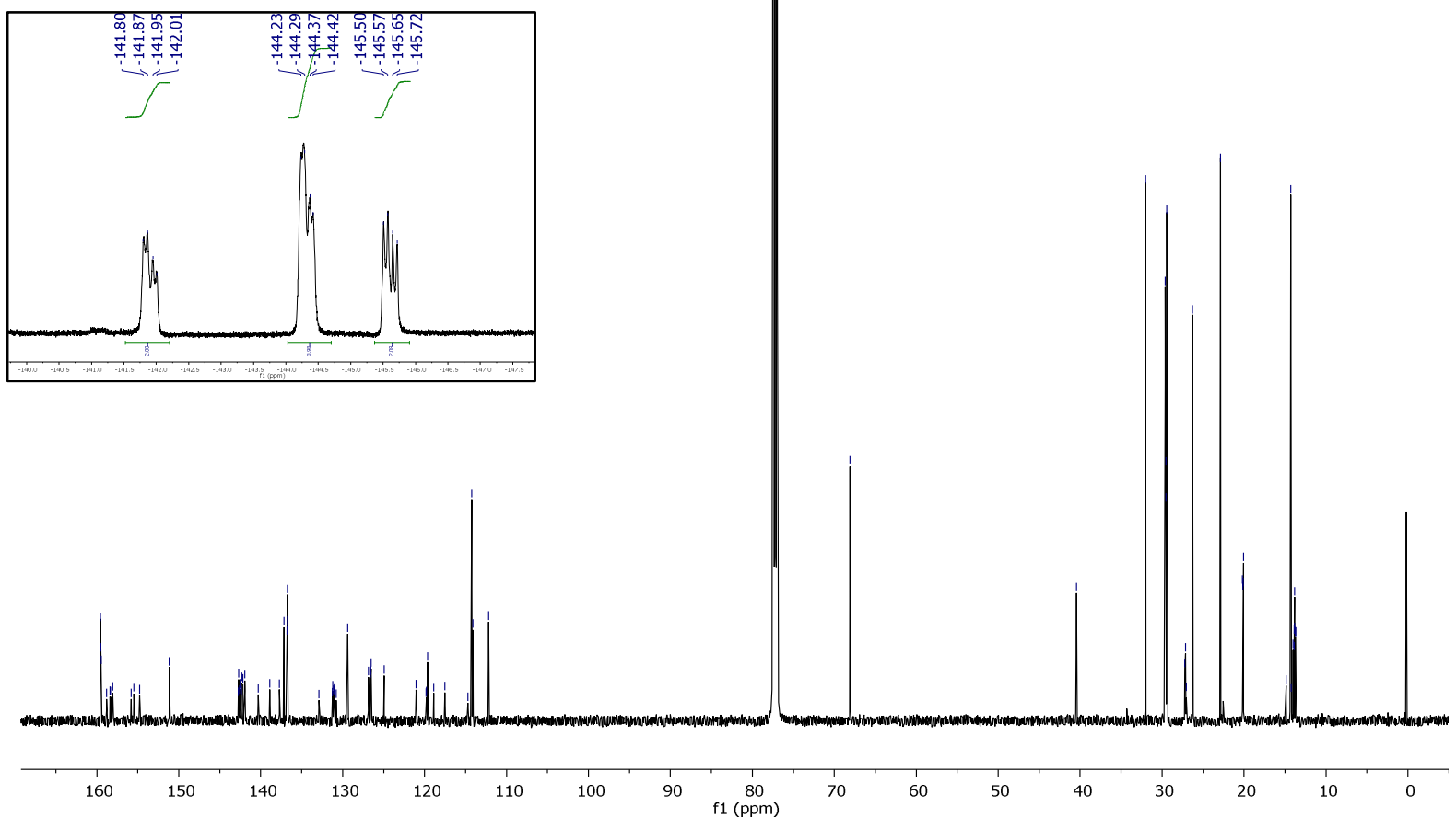

Figure S23. ${ }^{13} \mathrm{C}$ NMR $\left(\mathrm{CDCl}_{3}, 150 \mathrm{MHz}\right)$ spectrum of 3G-Red. 


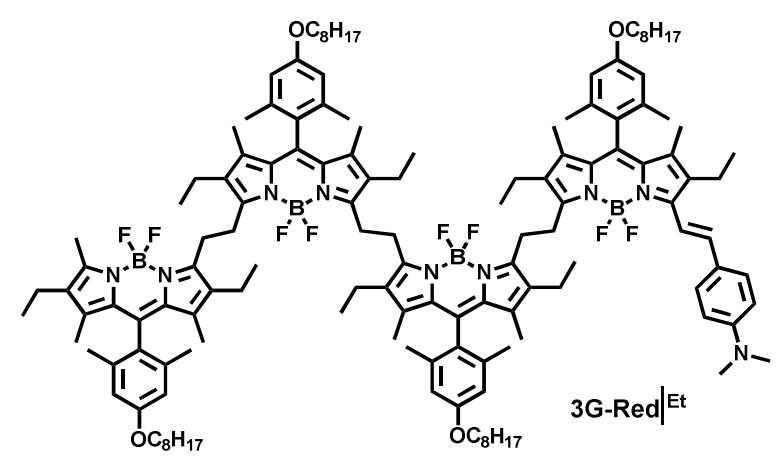

${ }^{1}$ H NMR $\left(\mathrm{CDCl}_{3}, 300 \mathrm{MHz}\right): \delta(\mathrm{ppm}) 0.80-1.10(\mathrm{~m}, 33 \mathrm{H}), 1.16(\mathrm{t}, J=7.4 \mathrm{~Hz}, 3 \mathrm{H}), 1.28-1.53(\mathrm{~m}, 64 \mathrm{H})$, $1.81(\mathrm{~m}, 8 \mathrm{H}), 2.11(\mathrm{~m}, 24 \mathrm{H}), 2.20-2.50(\mathrm{~m}, 14 \mathrm{H}), 2.56(\mathrm{~s}, 3 \mathrm{H}), 2.62\left(\mathrm{~s}_{\mathrm{br}}, 2 \mathrm{H}\right), 3.03\left(\mathrm{~s}_{\mathrm{br}}, 3 \mathrm{H}\right), 3.35-3.55$ $(\mathrm{m}, 12 \mathrm{H}), 3.98(\mathrm{~m}, 8 \mathrm{H}), 6.69(\mathrm{~m}, 10 \mathrm{H}), 7.21\left(\mathrm{~s}_{\mathrm{br}}, 1 \mathrm{H}\right), 7.51(\mathrm{~m}, 2 \mathrm{H}) 7.63\left(\mathrm{~s}_{\mathrm{br}}, 1 \mathrm{H}\right)$.

${ }^{13}$ C NMR $\left(\mathrm{CDCl}_{3}, 75 \mathrm{MHz}\right): \delta$ (ppm) 10.81, 10.92, 11.01, 12.76, 14.31, 14.92, 15.11, 15.13, 15.16, 15.29, $16.59,20.15$, 20.17, 20.27, 22.87, 26.27, 27.18, 29.44, 29.53, 29.61, 32.02, 68.10, 114.2, 127.4, 127.55, 127.59, $127.7,130.5,130.7,130.9,132.6,133.86,133.94,134.03,134.07,137.02,137.06,137.3,137.8,138.2,138.3$, 138.4, 140.1, 140.2, 140.3, 153.6, 156.4, 156.5, 156.7, 156.8, 159.4 .

${ }^{19}$ F NMR $\left(\mathrm{CDCl}_{3}, 376 \mathrm{MHz}\right): \delta(\mathrm{ppm})-140.26$ (m, $\left.2 \mathrm{~F}\right),-141.03$ (m, $\left.4 \mathrm{~F}\right),-143.64(\mathrm{~m}, 2 \mathrm{~F})$.

HR-MS (ESI): $m / z$ calcd for $\mathrm{C}_{141} \mathrm{H}_{191} \mathrm{~B}_{4} \mathrm{~F}_{8} \mathrm{~N}_{9} \mathrm{O}_{4} \mathrm{H}^{+} 2271.5336$, found 2271.5328 .

IR (ATR): $\tilde{v}\left(\mathrm{~cm}^{-1}\right) 700,731,766,809,856,933,964,1037,1120,1176,1203,1268,1315,1364,1385,1411$, 1443, 1528, 1600, 1802, 1995, 2118, 2325, 2385, 2668, 2858, 2924.

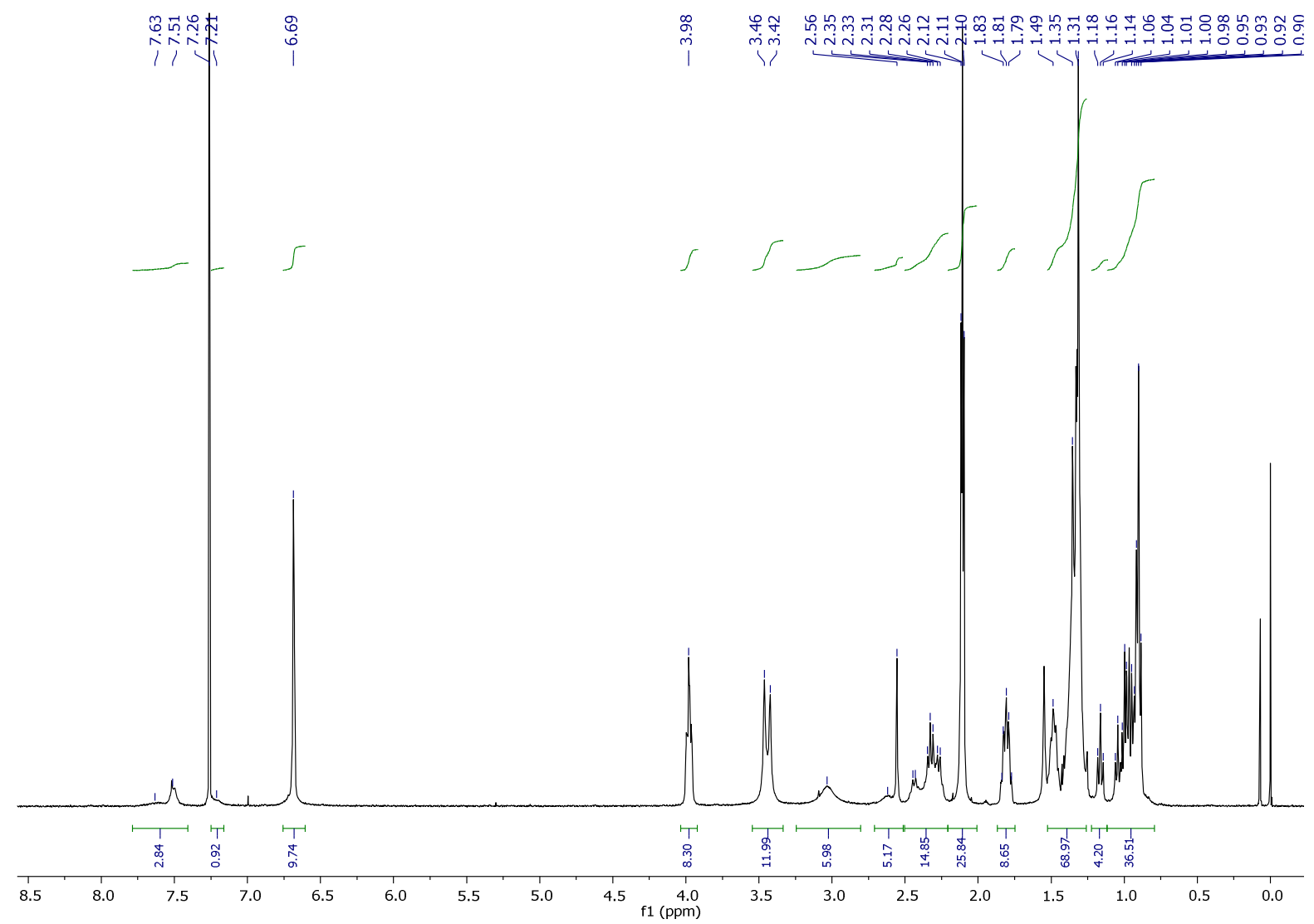

Figure S24. ${ }^{1} \mathrm{H}$ NMR $\left(\mathrm{CDCl}_{3}, 600 \mathrm{MHz}\right)$ spectrum of 3G-Red $\left.\right|^{\mathrm{Et}}$. 


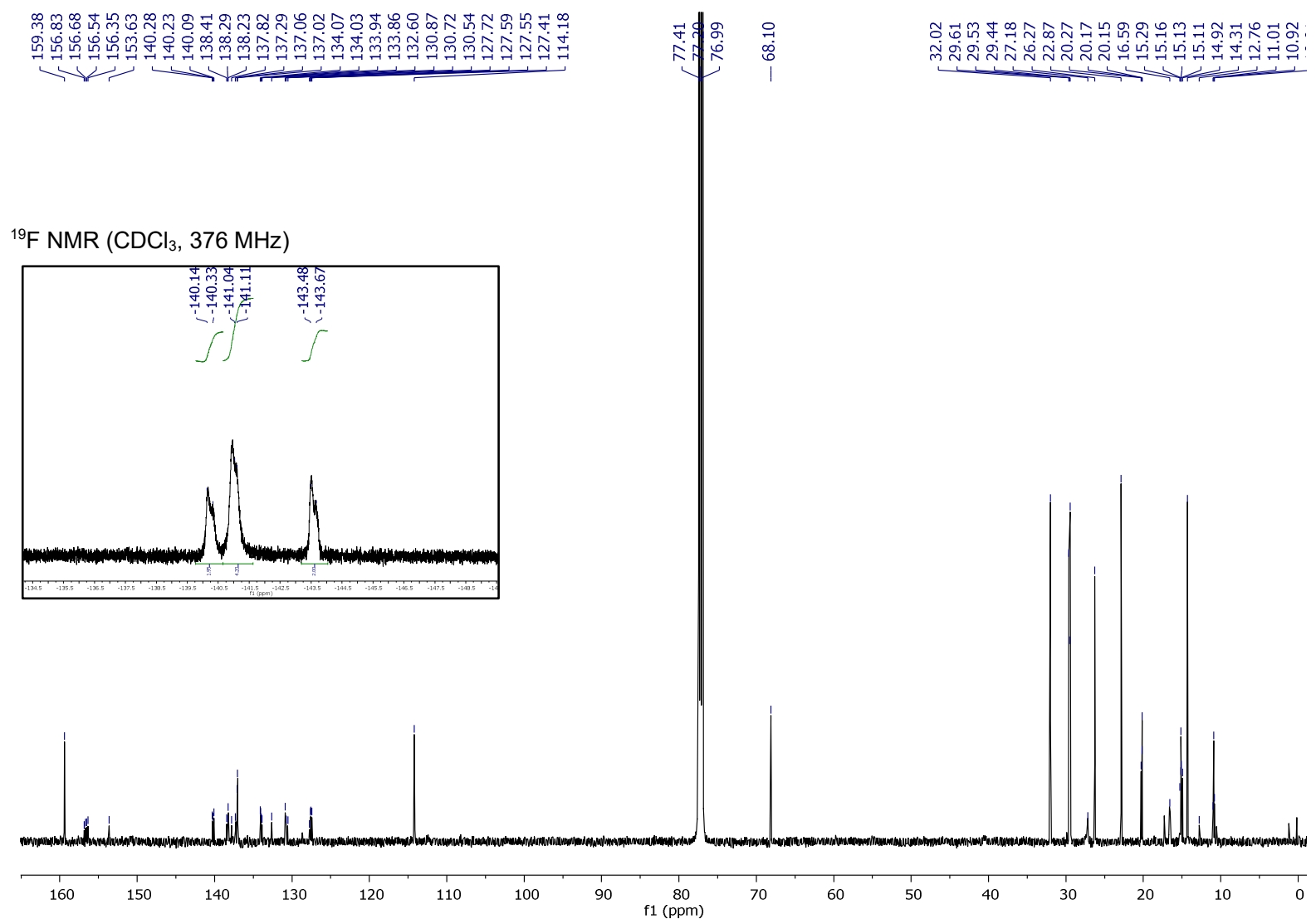

Figure S25. ${ }^{13} \mathrm{C} \mathrm{NMR}\left(\mathrm{CDCl}_{3}, 125 \mathrm{MHz}\right)$ spectrum of 3G-Red $\left.\right|^{\mathrm{Et}}$.

\section{Calculation of Transition Dipole Moments}

Transition dipole moments were obtained from equation $S 1^{9}$ with $h$ being Planck's constant, $c$ is the speed of light $\left[3 \times 10^{10} \mathrm{~cm} / \mathrm{s}\right], \varepsilon_{0}$ is the electric field constant, $n$ is the refractive index of the solvent, $N_{\mathrm{Av}}$ is the Avogadro constant and $\varepsilon(\tilde{v})$ is the attenuation coefficient as a function of the wavenumber $\tilde{v}$ (except for $c, \varepsilon(\tilde{v})$ and $\tilde{v}$, all constants in SI units).

(S1) $\mu_{\mathrm{eg}}^{2}=\frac{3 h c \varepsilon_{0} \cdot \ln (10) \cdot 9 n}{2000 \pi^{2} \cdot N_{A v} \cdot\left(n^{2}+2\right)^{2}} \cdot \int \frac{\varepsilon(\tilde{v}) d \tilde{v}}{\tilde{v}}$ 


\section{Transient Absorption Spectra}

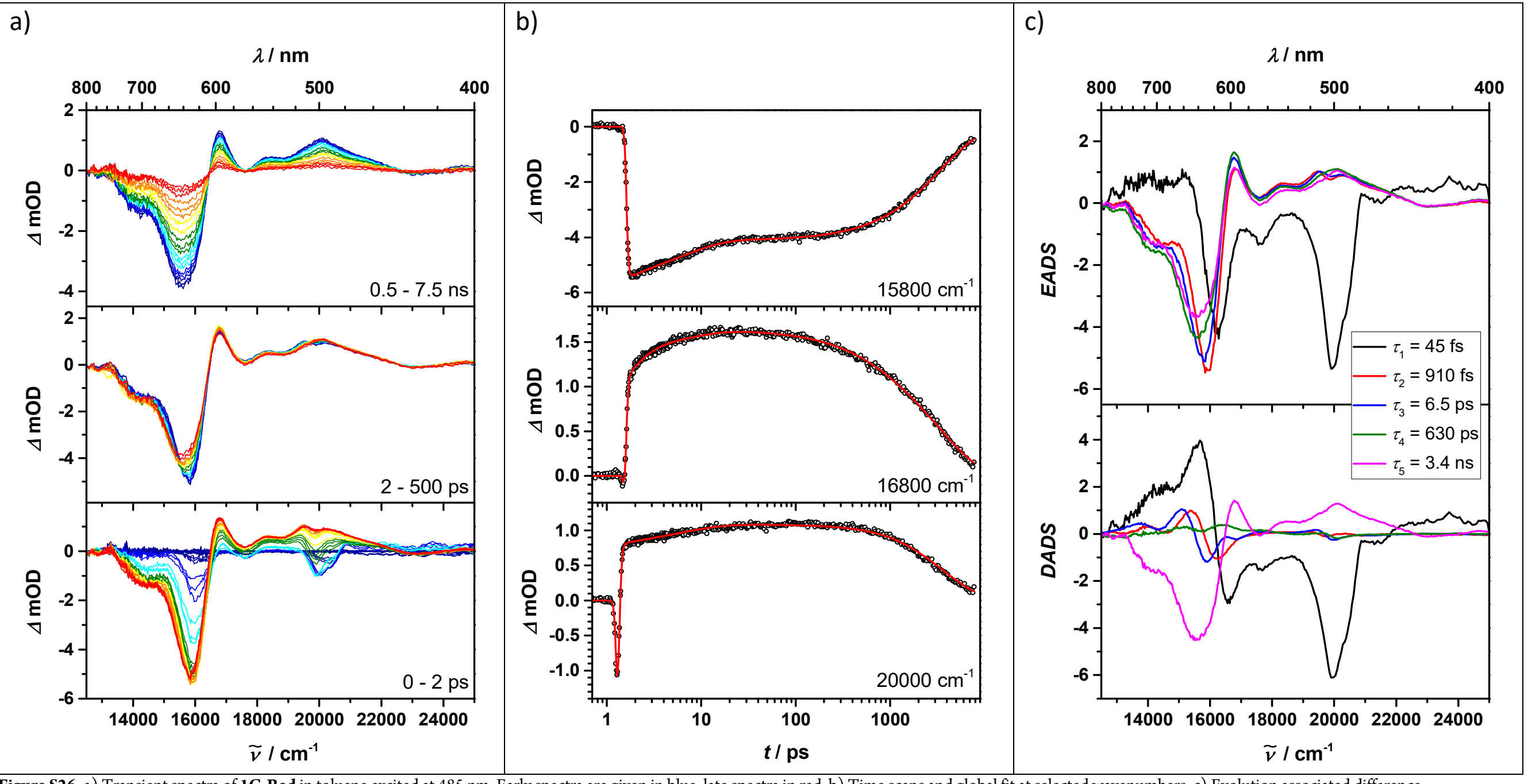

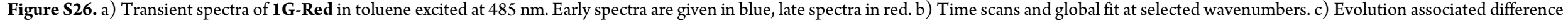
spectra (top) and decay associated difference spectra (bottom) of a global fit using GLOTARAN software. 

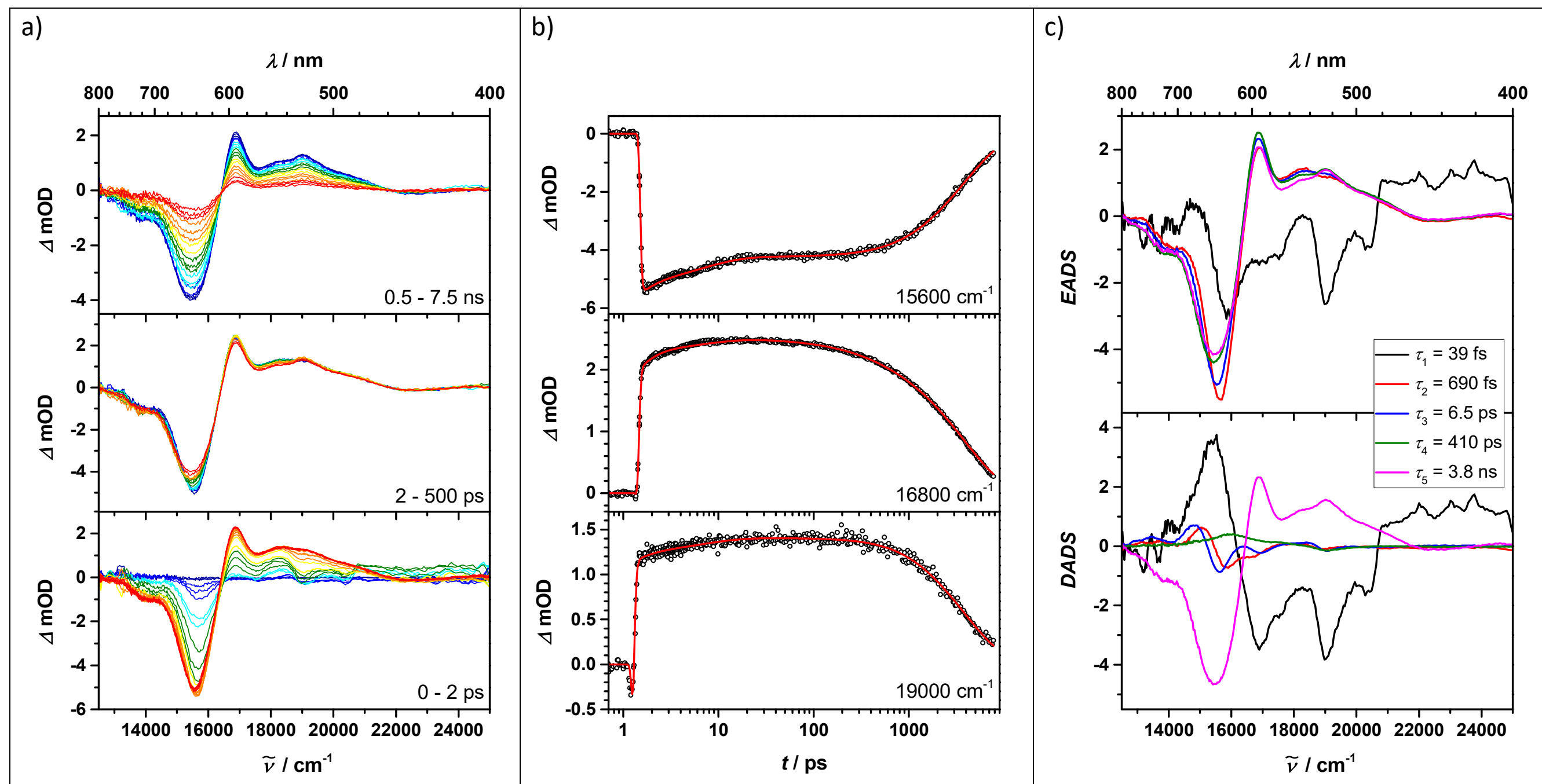

Figure S27. a) Transient spectra of 1G-Red| ${ }^{\mathrm{Et}}$ in toluene excited at $485 \mathrm{~nm}$. Early spectra are given in blue, late spectra in red. b) Time scans and global fit at selected wavenumbers. c) Evolution associated difference spectra (top) and decay associated difference spectra (bottom) of a global fit using GLOTARAN software. 


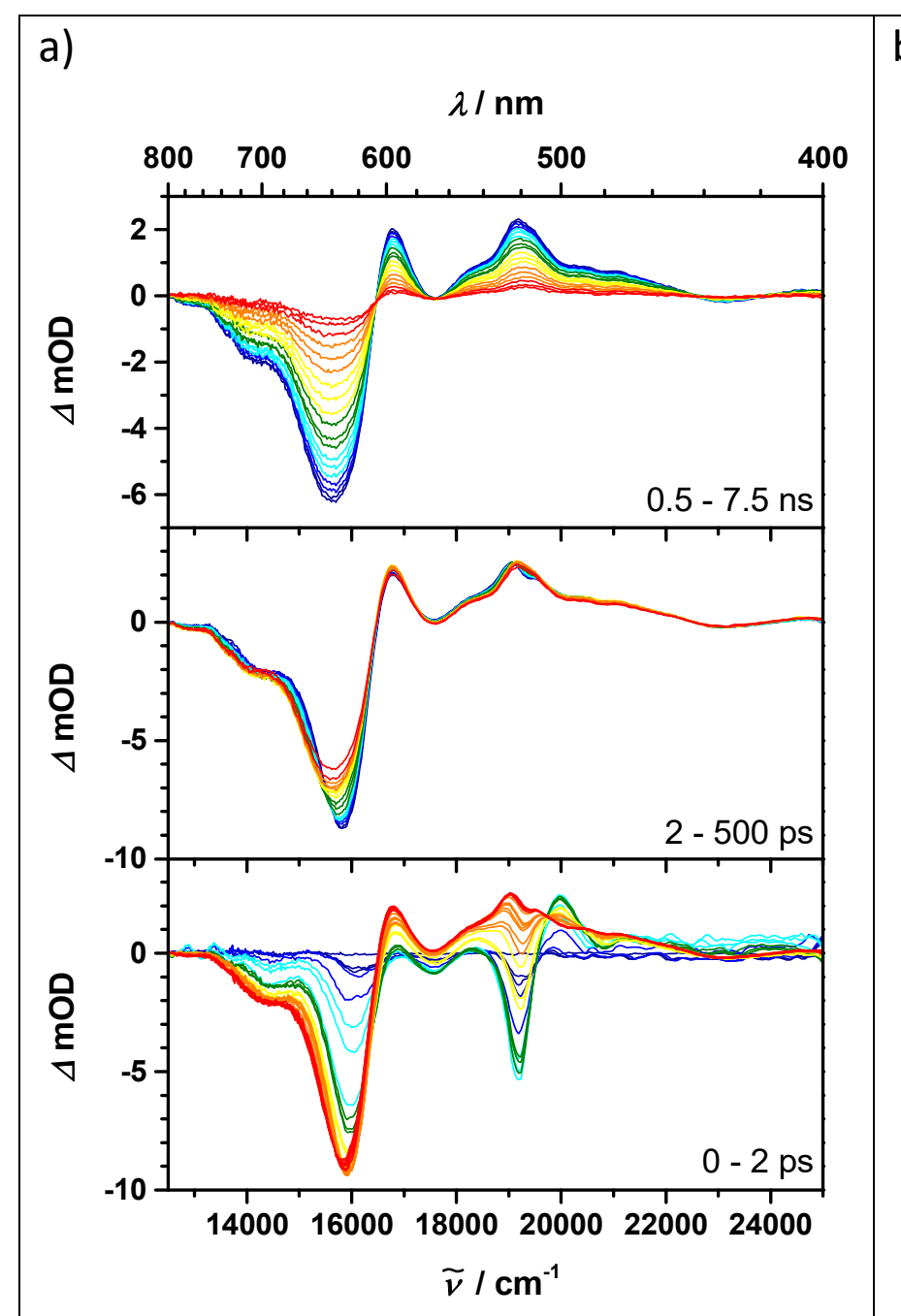

b)

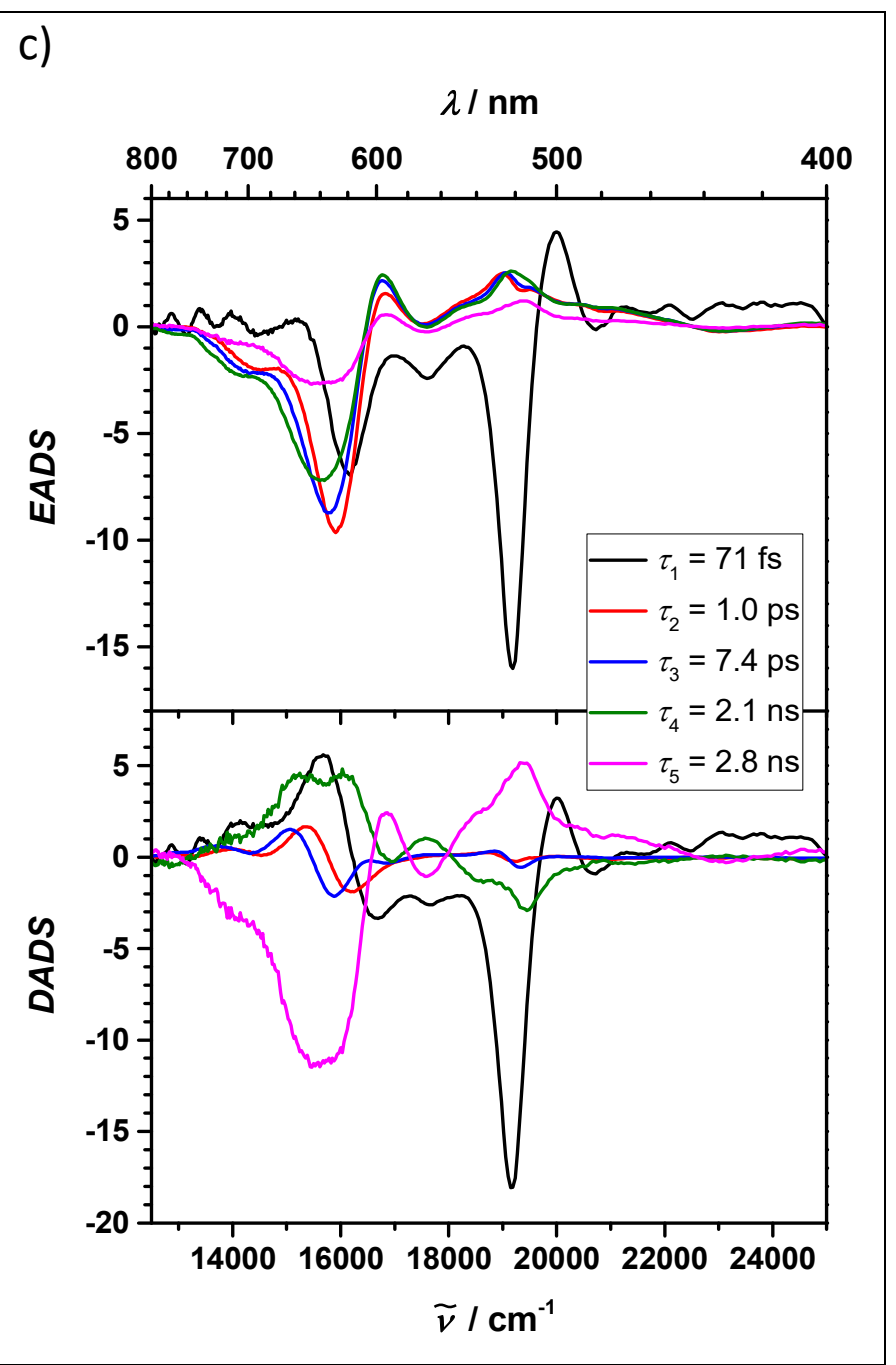

Figure S28. a) Transient spectra of 2G-Red in toluene excited at $485 \mathrm{~nm}$. Early spectra are given in blue, late spectra in red. b) Time scans and global fit at selected wavenumbers. c) Evolution associated difference spectra (top) and decay associated difference spectra (bottom) of a global fit using GLOTARAN software. 


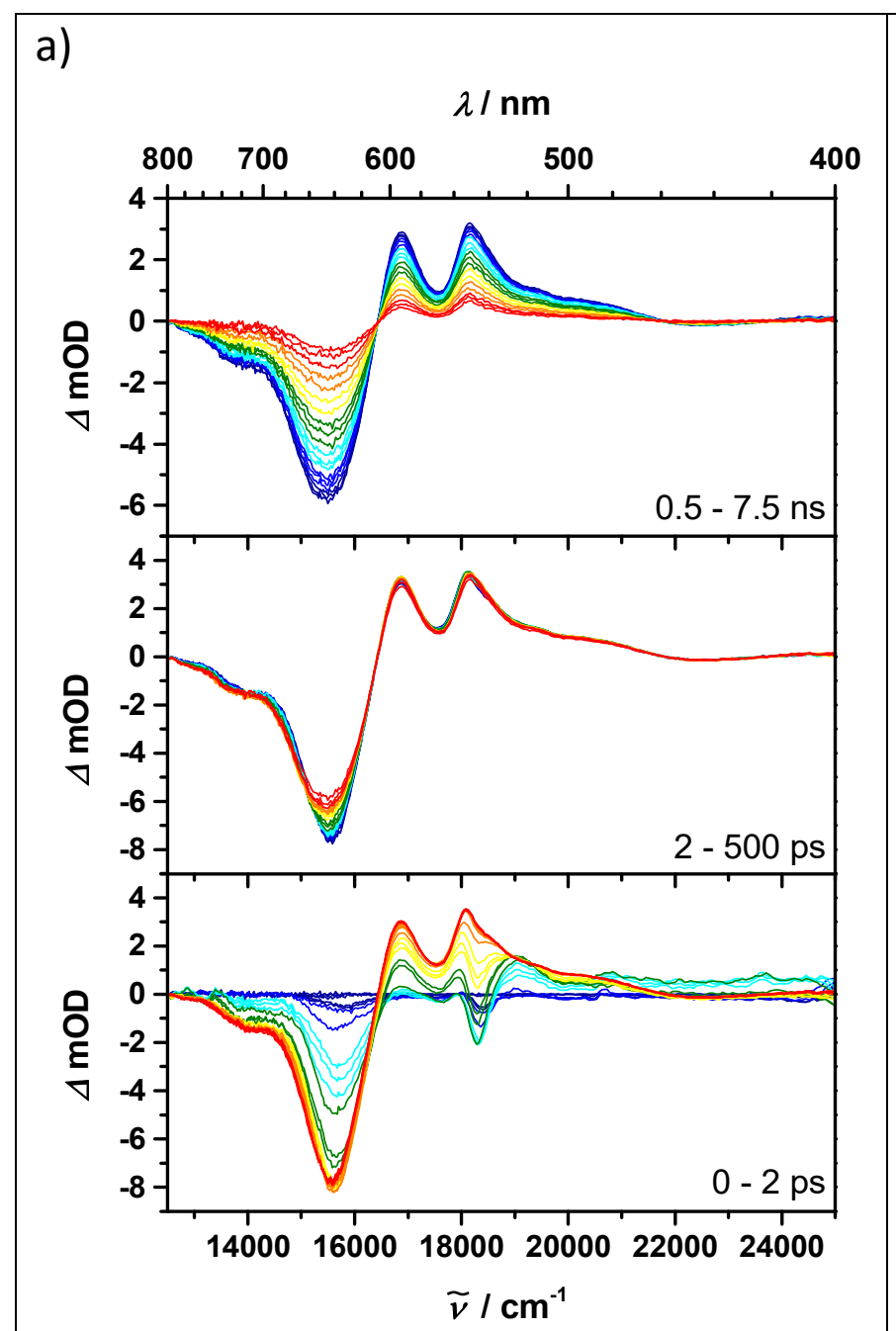

b)

400
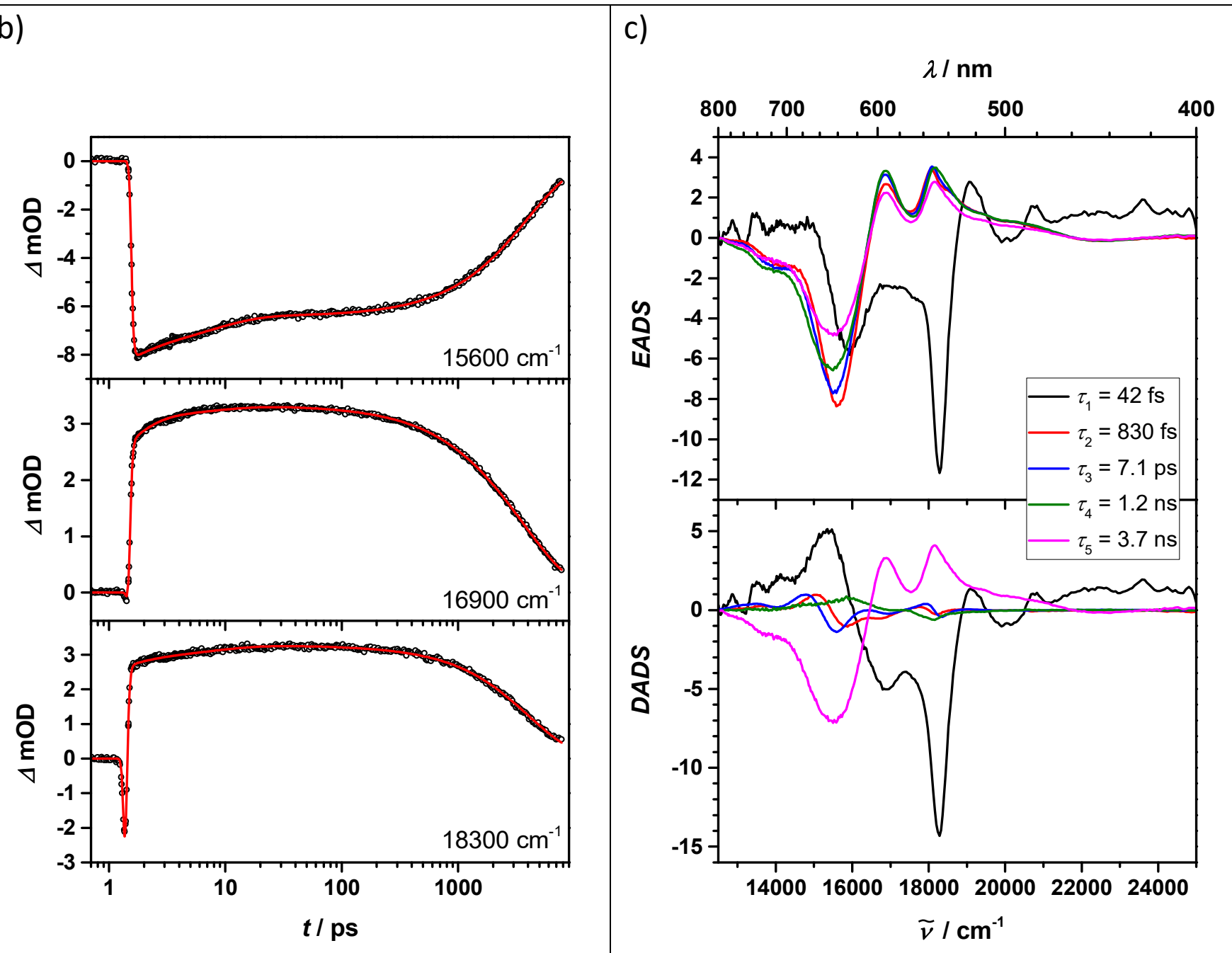

Figure S29. a) Transient spectra of 2G-Red| ${ }^{\mathrm{Et}}$ in toluene excited at $485 \mathrm{~nm}$. Early spectra are given in blue, late spectra in red. b) Time scans and global fit at selected wavenumbers. c) Evolution associated difference spectra (top) and decay associated difference spectra (bottom) of a global fit using GLOTARAN software. 

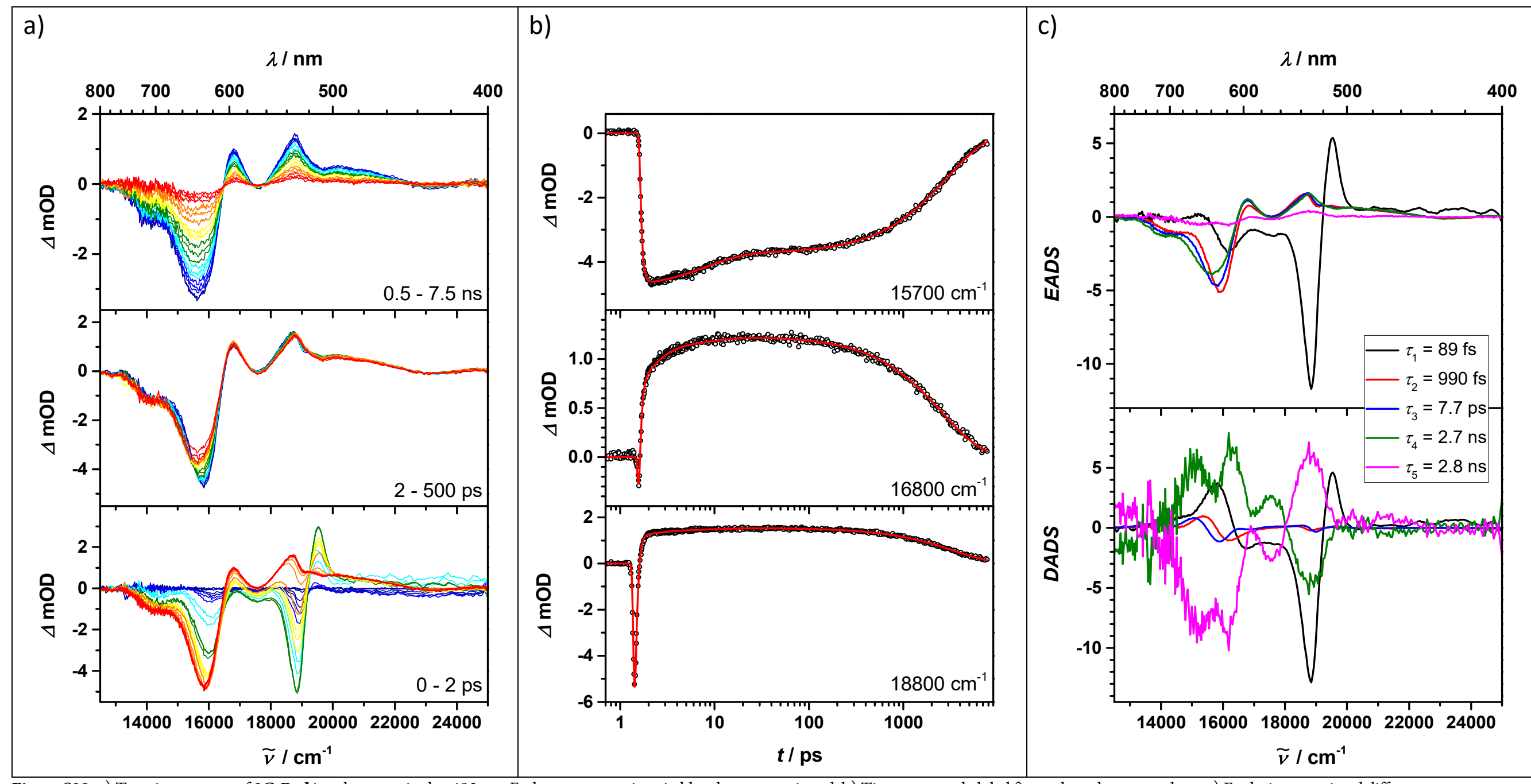

Figure S30. a) Transient spectra of 3G-Red in toluene excited at $485 \mathrm{~nm}$. Early spectra are given in blue, late spectra in red. b) Time scans and global fit at selected wavenumbers. c) Evolution associated difference spectra (top) and decay associated difference spectra (bottom) of a global fit using GLOTARAN software. 

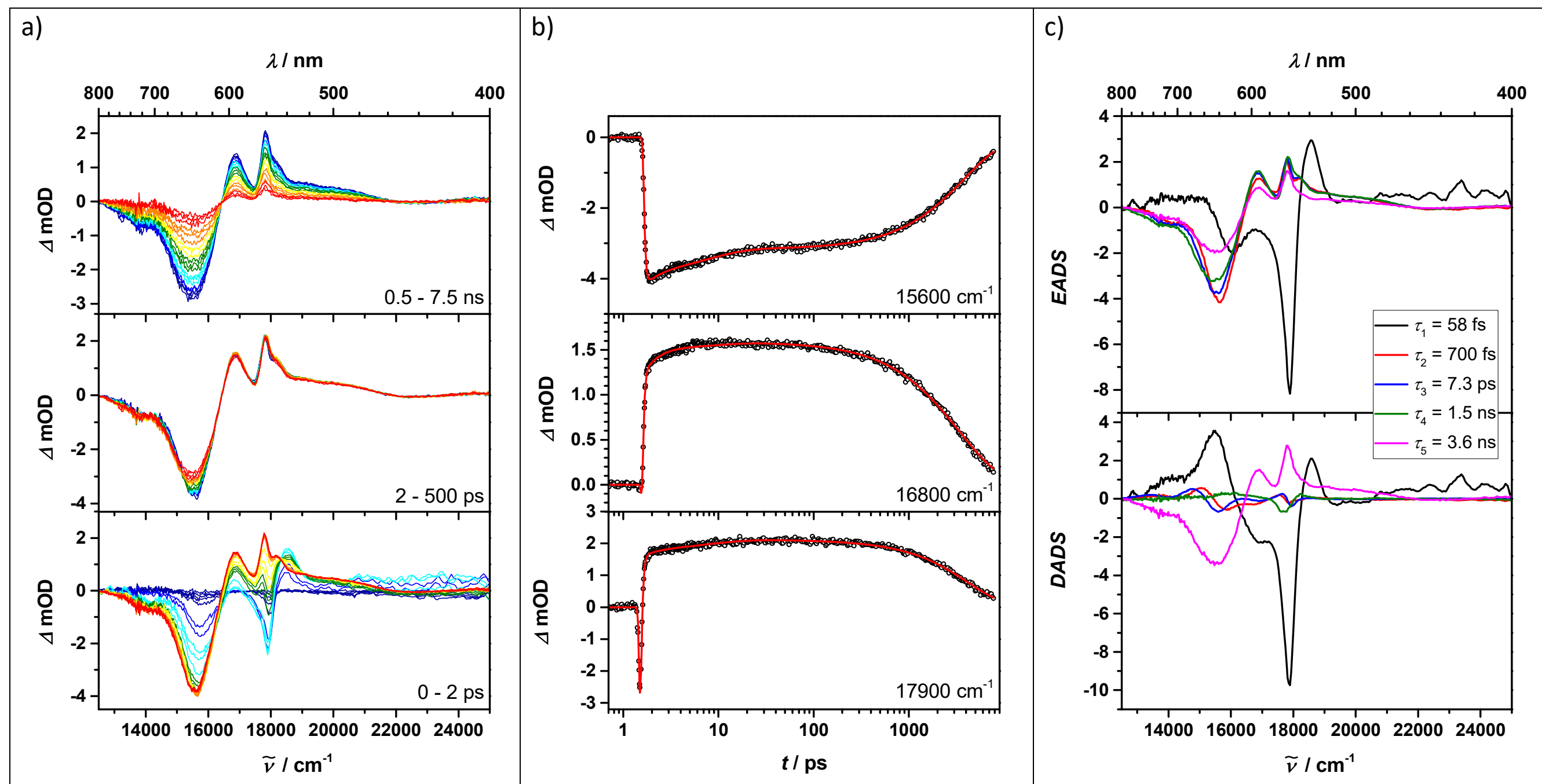

Figure S31. a) Transient spectra of 3G-Red $\left.\right|^{\text {Et }}$ in toluene excited at $485 \mathrm{~nm}$. Early spectra are given in blue, late spectra in red. b) Time scans and global fit at selected wavenumbers. c) Evolution associated difference spectra (top) and decay associated difference spectra (bottom) of a global fit using GLOTARAN software. 


\section{Computational Details}

\subsection{Computational archive for competing minimum superstructures of all heterooligomer}

\section{G-Red / saddle}

$\begin{array}{llll}3.18260500 & 0.44719000 & 2.14188600\end{array}$

C $\quad 2.01799600-0.03721600 \quad 1.58843100$

$\begin{array}{lllll}\text { C } & 2.37128400 & -0.98338000 & 0.60121200\end{array}$

$\begin{array}{lllll}\text { C } & 8.25886100 & -1.88698600 & -0.01958300\end{array}$

$\begin{array}{lll}7.89908700 & -0.94380500 & 0.93014900\end{array}$

$\begin{array}{llll}6.48907500 & -0.88777900 & 0.91037900\end{array}$

$5.60535900 \quad-0.12701300 \quad 1.65703800$

$\begin{array}{lll}4.24383800 & -0.22625700 & 1.48359800\end{array}$

$\begin{array}{lll}3.33046400 & 1.48151200 & 3.20524400\end{array}$

$\begin{array}{llll}3.83823600 & 2.36386400 & 2.81917600\end{array}$

$\begin{array}{lll}3.91095600 & 1.10444300 & 4.04480800\end{array}$

$\begin{array}{llll}2.35500100 & 1.78445100 & 3.57208300\end{array}$

$1.42912100-1.68752800-0.31026900$

$\begin{array}{llll}0.47122300 & -1.75092800 & 0.19369000\end{array}$

$\begin{array}{lll}1.78635700 & -2.69051300 & -0.52426400\end{array}$

$\begin{array}{llll}6.94541800 & -3.41659500 & -1.66150600\end{array}$

$6.38467600-4.27111800-1.28966700$

$\begin{array}{llll}7.92720800 & -3.74473500 & -1.98502900\end{array}$

$\begin{array}{llll}6.40043700 & -3.01558900 & -2.51292300\end{array}$

$\begin{array}{llll}8.80142600 & -0.12290600 & 1.78822500\end{array}$

$\begin{array}{llll}9.75819100 & -0.62032900 & 1.91643200\end{array}$

$\begin{array}{lll}8.36983200 & 0.04661200 & 2.77125900\end{array}$

$\begin{array}{lll}8.98829600 & 0.84884200 & 1.33338400\end{array}$

$\begin{array}{llll}3.69890300 & -1.09717700 & 0.54578000\end{array}$

$6.02389500-1.78434000-0.04008800$

$\begin{array}{llll}4.53028700 & -2.02244000 & -0.39046200\end{array}$

$\begin{array}{llll}4.18922100 & -3.35632900 & -0.17889800\end{array}$

$\begin{array}{llll}4.29740200 & -1.68708300 & -1.72698000\end{array}$

$\begin{array}{llll}2.12527000 & 2.64401100 & -1.31737600\end{array}$

$\begin{array}{llll}2.46188700 & 1.34255900 & -1.64738300\end{array}$

$\begin{array}{llll}1.31182200 & 0.54961200 & -1.49516000\end{array}$

$\begin{array}{llll}-3.26476100 & 2.21763800 & -0.08742100\end{array}$

$\begin{array}{llll}-3.58550500 & 3.56751800 & 0.22762900\end{array}$

$\begin{array}{llll}-2.44270600 & 4.31522400 & 0.09209600\end{array}$

$\begin{array}{llll}-1.41808800 & 3.41586600 & -0.31000100\end{array}$

$\begin{array}{llll}-0.08956700 & 3.64578300 & -0.58302500\end{array}$

$\begin{array}{llll}0.75631000 & 2.62015400 & -0.97343200\end{array}$

$\begin{array}{llll}3.00733400 & 3.84692300 & -1.33101400\end{array}$

$\begin{array}{llll}2.78878900 & 4.48296800 & -2.18745100\end{array}$

$\begin{array}{llll}2.87536700 & 4.44403500 & -0.43131500\end{array}$

$\begin{array}{llll}4.04918300 & 3.54735600 & -1.39227900\end{array}$

$\begin{array}{llll}1.23872100 & -0.92938300 & -1.65529800\end{array}$

$\begin{array}{llll}0.28345100 & -1.21930900 & -2.08301000\end{array}$

$\begin{array}{llll}2.03312500 & -1.22332900 & -2.33225000\end{array}$

$\begin{array}{llll}-4.11508700 & 1.05951300 & -0.08560000\end{array}$

$\begin{array}{llll}-2.27544200 & 5.78059700 & 0.31091200\end{array}$

$\begin{array}{llll}-3.21607400 & 6.22709000 & 0.61736000\end{array}$

$\begin{array}{llll}-1.53556200 & 5.97754500 & 1.08432700\end{array}$

$\begin{array}{llll}-1.94323100 & 6.27478000 & -0.59995300\end{array}$

$\begin{array}{llll}0.29022500 & 1.31877300 & -1.09487900\end{array}$

$\begin{array}{llll}-1.96396700 & 2.14627300 & -0.40954400\end{array}$

$\begin{array}{llll}-1.16376100 & 0.86722800 & -0.79456900\end{array}$

$\begin{array}{lll}-1.18147800 & -0.03571000 & 0.27426100\end{array}$

$\begin{array}{llll}-1.72622200 & 0.26206900 & -1.91693800\end{array}$

$\begin{array}{llll}5.99163000 & 0.56082100 & 2.39398500\end{array}$

$\begin{array}{llll}0.30150700 & 4.64796900 & -0.49417000\end{array}$

$\begin{array}{llll}-4.55349200 & 3.93217100 & 0.51844700\end{array}$

$\begin{array}{llll}1.00779400 & 0.24627600 & 1.82516700\end{array}$ $\begin{array}{llll}3.42499800 & 0.96988400 & -1.94888900\end{array}$ $\begin{array}{llll}9.25495100 & -2.19906600 & -0.27833800\end{array}$ $\begin{array}{llll}-5.41717200 & 1.11127400 & 0.24111000\end{array}$ $\begin{array}{lll}-5.84213900 & 2.06811700 & 0.51662500\end{array}$ $\begin{array}{lll}-3.65122400 & 0.12712800 & -0.36324000\end{array}$ $\begin{array}{lll}-6.34602100 & 0.00005900 & 0.26919000\end{array}$ $\begin{array}{llll}-7.67089400 & 0.22495100 & 0.64806500\end{array}$ $\begin{array}{llll}-5.99517900 & -1.31135300 & -0.07143900\end{array}$ $\begin{array}{lll}-8.60428000 & -0.78864200 & 0.69562600\end{array}$ $\begin{array}{llll}-7.97632200 & 1.22805600 & 0.91077000\end{array}$ $\begin{array}{llll}-6.90940600 & -2.33731600 & -0.03112400\end{array}$ $\begin{array}{llll}-4.98598300 & -1.53691500 & -0.37990500\end{array}$ $\begin{array}{lll}-8.24516800 & -2.10784900 & 0.36305800\end{array}$ $\begin{array}{lll}-9.61265200 & -0.55539400 & 0.99078400\end{array}$ $\begin{array}{llll}-6.58882200 & -3.32660500 & -0.30853900\end{array}$ $\begin{array}{llll}-9.14950800 & -3.12644500 & 0.41871000\end{array}$ $\begin{array}{llll}-8.78034200 & -4.44239600 & -0.05571600\end{array}$ $\begin{array}{llll}-7.94546400 & -4.84397600 & 0.51654000\end{array}$ $\begin{array}{llll}-9.62478600 & -5.10922600 & 0.06949300\end{array}$ $\begin{array}{llll}-8.50058200 & -4.43036500 & -1.11102800\end{array}$ $\begin{array}{lll}-10.53743900 & -2.83451600 & 0.70031000\end{array}$ $\begin{array}{llll}-11.09811200 & -3.76137200 & 0.70151200\end{array}$ $\begin{array}{lll}-10.64780700 & -2.37134800 & 1.67991200\end{array}$ $\begin{array}{llll}-10.97141500 & -2.16645000 & -0.04667100\end{array}$

Geometry: M052X-D3/def2-TZVPP/toluene (PCM) Frequency: M052X-D3/def2-SVP/toluene (PCM)

$\begin{array}{ll}\text { Zero-point correction= } & 0.677446 \\ \text { Thermal correction to Energy= } & 0.721700 \\ \text { Thermal correction to Enthalpy= } & 0.722644 \\ \text { Thermal correction to Gibbs Free Energy= } & 0.593318 \\ \text { Sum of electronic and zero-point Energies= } & -2079.213225 \\ \text { Sum of electronic and thermal Energies }= & -2079.168970 \\ \text { Sum of electronic and thermal Enthalpies }= & -2079.168026 \\ \text { Sum of electronic and thermal Free Energies }= & -2079.297353\end{array}$

Single Point: DSD-BLYP-D3/def2-TZVPP/toluene (SMD)

Dispersion correction $\quad-0.073053490$

FINAL SINGLE POINT ENERGY $\quad-2077.845071574010$

\section{G-Red / staggered}

$\begin{array}{llll}\text { C } & 2.38731500 & -2.90414800 & 0.42756000\end{array}$

$\begin{array}{lllll}\text { C } & 1.61985600 & -1.77239100 & 0.60820300\end{array}$

$\begin{array}{llll}\mathrm{C} & 2.48680500 & -0.65854800 & 0.59278000\end{array}$

$\begin{array}{lll}7.48837400 & -0.80272600 & -0.10718000\end{array}$

$\begin{array}{llll}8.25675200 & -1.96304900 & -0.31409600\end{array}$

$\begin{array}{llll}7.40237700 & -3.05132700 & -0.26933600\end{array}$

$\begin{array}{llll}6.11202000 & -2.52655800 & -0.03470900\end{array}$

$\begin{array}{llll}4.89049400 & -3.16372800 & 0.08588100\end{array}$

$\begin{array}{llll}3.72705800 & -2.45755400 & 0.30004800\end{array}$

$\begin{array}{llll}1.93103300 & -4.32273800 & 0.37618800\end{array}$

$\begin{array}{llll}2.35868500 & -4.90000100 & 1.19380500\end{array}$

$\begin{array}{llll}2.22958000 & -4.79647800 & -0.55686700\end{array}$

$\begin{array}{llll}0.84967900 & -4.37319000 & 0.45360000\end{array}$ 
$\begin{array}{llll}2.09936800 & 0.77225000 & 0.69049300\end{array}$ $\begin{array}{llll}2.92757200 & 1.36664100 & 1.06400500\end{array}$ $\begin{array}{llll}1.26063600 & 0.86280700 & 1.37489200\end{array}$ $\begin{array}{llll}7.95068300 & 0.60940000 & -0.07791700\end{array}$ $\begin{array}{llll}7.55521700 & 1.15288000 & -0.93365000\end{array}$ $\begin{array}{llll}9.03441600 & 0.64492100 & -0.10425000\end{array}$ $\begin{array}{llll}7.59261800 & 1.10829100 & 0.81909300\end{array}$ $\begin{array}{llll}7.75022900 & -4.49417900 & -0.41685300\end{array}$ $\begin{array}{llll}8.76232300 & -4.60208700 & -0.79421600\end{array}$ $\begin{array}{llll}7.07354300 & -4.99547300 & -1.10511400\end{array}$ $\begin{array}{llll}7.69073500 & -5.00773400 & 0.54137900\end{array}$ $\begin{array}{llll}3.74085700 & -1.07203600 & 0.40765400\end{array}$ $\begin{array}{llll}6.20739000 & -1.14528700 & 0.05933100\end{array}$ $\begin{array}{llll}5.02021500 & -0.18636000 & 0.34936700\end{array}$ $\begin{array}{llll}4.90263100 & 0.76185100 & -0.66715100\end{array}$ $\begin{array}{llll}5.21337300 & 0.46007600 & 1.56959000\end{array}$ $\begin{array}{llll}1.01090200 & 4.93233700 & -0.36003700\end{array}$ $\begin{array}{llll}1.89364500 & 3.88499800 & -0.57163400\end{array}$ $\begin{array}{llll}1.15051900 & 2.69345700 & -0.57659100\end{array}$ $\begin{array}{llll}-3.85341700 & 2.31162800 & 0.10959000\end{array}$ $\begin{array}{llll}-4.73421100 & 3.40105700 & 0.35781800\end{array}$ $\begin{array}{llll}-3.98987600 & 4.55370000 & 0.35570200\end{array}$ $\begin{array}{llll}-2.64519600 & 4.16508100 & 0.10566900\end{array}$ $\begin{array}{lll}-1.50524100 & 4.92768300 & 0.00341300\end{array}$ $\begin{array}{llll}-0.26832300 & 4.34901100 & -0.23595300\end{array}$ $\begin{array}{llll}1.32190300 & 6.38986000 & -0.29728700\end{array}$ $\begin{array}{llll}1.06487200 & 6.88226900 & -1.23400600\end{array}$ $\begin{array}{llll}0.76708300 & 6.87990400 & 0.49929000\end{array}$ $\begin{array}{llll}2.38187600 & 6.54242100 & -0.11845600\end{array}$ $\begin{array}{llll}1.66256400 & 1.30274000 & -0.69484800\end{array}$ $\begin{array}{llll}0.89148200 & 0.65582200 & -1.10295300\end{array}$ $\begin{array}{llll}2.52188100 & 1.28636000 & -1.35931400\end{array}$ $\begin{array}{llll}-4.15075000 & 0.90942900 & 0.00743100\end{array}$ $\begin{array}{llll}-4.46397600 & 5.95070800 & 0.57085800\end{array}$ $\begin{array}{llll}-5.53971000 & 5.96716500 & 0.71439500\end{array}$ $\begin{array}{llll}-3.99511300 & 6.38976300 & 1.44937200\end{array}$ $\begin{array}{llll}-4.22294800 & 6.57998500 & -0.28353400\end{array}$ $\begin{array}{llll}-0.14386500 & 2.97518800 & -0.37573000\end{array}$ $\begin{array}{llll}-2.60746600 & 2.78690900 & -0.03631300\end{array}$ $\begin{array}{llll}-1.32137800 & 1.96433400 & -0.34008000\end{array}$ $\begin{array}{llll}-1.11035200 & 1.00893700 & 0.65842300\end{array}$ $\begin{array}{llll}-1.44804600 & 1.31881600 & -1.57060400\end{array}$ $\begin{array}{llll}4.84654700 & -4.23934200 & 0.00310600\end{array}$ $\begin{array}{llll}-1.57590100 & 5.99860400 & 0.11797900\end{array}$ $\begin{array}{llll}-5.38779400 & 0.41214400 & 0.17090200\end{array}$ $\begin{array}{llll}-6.19409000 & 1.09833900 & 0.39695600\end{array}$ $\begin{array}{llll}-3.31727200 & 0.26099800 & -0.20870400\end{array}$ $\begin{array}{llll}-5.77842000 & -0.98016400 & 0.08064000\end{array}$ $\begin{array}{llll}-7.10721500 & -1.33494500 & 0.32077400\end{array}$ $\begin{array}{llll}-4.89280100 & -2.01365900 & -0.24460700\end{array}$ $\begin{array}{llll}-7.54348800 & -2.64109600 & 0.25285700\end{array}$ $\begin{array}{llll}-7.81872600 & -0.55941800 & 0.56725400\end{array}$ $\begin{array}{llll}-5.30624600 & -3.32293700 & -0.31874700\end{array}$ $\begin{array}{llll}-3.85754400 & -1.79105400 & -0.45254900\end{array}$ $\begin{array}{llll}-6.64770500 & -3.67909000 & -0.06250600\end{array}$ $\begin{array}{llll}-8.58086300 & -2.85448000 & 0.44420100\end{array}$ $\begin{array}{llll}-4.58569000 & -4.07836100 & -0.58071200\end{array}$ $\begin{array}{llll}-7.05690700 & -4.97876300 & -0.11980000\end{array}$ $\begin{array}{llll}-6.14319700 & -5.99949200 & -0.58447500\end{array}$ $\begin{array}{llll}-5.26893900 & -6.06440700 & 0.06146700\end{array}$ $\begin{array}{llll}-6.64814500 & -6.95761000 & -0.56254500\end{array}$ $\begin{array}{llll}-5.80434800 & -5.80843500 & -1.60478200\end{array}$ $\begin{array}{llll}-8.46282400 & -5.29062300 & 0.01234500\end{array}$ $\begin{array}{llll}-8.59288500 & -6.36347000 & -0.06185200\end{array}$ $\begin{array}{llll}-8.84393400 & -4.97049800 & 0.98102100\end{array}$ $\begin{array}{llll}-9.05999600 & -4.81291200 & -0.76734300\end{array}$ $\begin{array}{llll}9.31942700 & -1.98019300 & -0.47810400\end{array}$ $\begin{array}{llll}0.55341500 & -1.71729500 & 0.73863200\end{array}$ $\begin{array}{llll}2.95916300 & 3.94822100 & -0.70521700\end{array}$ $\begin{array}{llll}-5.79466000 & 3.32880300 & 0.51473200\end{array}$

\section{Geometry: M052X-D3/def2-TZVPP/toluene (PCM)} Frequency: M052X-D3/def2-SVP/toluene (PCM)

$\begin{array}{ll}\text { Zero-point correction= } & 0.677392 \\ \text { Thermal correction to Energy= } & 0.721755 \\ \text { Thermal correction to Enthalpy= } & 0.722699 \\ \text { Thermal correction to Gibbs Free Energy= } & 0.592150 \\ \text { Sum of electronic and zero-point Energies= } & -2079.212210 \\ \text { Sum of electronic and thermal Energies= } & -2079.167847 \\ \text { Sum of electronic and thermal Enthalpies }= & -2079.166903 \\ \text { Sum of electronic and thermal Free Energies }= & -2079.297452\end{array}$

Single Point: DSD-BLYP-D3/def2-TZVPP/toluene (SMD)

Dispersion correction $\quad-0.070615381$

FINAL SINGLE POINT ENERGY $\quad-2077.843140548619$

\section{G-Red $\left.\right|^{\mathrm{Et}} /$ saddle}

$\begin{array}{lll}-3.57819600 & -1.21802300 \quad 2.20203700\end{array}$ $\begin{array}{lll}-2.28085700 & -0.93004800 & 1.81237500\end{array}$ $\begin{array}{lll}-2.34846300 & 0.18506100 & 0.94748200\end{array}$ $\begin{array}{lll}-6.55636900 & 2.62092300 & -0.45652700\end{array}$ $\begin{array}{lll}-7.87531500 & 2.30784000 & -0.06255500\end{array}$ $\begin{array}{llll}-7.79470700 & 1.21823600 & 0.79224900\end{array}$ $\begin{array}{lll}-6.42417900 & 0.89134600 & 0.90519400\end{array}$ $\begin{array}{lll}-5.78664100 & -0.10976300 & 1.61255800\end{array}$ $\begin{array}{lll}-4.41585200 & -0.26815700 & 1.57059100\end{array}$ $\begin{array}{lll}-4.00975000 & -2.32821200 & 3.09805300\end{array}$ $\begin{array}{lll}-5.07586900 & -2.29156900 & 3.29949200\end{array}$ $\begin{array}{lll}-3.48294600 & -2.28311400 & 4.04918200\end{array}$ $\begin{array}{lll}-3.78383400 & -3.29315800 & 2.64567200\end{array}$ $\begin{array}{llll}-1.21661900 & 0.82358900 & 0.22246500\end{array}$ $\begin{array}{lll}-0.31094200 & 0.71108500 & 0.81007000\end{array}$ $\begin{array}{lll}-1.41660600 & 1.88362100 & 0.09383400\end{array}$ $\begin{array}{llll}-6.11290600 & 3.69217400 & -1.38626900\end{array}$ $\begin{array}{llll}-5.25761000 & 4.22306700 & -0.97790100\end{array}$ $\begin{array}{llll}-6.92165500 & 4.39261300 & -1.56690800\end{array}$ $\begin{array}{llll}-5.80618900 & 3.25907700 & -2.33703300\end{array}$ $\begin{array}{lll}-8.93090600 & 0.52603400 & 1.46512400\end{array}$ $\begin{array}{lll}-9.45265500 & 1.20627200 & 2.13634800\end{array}$ $\begin{array}{lll}-8.59196000 & -0.32543000 & 2.04702700\end{array}$ $\begin{array}{llll}-9.65398100 & 0.16963600 & 0.73365600\end{array}$ $\begin{array}{llll}-3.62156300 & 0.57882800 & 0.81109400\end{array}$ $\begin{array}{llll}-5.69781700 & 1.77764000 & 0.12609400\end{array}$ $\begin{array}{llll}-4.15310100 & 1.77574800 & -0.02726100\end{array}$ $\begin{array}{llll}-3.61797300 & 2.97236800 & 0.44928500\end{array}$ $\begin{array}{llll}-3.81330600 & 1.61715500 & -1.37329500\end{array}$ $\begin{array}{llll}-0.97057800 & -3.48850800 & -1.07915700\end{array}$ $\begin{array}{lll}-1.65963300 & -2.29261500 & -1.22529900\end{array}$ $\begin{array}{lll}-0.70803800 & -1.25578600 & -1.14109400\end{array}$ $\begin{array}{llll}4.28526900 & -1.82496100 & -0.40280300\end{array}$ $\begin{array}{llll}4.96462400 & -3.07189300 & -0.25336200\end{array}$ $\begin{array}{llll}4.01127400 & -4.06414200 & -0.35891700\end{array}$ $\begin{array}{llll}2.76403900 & -3.42616900 & -0.57212700\end{array}$ $\begin{array}{llll}1.50202200 & -3.96024700 & -0.72254400\end{array}$ $\begin{array}{llll}1.39169800 & -3.15572900 & -0.91321800\end{array}$ $\begin{array}{llll}0.55904500 & -4.85819400 & -1.08014000\end{array}$ $\begin{array}{llll}-2.10349400 & -5.04564500 & -2.00381700\end{array}$ $\begin{array}{llll}-0.79542000 & -5.62304600 & -0.97715400\end{array}$ $\begin{array}{llll}-2.26424200 & -4.97217000 & -0.25764100\end{array}$ $\begin{array}{llll}-0.97356700 & 0.20875400 & -1.18019600\end{array}$ 

$4.77680600-0.47147200-0.39555600$ $\begin{array}{llll}4.24848500 & -5.53483700 & -0.28952400\end{array}$ $\begin{array}{llll}4.85541100 & -5.86618500 & -1.13089800\end{array}$ $\begin{array}{llll}4.77948400 & -5.80020100 & 0.62225100\end{array}$ $\begin{array}{llll}3.31615300 & -6.09025300 & -0.31070200\end{array}$ $\begin{array}{llll}0.51588700 & -1.77699700 & -0.95979500\end{array}$ $\begin{array}{llll}2.97305300 & -2.06153800 & -0.60202600\end{array}$ $\begin{array}{llll}1.85395000 & -1.00251900 & -0.81656900\end{array}$ $\begin{array}{llll}1.79501000 & -0.14085300 & 0.28271800\end{array}$ $\begin{array}{llll}2.10556400 & -0.25945300 & -1.97188000\end{array}$ $\begin{array}{llll}-6.37490900 & -0.78912900 & 2.20872100\end{array}$ $\begin{array}{llll}1.37548300 & -5.03027000 & -0.68591000\end{array}$ $\begin{array}{llll}5.99470400 & -0.07855100 & 0.01345400\end{array}$ $\begin{array}{llll}6.69050200 & -0.81090900 & 0.39166700\end{array}$ $\begin{array}{llll}4.06826000 & 0.26857800 & -0.73064900\end{array}$ $\begin{array}{llll}6.48787200 & 1.28652000 & 0.03328300\end{array}$ $\begin{array}{llll}7.76400300 & 1.54070300 & 0.53862100\end{array}$ $\begin{array}{llll}5.75695500 & 2.38635700 & -0.42865100\end{array}$ $\begin{array}{llll}8.29367800 & 2.81295000 & 0.59583400\end{array}$ $\begin{array}{llll}8.35861600 & 0.71168600 & 0.89671900\end{array}$ $\begin{array}{llll}6.26544200 & 3.66343300 & -0.38093200\end{array}$ $\begin{array}{llll}4.76893400 & 2.24519200 & -0.83894100\end{array}$ $\begin{array}{llll}7.55000200 & 3.91782700 & 0.14412400\end{array}$ $\begin{array}{llll}9.28551000 & 2.94720100 & 0.99165900\end{array}$ $\begin{array}{llll}5.66220900 & 4.47319500 & -0.75359400\end{array}$ $\begin{array}{llll}8.04790300 & 5.18739000 & 0.21085700\end{array}$ $\begin{array}{llll}7.32426600 & 6.27336400 & -0.41371300\end{array}$ $\begin{array}{llll}6.34053700 & 6.39626900 & 0.03620800\end{array}$ $\begin{array}{llll}7.87738500 & 7.19306300 & -0.26573400\end{array}$ $\begin{array}{llll}7.19489600 & 6.11375600 & -1.48640400\end{array}$ $\begin{array}{llll}9.42216600 & 5.39188700 & 0.61085600\end{array}$ $\begin{array}{llll}9.63415500 & 6.45429900 & 0.60813000\end{array}$ $\begin{array}{llll}9.59346300 & 5.01695400 & 1.61868600\end{array}$ $\begin{array}{llll}10.12236900 & 4.89479900 & -0.06460100\end{array}$ $\begin{array}{llll}6.42255200 & -3.31763400 & -0.02857000\end{array}$ $\begin{array}{llll}7.01511900 & -2.59266100 & -0.58231700\end{array}$ $\begin{array}{llll}6.67217900 & -4.29395000 & -0.44171900\end{array}$ $\begin{array}{llll}-3.12485500 & -2.13087200 & -1.47653600\end{array}$ $\begin{array}{llll}-3.65854600 & -2.95459200 & -1.00281700\end{array}$ $\begin{array}{llll}-3.48450600 & -1.21684800 & -1.00994700\end{array}$ $\begin{array}{llll}-3.45119000 & -2.09697800 & -2.97144900\end{array}$ $\begin{array}{llll}-3.11290600 & -3.00850100 & -3.46018400\end{array}$ $\begin{array}{llll}-4.52163800 & -1.99240200 & -3.13281600\end{array}$ $\begin{array}{llll}-2.95064300 & -1.25677400 & -3.44793000\end{array}$ $\begin{array}{llll}-1.03552900 & -1.62619400 & 2.25991100\end{array}$ $\begin{array}{llll}-1.27164500 & -2.66667900 & 2.48126400\end{array}$ $\begin{array}{lll}-0.30410800 & -1.63605100 & 1.45549400\end{array}$ $\begin{array}{lll}-0.42013500 & -0.96225700 & 3.49396000\end{array}$ $\begin{array}{llll}-1.12737900 & -0.95608700 & 4.32104500\end{array}$ $\begin{array}{llll}0.47906700 & -1.48683000 & 3.80812600\end{array}$ $\begin{array}{lll}-0.15298500 & 0.06920100 & 3.27459700\end{array}$ $\begin{array}{llll}6.80078000 & -3.29868000 & 1.45574800\end{array}$ $\begin{array}{llll}6.26965300 & -4.08518800 & 1.98728100\end{array}$ $\begin{array}{llll}7.86897100 & -3.45581000 & 1.58789200\end{array}$ $\begin{array}{lll}6.52940400 & -2.35319900 & 1.91961500\end{array}$ $\begin{array}{llll}-9.11818700 & 2.99381400 & -0.53114400\end{array}$ $\begin{array}{llll}-8.96100000 & 4.07146400 & -0.55512000\end{array}$ $\begin{array}{llll}-9.91551400 & 2.81354100 & 0.18803000\end{array}$ $\begin{array}{lll}-9.55696700 & 2.51013500 & -1.91518400\end{array}$ $\begin{array}{lll}-10.46358300 & 3.01651600 & -2.23850400\end{array}$ $\begin{array}{llll}-8.77676400 & 2.69933300 & -2.64962300\end{array}$ $\begin{array}{llll}-9.74543700 & 1.43871800 & -1.89980900\end{array}$

Geometry: M052X-D3/def2-TZVPP/toluene (PCM) Frequency: M052X-D3/def2-SVP/toluene (PCM)

$\begin{array}{ll}\text { Zero-point correction= } & 0.906575 \\ \text { Thermal correction to Energy= } & 0.962626 \\ \text { Thermal correction to Enthalpy= } & 0.963570 \\ \text { Thermal correction to Gibbs Free Energy= } & 0.809240 \\ \text { Sum of electronic and zero-point Energies= } & -2393.584930 \\ \text { Sum of electronic and thermal Energies }= & -2393.528879 \\ \text { Sum of electronic and thermal Enthalpies }= & -2393.527935 \\ \text { Sum of electronic and thermal Free Energies }= & -2393.682265\end{array}$

Single Point: DSD-BLYP-D3/def2-TZVPP/toluene (SMD)

Dispersion correction $\quad-0.093499900$

FINAL SINGLE POINT ENERGY $\quad-2392.100174591758$

\section{G-Red $\left.\right|^{\text {Et }} /$ staggered}

$\begin{array}{lllll}\text { C } & -1.31263500 & 1.94009100 & 0.18792200\end{array}$

$\begin{array}{lllll}\text { C } & -2.04344200 & 0.75925900 & 0.45600000\end{array}$

$\begin{array}{lllll}\text { C } & -7.07615900 & 0.28666100 & 0.46791100\end{array}$

$\begin{array}{lllll}\text { C } & -8.01254900 & 1.31586900 & 0.23667500\end{array}$

$\begin{array}{lllll}\text { C } & -7.28920600 & 2.48172300 & 0.02534800\end{array}$

$\begin{array}{lllll}\text { C } & -5.92366900 & 2.13817700 & 0.13677200\end{array}$

$\begin{array}{lll}-4.78167000 & 2.90616500 & -0.00325600\end{array}$

$\begin{array}{lll}-3.52147800 & 2.36018800 & 0.12162200\end{array}$

$\begin{array}{llll}-1.94764400 & 4.37525500 & -0.33038800\end{array}$

$\begin{array}{llll}-2.85526000 & 4.96858900 & -0.38289600\end{array}$

$\begin{array}{llll}-1.43298900 & 4.45912200 & -1.28633900\end{array}$

$\begin{array}{lll}-1.30099000 & 4.81153600 & 0.42850700\end{array}$

$\begin{array}{lll}-1.48549100 & -0.59811200 & 0.69819600\end{array}$

$\begin{array}{lll}-2.18582900 & -1.19465900 & 1.27522300\end{array}$

$\begin{array}{lll}-0.55922900 & -0.50929000 & 1.25799600\end{array}$

$\begin{array}{lll}-7.34780600 & -1.15326100 & 0.71766800\end{array}$

$\begin{array}{llll}-7.13727200 & -1.73544800 & -0.17840600\end{array}$

$\begin{array}{llll}-8.38895000 & -1.29781200 & 0.98759500\end{array}$

$\begin{array}{llll}-6.71138400 & -1.53161200 & 1.51233400\end{array}$

$\begin{array}{llll}-7.84401100 & 3.83529000 & -0.26296400\end{array}$

$\begin{array}{llll}-8.47829900 & 3.81252100 & -1.14731300\end{array}$

$\begin{array}{llll}-7.05583100 & 4.56243200 & -0.43200100\end{array}$

$\begin{array}{llll}-8.45367000 & 4.18665500 & 0.56793400\end{array}$

$\begin{array}{llll}-3.35485800 & 1.01273200 & 0.40918300\end{array}$

$\begin{array}{llll}-5.83509800 & 0.78306000 & 0.41197900\end{array}$

$\begin{array}{llll}-4.51366800 & 0.00072900 & 0.62645000\end{array}$

$\begin{array}{llll}-4.42132000 & -1.04414600 & -0.29858600\end{array}$

$\begin{array}{llll}-4.46464000 & -0.52401400 & 1.91906500\end{array}$

$\begin{array}{llll}-0.27477100 & -4.84312300 & -0.08874500\end{array}$

$\begin{array}{llll}-1.24463700 & -3.86859600 & -0.27482100\end{array}$

$\begin{array}{llll}-0.56880300 & -2.64136600 & -0.43314900\end{array}$

$\begin{array}{llll}4.44091400 & -1.91158200 & -0.18558800\end{array}$

$\begin{array}{llll}5.42186200 & -2.92853400 & -0.00123800\end{array}$

$\begin{array}{llll}4.74482300 & -4.13055400 & 0.08053200\end{array}$

$\begin{array}{llll}3.36430500 & -3.84366000 & -0.05424700\end{array}$

$\begin{array}{llll}2.26330900 & -4.67615500 & 0.00065300\end{array}$

$\begin{array}{llll}0.97667400 & -4.18527200 & -0.12775900\end{array}$

$\begin{array}{llll}-0.50497200 & -6.30138000 & 0.11756700\end{array}$

$\begin{array}{llll}-1.02517400 & -6.73551500 & -0.73450000\end{array}$

$\begin{array}{llll}0.42875400 & -6.83857400 & 0.25277700\end{array}$

$\begin{array}{llll}-1.12250200 & -6.47109200 & 0.99801800\end{array}$

$\begin{array}{llll}-1.18376400 & -1.30276800 & -0.64455600\end{array}$

$\begin{array}{llll}-0.51868100 & -0.68247400 & -1.23834400\end{array}$

$\begin{array}{llll}-2.11852800 & -1.42063000 & -1.18596400\end{array}$

$\begin{array}{llll}4.55980000 & -0.48296800 & -0.32812600\end{array}$ 

$\begin{array}{lll}6.01092800 & -5.50235100 & 1.12063900\end{array}$ $\begin{array}{llll}4.59358000 & -6.24526200 & 0.38899900\end{array}$ $\begin{array}{llll}0.75367700 & -2.83362300 & -0.33656700\end{array}$ $\begin{array}{llll}3.22034400 & -2.48259600 & -0.22952100\end{array}$ $\begin{array}{llll}1.86188900 & -1.75277200 & -0.39297900\end{array}$ $\begin{array}{llll}1.68956700 & -0.83307800 & 0.64738100\end{array}$ $1.81432800-1.07300800-1.61487100$ $\begin{array}{llll}-4.87905100 & 3.95687000 & -0.22587000\end{array}$ $\begin{array}{llll}2.40998200 & -5.73312100 & 0.15504900\end{array}$ $\begin{array}{llll}5.64368800 & 0.25988700 & -0.05411000\end{array}$ $\begin{array}{llll}6.54266500 & -0.21493000 & 0.30755800\end{array}$ $\begin{array}{llll}3.65809400 & 0.01017500 & -0.65212600\end{array}$ $\begin{array}{llll}5.72112800 & 1.70710600 & -0.16215100\end{array}$ $\begin{array}{llll}6.90356500 & 2.35536400 & 0.19646400\end{array}$ $\begin{array}{llll}4.66066600 & 2.51343800 & -0.58914000\end{array}$ $\begin{array}{llll}7.03937300 & 3.72720800 & 0.13691000\end{array}$ $\begin{array}{llll}7.74053800 & 1.76302800 & 0.53924700\end{array}$ $\begin{array}{llll}4.77416000 & 3.88301800 & -0.65563200\end{array}$ $\begin{array}{llll}3.72026600 & 2.06624300 & -0.87364500\end{array}$ $\begin{array}{llll}5.97446500 & 4.53341200 & -0.30153400\end{array}$ $\begin{array}{llll}7.97406100 & 4.17041200 & 0.43399500\end{array}$ $\begin{array}{llll}3.92421900 & 4.45512100 & -0.98596600\end{array}$ $\begin{array}{llll}6.09458100 & 5.89279300 & -0.38488100\end{array}$ $\begin{array}{llll}4.91888300 & 6.69548400 & -0.64296500\end{array}$ $\begin{array}{llll}4.14907600 & 6.55052700 & 0.11873100\end{array}$ $\begin{array}{llll}5.20425600 & 7.74063100 & -0.65190600\end{array}$ $\begin{array}{llll}4.49016300 & 6.45669500 & -1.61454700\end{array}$ $\begin{array}{llll}7.27288700 & 6.53301300 & 0.15673300\end{array}$ $\begin{array}{llll}7.19299200 & 7.60241800 & 0.00236900\end{array}$ $\begin{array}{llll}7.38832900 & 6.34162200 & 1.22641300\end{array}$ $\begin{array}{llll}8.17038100 & 6.18682600 & -0.35263000\end{array}$ $\begin{array}{llll}6.90614000 & -2.77532600 & 0.09421300\end{array}$ $\begin{array}{llll}7.24214000 & -1.97722500 & -0.56451800\end{array}$ $\begin{array}{llll}7.37092200 & -3.68989300 & -0.27176500\end{array}$ $\begin{array}{llll}-2.72609100 & -4.06084700 & -0.34024400\end{array}$ $\begin{array}{llll}-2.99971300 & -4.91921300 & 0.27202500\end{array}$ $\begin{array}{llll}-3.23065800 & -3.19489200 & 0.08347500\end{array}$ $\begin{array}{llll}-3.22008700 & -4.27673500 & -1.77285000\end{array}$ $\begin{array}{llll}-2.74741700 & -5.15169900 & -2.21471000\end{array}$ $\begin{array}{llll}-4.29844200 & -4.41637600 & -1.79354700\end{array}$ $\begin{array}{llll}-2.97666700 & -3.41638900 & -2.39245200\end{array}$ $\begin{array}{llll}0.17807300 & 2.06324500 & 0.16067100\end{array}$ $\begin{array}{llll}0.61720000 & 1.16247600 & -0.26181300\end{array}$ $\begin{array}{llll}0.44976000 & 2.88833000 & -0.49744000\end{array}$ $\begin{array}{llll}0.77471800 & 2.29965000 & 1.55006100\end{array}$ $\begin{array}{llll}0.53366600 & 1.47222700 & 2.21291400\end{array}$ $\begin{array}{llll}1.85824900 & 2.38136500 & 1.49499400\end{array}$ $\begin{array}{llll}0.37701700 & 3.21248900 & 1.98944000\end{array}$ $\begin{array}{llll}7.37908400 & -2.51930400 & 1.52855900\end{array}$ $\begin{array}{llll}7.11380600 & -3.35945400 & 2.16645500\end{array}$ $\begin{array}{llll}8.45814500 & -2.38722900 & 1.56576300\end{array}$ $\begin{array}{llll}6.90646700 & -1.63309000 & 1.94576600\end{array}$ $\begin{array}{lll}-9.49764500 & 1.15552700 & 0.17492100\end{array}$ $\begin{array}{llll}-9.96639600 & 2.12123100 & 0.35698900\end{array}$ $\begin{array}{lll}-9.83346900 & 0.49336800 & 0.97213700\end{array}$ $\begin{array}{llll}-9.96311200 & 0.60639500 & -1.17556100\end{array}$ $\begin{array}{llll}-11.04524300 & 0.50075200 & -1.20436800\end{array}$ $\begin{array}{llll}-9.65848300 & 1.27205400 & -1.98039400\end{array}$ $\begin{array}{llll}-9.51745300 & -0.36811000 & -1.36436200\end{array}$

Geometry: M052X-D3/def2-TZVPP/toluene (PCM) Frequency: M052X-D3/def2-SVP/toluene (PCM)

Zero-point correction $=$

Thermal correction to Energy=

Thermal correction to Enthalpy=

Thermal correction to Gibbs Free Energy=

Sum of electronic and zero-point Energies=
0.906363

0.962589

0.963533

0.809321

$-2393.584124$

Sum of electronic and thermal Energies=

$-2393.527899$

Sum of electronic and thermal Enthalpies= $-2393.526955$

Sum of electronic and thermal Free Energies=

$-2393.681166$

Single Point: DSD-BLYP-D3/def2-TZVPP/toluene (SMD)

Dispersion correction $\quad-0.091362925$

FINAL SINGLE POINT ENERGY $\quad-2392.097884900254$

\section{G-Red / all-saddle}

$\mathrm{C}$

C

C

C

C

$\mathrm{H}$

$\mathrm{H}$

C

$\mathrm{H}$

$\mathrm{N}$ $\begin{array}{llll}0.26688100 & -1.85378400 & 2.04898400\end{array}$ $\begin{array}{llll}1.23503400 & -1.05367100 & 1.47823400\end{array}$ $\begin{array}{lll}0.62931800 & -0.32261500 & 0.43585800\end{array}$ $\begin{array}{llll}-4.24367400 & -0.58952600 & -0.91755200\end{array}$ $\begin{array}{lll}-5.21472500 & -1.42200600 & -0.32777900\end{array}$ $\begin{array}{llll}-4.60358700 & -2.12610600 & 0.69139500\end{array}$ $\begin{array}{llll}-3.25102200 & -1.71269800 & 0.69964600\end{array}$ $\begin{array}{llll}-2.19897200 & -2.10670000 & 1.50337700\end{array}$ $\begin{array}{llll}-0.93202800 & -1.58713500 & 1.34352000\end{array}$ $\begin{array}{llll}0.42885800 & -2.83328900 & 3.16121400\end{array}$ $\begin{array}{llll}0.37924000 & -3.85462500 & 2.78464600\end{array}$ $\begin{array}{llll}-0.35109500 & -2.71649600 & 3.91116700\end{array}$ $\begin{array}{llll}1.39235100 & -2.69830200 & 3.64357400\end{array}$ $\begin{array}{llll}1.32462800 & 0.59580400 & -0.50659400\end{array}$ $\begin{array}{llll}2.19855200 & 0.98671200 & 0.00384300\end{array}$ $\begin{array}{llll}0.67432700 & 1.42180200 & -0.78185000\end{array}$ $\begin{array}{llll}-4.48321800 & 0.40829300 & -1.99653200\end{array}$ $\begin{array}{llll}-3.62210200 & 0.46817200 & -2.65648300\end{array}$ $\begin{array}{llll}-5.34382300 & 0.07339000 & -2.56588600\end{array}$ $\begin{array}{llll}-5.22268300 & -3.11896900 & 1.61614900\end{array}$ $\begin{array}{llll}-6.27017700 & -3.26056100 & 1.36747300\end{array}$ $\begin{array}{llll}-5.16055600 & -2.78079000 & 2.64966600\end{array}$ $\begin{array}{llll}-4.72266300 & -4.08405000 & 1.55161400\end{array}$ $\begin{array}{lll}-0.66591300 & -0.64007200 & 0.36178800\end{array}$ $\begin{array}{llll}-3.06986200 & -0.76917700 & -0.30348300\end{array}$ $\begin{array}{llll}-1.72111300 & -0.06638100 & -0.62455700\end{array}$ $\begin{array}{llll}-1.83914900 & 1.31376500 & -0.44408200\end{array}$ $\begin{array}{llll}-1.34782200 & -0.33152700 & -1.94289200\end{array}$ $\begin{array}{llll}1.98698400 & -3.78436300 & -1.28272400\end{array}$ $\begin{array}{llll}1.29085500 & -2.66052400 & -1.69115800\end{array}$ $\begin{array}{llll}2.14876500 & -1.55404300 & -1.56447400\end{array}$ $\begin{array}{llll}6.95794100 & -1.70777300 & 0.03228500\end{array}$ $\begin{array}{llll}7.65295900 & -2.88251800 & 0.43218100\end{array}$ $\begin{array}{llll}6.79041800 & -3.94376200 & 0.31355900\end{array}$ $\begin{array}{llll}5.56153900 & -3.41289000 & -0.16311200\end{array}$ $\begin{array}{llll}4.37266800 & -4.04289500 & -0.45238200\end{array}$ $\begin{array}{llll}3.27581400 & -3.33703600 & -0.91794300\end{array}$ $\begin{array}{llll}1.50014800 & -5.19349100 & -1.23466800\end{array}$ $\begin{array}{llll}2.02195400 & -5.81467000 & -1.96157000\end{array}$ $\begin{array}{llll}1.65746600 & -5.63112200 & -0.25011000\end{array}$ $\begin{array}{llll}0.43807600 & -5.23073000 & -1.45860000\end{array}$ $\begin{array}{llll}1.78551500 & -0.12974300 & -1.80399300\end{array}$ $\begin{array}{llll}2.62879800 & 0.41246200 & -2.22312600\end{array}$ $\begin{array}{llll}0.96768100 & -0.11738700 & -2.51659500\end{array}$ $\begin{array}{llll}7.42418600 & -0.34931500 & -0.01483600\end{array}$ $\begin{array}{llll}7.05786700 & -5.37937700 & 0.61449700\end{array}$ $\begin{array}{llll}8.08008900 & -5.50985400 & 0.95673800\end{array}$ $\begin{array}{llll}6.38807700 & -5.74659200 & 1.39049200\end{array}$ $\begin{array}{llll}6.91177700 & -5.99689200 & -0.27020900\end{array}$ $\begin{array}{llll}3.33854400 & -1.96340300 & -1.10542300\end{array}$ $\begin{array}{llll}5.70803900 & -2.04446800 & -0.32259300\end{array}$ $\begin{array}{llll}4.58269600 & -1.08464900 & -0.80885400\end{array}$ $\begin{array}{lll}4.29113700 & -0.15499100 & 0.19562300\end{array}$ 
Geometry: M052X-D3/def2-TZVP/toluene (PCM) Frequency: M052X-D3/def2-SVP/toluene (PCM)

Zero-point correction=

Thermal correction to Energy=

Thermal correction to Enthalpy=

Thermal correction to Gibbs Free Energy=

Sum of electronic and zero-point Energies=
0.927435

0.988602

0.989546

0.821602

$-2913.330418$
Sum of electronic and thermal Energies=

$-2913.269251$ Sum of electronic and thermal Enthalpies= $-2913.268307$ Sum of electronic and thermal Free Energies $=\quad-2913.436250$

\section{Single Point: DSD-BLYP-D3/def2-TZVPP/toluene (SMD)}

Dispersion correction $\quad-0.104229159$

FINAL SINGLE POINT ENERGY - -2914.771455310408

\section{G-Red / staggered-saddle}

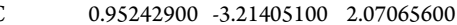

$\begin{array}{llll}\text { C } & 1.68564500 & -2.12158100 & 1.65885600\end{array}$

$\begin{array}{llll}\text { C } & 0.80112400 & -1.22305300 & 1.02609800\end{array}$

$\begin{array}{lllll}\text { C } & -4.19451000 & -1.86101900 & 0.52644900\end{array}$

$\begin{array}{llll}\text { C } & -4.93246900 & -2.97006800 & 0.98067400\end{array}$

$\begin{array}{lll}-4.04429900 & -3.85846900 & 1.55957900\end{array}$

$\begin{array}{lll}-2.76572200 & -3.26574600 & 1.44770300\end{array}$

$\begin{array}{lll}-1.52756400 & -3.70916300 & 1.87386100\end{array}$

$\begin{array}{llll}-0.38808300 & -2.95695800 & 1.68952300\end{array}$

$\begin{array}{llll}1.44511700 & -4.43853600 & 2.76371500\end{array}$

$\begin{array}{llll}1.31955000 & -5.31750900 & 2.13256500\end{array}$

$\begin{array}{llll}0.90210200 & -4.61378300 & 3.69083500\end{array}$

$\begin{array}{llll}2.50034400 & -4.33752800 & 2.99880700\end{array}$

$\begin{array}{llll}1.18469200 & 0.04218300 & 0.34259500\end{array}$

$\begin{array}{llll}2.09589900 & 0.40217900 & 0.80827200\end{array}$

$\begin{array}{llll}0.40508900 & 0.78964100 & 0.46005600\end{array}$

$\begin{array}{llll}-4.72322400 & -0.62499500 & -0.10774600\end{array}$

$\begin{array}{llll}-3.96253400 & -0.16919700 & -0.73571300\end{array}$

$\begin{array}{llll}-5.58297000 & -0.87722600 & -0.72312600\end{array}$

$\begin{array}{llll}-4.34822900 & -5.17376700 & 2.19375800\end{array}$

$\begin{array}{llll}-5.39314600 & -5.42992800 & 2.04669600\end{array}$

$\begin{array}{llll}-4.15430800 & -5.14360200 & 3.26510300\end{array}$

$\begin{array}{llll}-3.73792100 & -5.96791700 & 1.76737300\end{array}$

$\begin{array}{llll}-0.43731800 & -1.72125000 & 1.05303900\end{array}$

$\begin{array}{llll}-2.90039400 & -2.04112200 & 0.80923200\end{array}$

$\begin{array}{lll}-1.72383000 & -1.09128700 & 0.44855400\end{array}$

$\begin{array}{llll}-1.94457100 & 0.17768800 & 0.98502400\end{array}$

$\begin{array}{llll}-1.60235600 & -0.99090900 & -0.93991000\end{array}$

$\begin{array}{llll}2.24240000 & -3.70308700 & -1.91597400\end{array}$

$\begin{array}{llll}1.33308900 & -2.66332100 & -1.82504000\end{array}$

$\begin{array}{llll}2.04211000 & -1.50259500 & -1.47317900\end{array}$

$\begin{array}{llll}7.05446300 & -1.19850200 & -0.76650900\end{array}$

$\begin{array}{llll}7.96715900 & -2.28354300 & -0.88299900\end{array}$

$\begin{array}{llll}7.24797500 & -3.40647900 & -1.20765000\end{array}$

$\begin{array}{llll}5.88761700 & -3.00351700 & -1.29156300\end{array}$

$\begin{array}{llll}4.75968600 & -3.73518000 & -1.58348300\end{array}$

$\begin{array}{llll}3.50593700 & -3.14658600 & -1.62124800\end{array}$

$\begin{array}{llll}1.96919400 & -5.12511800 & -2.27327300\end{array}$

$\begin{array}{llll}2.21479500 & -5.31905900 & -3.31698300\end{array}$

$\begin{array}{llll}2.55473900 & -5.80760300 & -1.66074900\end{array}$

$\begin{array}{llll}0.91669300 & -5.35278200 & -2.12922600\end{array}$

$\begin{array}{llll}1.44839300 & -0.16922300 & -1.17588700\end{array}$

$\begin{array}{llll}2.10091800 & 0.62535400 & -1.52787700\end{array}$

$\begin{array}{llll}0.50231000 & -0.10958100 & -1.70365300\end{array}$

$\begin{array}{llll}7.31648600 & 0.17520600 & -0.43581400\end{array}$

$\begin{array}{llll}7.76064100 & -4.78748100 & -1.43784300\end{array}$

$\begin{array}{llll}8.83713100 & -4.81907500 & -1.29898700\end{array}$

$\begin{array}{llll}7.30538900 & -5.49334600 & -0.74505300\end{array}$

$\begin{array}{llll}7.53621500 & -5.12268600 & -2.44915800\end{array}$

$\begin{array}{llll}3.34498900 & -1.79393200 & -1.35579700\end{array}$

$\begin{array}{llll}5.81487200 & -1.64738400 & -1.01742400\end{array}$

$\begin{array}{llll}4.49468800 & -0.82286000 & -0.97783200\end{array}$

$\begin{array}{lll}4.29889700 & -0.31490800 & 0.31125200\end{array}$ 
Zero-point correction $=$ $\begin{array}{lll}0.71008900 & -3.61167200 & 1.92922600\end{array}$ $\begin{array}{lll}1.71842300 & -2.72573900 & 1.58845700\end{array}$ $\begin{array}{llll}1.11737800 & -1.68044600 & 0.85163500\end{array}$ $\begin{array}{lll}-3.81234900 & -1.26892500 & -0.27010500\end{array}$ $\begin{array}{llll}-4.82263600 & -2.19235500 & 0.07550700\end{array}$ $\begin{array}{lll}-4.21090300 & -3.21523800 & 0.78335400\end{array}$ $\begin{array}{llll}-2.83505400 & -2.89823200 & 0.85193800\end{array}$ $\begin{array}{lll}-1.78336600 & -3.57162500 & 1.44373600\end{array}$ $\begin{array}{lll}-0.49212500 & -3.08694500 & 1.39880500\end{array}$ $\begin{array}{llll}0.86549700 & -4.88389000 & 2.69000700\end{array}$ $\begin{array}{llll}-0.09340400 & -5.35405600 & 2.88741600\end{array}$ $\begin{array}{lll}1.35934800 & -4.70572800 & 3.64381400\end{array}$ $\begin{array}{llll}1.47995600 & -5.58978400 & 2.13128400\end{array}$ $\begin{array}{llll}1.80683700 & -0.52572000 & 0.21422900\end{array}$ $\begin{array}{llll}2.68663500 & -0.27317900 & 0.79866300\end{array}$ $\begin{array}{lll}1.14228800 & 0.33420800 & 0.19974900\end{array}$ $\begin{array}{llll}-3.98986400 & 0.02572900 & -0.98349000\end{array}$ $\begin{array}{llll}-4.83672800 & -0.05660700 & -1.65819800\end{array}$ $\begin{array}{llll}-3.10143700 & 0.24525400 & -1.56972200\end{array}$ $\begin{array}{llll}-4.87963700 & -4.40998500 & 1.37253000\end{array}$ $\begin{array}{llll}-5.59400100 & -4.10893000 & 2.13862700\end{array}$ $\begin{array}{llll}-4.16369600 & -5.08836200 & 1.82721000\end{array}$ $\begin{array}{llll}-5.43080200 & -4.95887200 & 0.61059400\end{array}$ $\begin{array}{llll}-0.20074500 & -1.89721000 & 0.74798400\end{array}$ $\begin{array}{llll}-2.62808800 & -1.69805400 & 0.18957300\end{array}$ $\begin{array}{lll}-1.24897800 & -0.99866600 & 0.03474900\end{array}$ $\begin{array}{llll}-1.28085800 & 0.26346500 & 0.63237800\end{array}$ $\begin{array}{llll}-0.92862200 & -0.86660300 & -1.31771200\end{array}$ $\begin{array}{llll}3.91762900 & -4.10049800 & -1.53901300\end{array}$ $\begin{array}{llll}2.76026600 & -3.33527700 & -1.56950500\end{array}$ $\begin{array}{llll}3.14673200 & -1.99537800 & -1.36213200\end{array}$ $\begin{array}{llll}7.88624500 & -0.32188900 & -0.62684300\end{array}$ $\begin{array}{llll}9.05676700 & -1.13877600 & -0.58807200\end{array}$ $\begin{array}{llll}8.64658600 & -2.43916400 & -0.80027400\end{array}$ $\begin{array}{llll}7.23980200 & -2.41308000 & -0.96781900\end{array}$ $6.34861600-3.44107700-1.19136600$ $\begin{array}{llll}4.99000600 & -3.20839500 & -1.31879100\end{array}$ $\begin{array}{llll}4.00667800 & -5.58082100 & -1.69018900\end{array}$ $\begin{array}{llll}3.55910700 & -5.90205200 & -2.62949500\end{array}$ $\begin{array}{llll}5.03649500 & -5.92545600 & -1.67164800\end{array}$ $\begin{array}{llll}3.46840400 & -6.08089400 & -0.88500700\end{array}$ $\begin{array}{llll}2.25060200 & -0.81237600 & -1.24401700\end{array}$ $\begin{array}{llll}2.76058400 & 0.07194600 & -1.61696400\end{array}$ $\begin{array}{llll}1.36372500 & -0.97867000 & -1.84832400\end{array}$ $\begin{array}{llll}7.72009400 & 1.10226700 & -0.49244100\end{array}$ $\begin{array}{llll}9.52013400 & -3.64586900 & -0.86958500\end{array}$ $\begin{array}{llll}10.17197300 & -3.59924200 & -1.74152400\end{array}$ $\begin{array}{llll}10.15504700 & -3.71683900 & 0.01173500\end{array}$ $\begin{array}{llll}8.93492100 & -4.55795900 & -0.93787500\end{array}$ $\begin{array}{llll}4.47974100 & -1.92453000 & -1.21977300\end{array}$ $\begin{array}{llll}6.81343700 & -1.10378700 & -0.86306000\end{array}$ $\begin{array}{llll}5.33116400 & -0.64725500 & -0.98666800\end{array}$ $\begin{array}{llll}4.92737900 & -0.00908700 & 0.18971300\end{array}$ 
$\begin{array}{llll}5.18251100 & 0.23131800 & -2.06174100\end{array}$ $\begin{array}{llll}-1.97722300 & -4.50202000 & 1.95462900\end{array}$ $\begin{array}{llll}6.71815300 & -4.45180400 & -1.26408500\end{array}$ $\begin{array}{llll}8.64739500 & 1.96502700 & -0.04423600\end{array}$ $\begin{array}{llll}9.61068200 & 1.59503700 & 0.27120400\end{array}$ $\begin{array}{llll}6.74508800 & 1.47108400 & -0.76871700\end{array}$ $\begin{array}{llll}8.48076100 & 3.40035700 & 0.09396000\end{array}$ $\begin{array}{llll}9.53692000 & 4.16100900 & 0.59832700\end{array}$ $\begin{array}{llll}7.31269700 & 4.08755500 & -0.25370700\end{array}$ $\begin{array}{llll}9.44996800 & 5.52835400 & 0.75765700\end{array}$ $\begin{array}{llll}10.45692400 & 3.66163300 & 0.87099600\end{array}$ $\begin{array}{llll}7.20379700 & 5.45033800 & -0.10224900\end{array}$ $\begin{array}{llll}6.46712400 & 3.54958500 & -0.65506300\end{array}$ $\begin{array}{llll}8.26998600 & 6.21410700 & 0.41778400\end{array}$ $\begin{array}{llll}10.30040100 & 6.06221600 & 1.14637800\end{array}$ $\begin{array}{llll}6.28324000 & 5.92899500 & -0.39014000\end{array}$ $\begin{array}{llll}8.15717600 & 7.56471300 & 0.58518900\end{array}$ $\begin{array}{llll}6.99455300 & 8.25465200 & 0.06946900\end{array}$ $\begin{array}{llll}6.08381000 & 7.89217700 & 0.54414100\end{array}$ $\begin{array}{llll}7.08836200 & 9.31162100 & 0.29018200\end{array}$ $\begin{array}{llll}6.89259300 & 8.13187800 & -1.01162700\end{array}$ $\begin{array}{llll}9.32231700 & 8.33307000 & 0.96339700\end{array}$ $\begin{array}{llll}9.04407700 & 9.37764700 & 1.04086400\end{array}$ $\begin{array}{llll}9.70153600 & 8.01324000 & 1.93327200\end{array}$ $\begin{array}{llll}10.12823800 & 8.24011300 & 0.23074800\end{array}$ $\begin{array}{llll}10.47641000 & -0.72294100 & -0.36866300\end{array}$ $\begin{array}{llll}10.66676000 & 0.23378300 & -0.85133700\end{array}$ $\begin{array}{llll}11.12484600 & -1.44608600 & -0.86239000\end{array}$ $\begin{array}{llll}1.37149400 & -3.82590100 & -1.82791000\end{array}$ $\begin{array}{llll}1.27920900 & -4.84476500 & -1.45071900\end{array}$ $\begin{array}{llll}0.65336800 & -3.22283700 & -1.27645500\end{array}$ $\begin{array}{llll}1.01874000 & -3.79290400 & -3.31684000\end{array}$ $\begin{array}{llll}1.71503300 & -4.40122300 & -3.89167500\end{array}$ $\begin{array}{llll}0.01024300 & -4.16519300 & -3.48485000\end{array}$ $\begin{array}{llll}1.07367700 & -2.77403400 & -3.69564400\end{array}$ $\begin{array}{llll}3.16074700 & -2.82166200 & 1.97060000\end{array}$ $\begin{array}{llll}3.43173100 & -3.87307400 & 2.06860900\end{array}$ $\begin{array}{llll}3.78432600 & -2.41109000 & 1.17966800\end{array}$ $\begin{array}{llll}3.45728700 & -2.08711400 & 3.28012800\end{array}$ $\begin{array}{llll}2.85647100 & -2.49067800 & 4.09352500\end{array}$ $\begin{array}{llll}4.50859000 & -2.17766000 & 3.54504700\end{array}$ $\begin{array}{llll}3.22138700 & -1.02910000 & 3.18242300\end{array}$ $\begin{array}{llll}10.84864200 & -0.65979000 & 1.11595600\end{array}$ $\begin{array}{llll}10.74626500 & -1.64364900 & 1.56981300\end{array}$ $\begin{array}{lll}11.87656700 & -0.32688400 & 1.24629400\end{array}$ $\begin{array}{llll}10.19317900 & 0.01770500 & 1.65930500\end{array}$ $\begin{array}{llll}10.26737500 & -2.10443000 & -0.30026700\end{array}$ $\begin{array}{llll}-6.59273100 & -1.06655900 & -0.29183500\end{array}$ $\begin{array}{lll}-6.86065300 & -2.62465400 & 0.45198700\end{array}$ $\begin{array}{llll}-6.54111900 & -2.70700200 & -1.68015000\end{array}$ $\begin{array}{llll}-7.59832200 & -2.64050800 & -1.92854200\end{array}$ $\begin{array}{llll}-5.97803100 & -2.17455700 & -2.44433900\end{array}$ $\begin{array}{llll}-6.24092600 & -3.75313600 & -1.71060900\end{array}$ $\begin{array}{llll}-4.23723400 & 1.22174200 & -0.02811200\end{array}$ $\begin{array}{llll}-5.42074100 & 1.02972500 & 0.85408600\end{array}$ $\begin{array}{llll}-3.35461000 & 1.35867800 & 0.58950700\end{array}$ $\begin{array}{llll}-4.37469900 & 2.11198200 & -0.63599300\end{array}$ $\begin{array}{llll}-5.43419200 & 0.43474200 & 2.13499700\end{array}$ $\begin{array}{llll}-6.66768800 & 1.36366500 & 0.49401200\end{array}$ $\begin{array}{llll}-6.75499100 & 0.40222900 & 2.55163700\end{array}$ $\begin{array}{lll}-4.23920400 & -0.01905100 & 2.91072400\end{array}$ $\begin{array}{llll}-7.52449400 & 0.98547700 & 1.51774300\end{array}$ $\begin{array}{llll}-7.12162800 & 2.02122400 & -0.84069300\end{array}$ $\begin{array}{llll}-7.26581800 & -0.15462800 & 3.83643000\end{array}$ $\begin{array}{llll}-3.48812500 & -0.42423900 & 2.23627900\end{array}$ $\begin{array}{lll}-4.53548200 & -0.83182600 & 3.57407900\end{array}$ $\begin{array}{llll}-3.62116700 & 1.11635300 & 3.72996400\end{array}$ $\begin{array}{lll}-8.88584500 & 1.19855800 & 1.42170600\end{array}$ $\begin{array}{llll}-8.65958700 & 2.20824800 & -0.74771900\end{array}$ $\begin{array}{llll}-6.81057900 & 1.18222300 & -1.91641200\end{array}$

$\begin{array}{llll}-6.49903800 & 3.25458700 & -1.01745600\end{array}$

$\begin{array}{llll}-7.04456700 & -1.21957700 & 3.90478100\end{array}$ $\begin{array}{lll}-8.33995300 & -0.02666200 & 3.93274300\end{array}$ $\begin{array}{llll}-6.78862800 & 0.33231700 & 4.68548200\end{array}$ $\begin{array}{llll}-2.76067900 & 0.76269300 & 4.29398500\end{array}$ $\begin{array}{llll}-3.29131200 & 1.92021200 & 3.07452400\end{array}$ $\begin{array}{lll}-4.34881500 & 1.52960900 & 4.42647100\end{array}$ $\begin{array}{llll}-9.45056000 & 1.79989900 & 0.31425600\end{array}$ $\begin{array}{llll}-9.52368600 & 0.89074200 & 2.23552600\end{array}$ $\begin{array}{llll}-9.45284000 & 2.76550300 & -1.66843300\end{array}$ $\begin{array}{lll}-10.79445800 & 2.12540800 & 0.01990700\end{array}$ $\begin{array}{llll}-10.79291100 & 2.73621300 & -1.22521200\end{array}$ $\begin{array}{llll}-8.92393900 & 3.32745100 & -2.93870900\end{array}$ $\begin{array}{llll}-11.98083800 & 1.87349700 & 0.88677700\end{array}$ $\begin{array}{llll}-11.98421900 & 3.22507300 & -1.98507100\end{array}$ $\begin{array}{llll}-9.73795100 & 3.55257500 & -3.62008200\end{array}$ $\begin{array}{llll}-8.24252700 & 2.62372500 & -3.41051100\end{array}$ $\begin{array}{lll}-11.71893400 & 1.29016400 & 1.76469900\end{array}$ $\begin{array}{llll}-12.75040600 & 1.33262700 & 0.33834900\end{array}$ $\begin{array}{lll}-12.41803200 & 2.81295300 & 1.22369400\end{array}$ $\begin{array}{llll}-12.76633500 & 3.50214900 & -1.27897400\end{array}$ $\begin{array}{llll}-11.72840100 & 4.13086300 & -2.53358600\end{array}$ $\begin{array}{llll}-12.52169100 & 2.17013100 & -2.95506500\end{array}$ $\begin{array}{llll}-12.80742100 & 1.26904000 & -2.41533000\end{array}$ $\begin{array}{llll}-13.39123900 & 2.54030500 & -3.49449100\end{array}$ $\begin{array}{llll}-11.75833200 & 1.89339000 & -3.68005300\end{array}$ $\begin{array}{llll}-8.36505400 & 4.24130800 & -2.74177800\end{array}$

Geometry: M052X-D3/def2-TZVP/toluene (PCM) Frequency: M052X-D3/def2-SVP/toluene (PCM)

Zero-point correction $=$

Thermal correction to Energy=

Thermal correction to Enthalpy= Thermal correction to Gibbs Free Energy= Sum of electronic and zero-point Energies= Sum of electronic and thermal Energies= Sum of electronic and thermal Enthalpies= Sum of electronic and thermal Free Energies=

Single Point: DSD-BLYP-D3/def2-TZVPP/toluene (SMD)

Dispersion correction $-0.135493612$

FINAL SINGLE POINT ENERGY $\quad-3386.157089925156$
1.270487

1.349456

1.350400

1.145144

$-3384.341248$

$-3384.262279$

$-3384.261335$

$-3384.466591$

\section{G-Red $\left.\right|^{\mathrm{Et}} /$ staggered-saddle}

C $\quad 1.02551600 \quad-4.25533200 \quad 2.06553800$ $\begin{array}{llll}1.90141100 & -3.24493800 & 1.70062600\end{array}$ $\begin{array}{llll}1.12399400 & -2.20812800 & 1.14065200\end{array}$ $\begin{array}{llll}-3.91105100 & -2.22807000 & 0.57014600\end{array}$ $\begin{array}{llll}-4.79241400 & -3.25603200 & 0.96796200\end{array}$ $\begin{array}{llll}-4.01607600 & -4.25942600 & 1.52560900\end{array}$ $\begin{array}{llll}-2.67309200 & -3.81778300 & 1.46444800\end{array}$ $\begin{array}{llll}-1.50312300 & -4.42595900 & 1.87354500\end{array}$ $\begin{array}{llll}-0.27364800 & -3.81346900 & 1.72444800\end{array}$ $\begin{array}{llll}1.38725800 & -5.56326100 & 2.68192300\end{array}$ $\begin{array}{llll}0.50660600 & -6.14881400 & 2.92898100\end{array}$ $\begin{array}{llll}1.96298000 & -5.41428000 & 3.59396400\end{array}$ $\begin{array}{llll}2.00266100 & -6.14952800 & 1.99963900\end{array}$ $\begin{array}{llll}1.62085100 & -0.93252300 & 0.55580500\end{array}$ $\begin{array}{llll}2.53591900 & -0.64484300 & 1.06477000\end{array}$ $\begin{array}{llll}0.88156300 & -0.14950200 & 0.70585800\end{array}$ $\begin{array}{llll}-4.28351900 & -0.92958900 & -0.05314700\end{array}$ 

$\begin{array}{llll}-4.50337600 & -5.55006300 & 2.09019500\end{array}$ $\begin{array}{llll}-5.13295000 & -5.37320000 & 2.96160300\end{array}$ $\begin{array}{lll}-3.68165500 & -6.19227900 & 2.39330200\end{array}$ $\begin{array}{lll}-5.10280300 & -6.08857700 & 1.35793100\end{array}$ $\begin{array}{llll}-0.17186100 & -2.55076700 & 1.16062900\end{array}$ $\begin{array}{llll}-2.65091800 & -2.56332800 & 0.87288700\end{array}$ $\begin{array}{llll}-1.37379500 & -1.70650900 & 0.66212800\end{array}$ $\begin{array}{llll}-1.46606800 & -0.52120500 & 1.39792200\end{array}$ $\begin{array}{llll}-1.21756900 & -1.39526900 & -0.69215000\end{array}$ $\begin{array}{llll}3.81935400 & -4.08559300 & -1.80031000\end{array}$ $\begin{array}{llll}2.60332000 & -3.44266700 & -1.61692900\end{array}$ $\begin{array}{llll}2.89125100 & -2.09802700 & -1.30600100\end{array}$ $\begin{array}{llll}7.51705500 & -0.03744600 & -0.90888100\end{array}$ $\begin{array}{llll}8.75147700 & -0.72928600 & -1.09773900\end{array}$ $\begin{array}{llll}8.43586600 & -2.03517800 & -1.41378000\end{array}$ $\begin{array}{llll}7.02266800 & -2.13543100 & -1.41930100\end{array}$ $\begin{array}{llll}6.20576500 & -3.22079300 & -1.65635600\end{array}$ $\begin{array}{llll}4.82684500 & -3.11580400 & -1.60231500\end{array}$ $\begin{array}{llll}4.02165500 & -5.52639400 & -2.12450800\end{array}$ $\begin{array}{llll}3.50387400 & -5.79349500 & -3.04452800\end{array}$ $\begin{array}{llll}5.07380800 & -5.76654800 & -2.24697900\end{array}$ $3.62136700-6.15661500-1.33042600$ $\begin{array}{llll}1.91295800 & -1.02949800 & -0.96366900\end{array}$ $\begin{array}{llll}2.29387900 & -0.06627300 & -1.29290700\end{array}$ $\begin{array}{llll}0.97938200 & -1.22668800 & -1.48209300\end{array}$ $\begin{array}{llll}7.24178900 & 1.33835100 & -0.58399800\end{array}$ $\begin{array}{llll}9.39739400 & -3.13230800 & -1.72307100\end{array}$ $\begin{array}{llll}9.90598800 & -2.94311900 & -2.66811000\end{array}$ $\begin{array}{llll}10.15918200 & -3.21100200 & -0.94981100\end{array}$ $\begin{array}{llll}8.89465100 & -4.09175200 & -1.79969300\end{array}$ $\begin{array}{llll}4.22001500 & -1.90674500 & -1.30404500\end{array}$ $\begin{array}{llll}6.49735600 & -0.89498600 & -1.11586000\end{array}$ $\begin{array}{llll}4.97693200 & -0.58137200 & -1.01929500\end{array}$ $\begin{array}{llll}4.66617700 & -0.11500200 & 0.26140900\end{array}$ $\begin{array}{lllll}4.62014300 & 0.38718800 & -1.96047600\end{array}$ $\begin{array}{llll}-1.54946700 & -5.40622700 & 2.32188500\end{array}$ $\begin{array}{llll}6.65216700 & -4.17569100 & -1.88530500\end{array}$ $\begin{array}{llll}8.13520700 & 2.23510100 & -0.13390000\end{array}$ $\begin{array}{llll}9.15794600 & 1.93188500 & 0.02881200\end{array}$ $\begin{array}{llll}6.21117100 & 1.63284100 & -0.70275100\end{array}$ $\begin{array}{llll}7.85789000 & 3.61990800 & 0.20227400\end{array}$ $\begin{array}{llll}8.88708000 & 4.41530800 & 0.70868500\end{array}$ $\begin{array}{llll}6.60543400 & 4.22403500 & 0.04945400\end{array}$ $\begin{array}{llll}8.69470400 & 5.73642500 & 1.05549000\end{array}$ $\begin{array}{llll}9.87028000 & 3.98158900 & 0.83235700\end{array}$ $\begin{array}{llll}6.39116400 & 5.53970400 & 0.38915900\end{array}$ $\begin{array}{llll}5.77654500 & 3.65797800 & -0.34809000\end{array}$ $\begin{array}{llll}7.43059300 & 6.33613200 & 0.91407300\end{array}$ $\begin{array}{llll}9.52854400 & 6.30108100 & 1.43650000\end{array}$ $\begin{array}{llll}5.40831900 & 5.95542500 & 0.24558300\end{array}$ $\begin{array}{llll}7.21465600 & 7.63678300 & 1.27124200\end{array}$ $\begin{array}{llll}5.95201100 & 8.26577200 & 0.94971000\end{array}$ $\begin{array}{llll}5.12584300 & 7.75440700 & 1.44160700\end{array}$ $\begin{array}{llll}5.97045200 & 9.28931600 & 1.30567000\end{array}$ $\begin{array}{llll}5.76121700 & 8.27400700 & -0.12642900\end{array}$ $\begin{array}{llll}8.33906800 & 8.46676800 & 1.64290600\end{array}$ $\begin{array}{llll}7.97744100 & 9.45886900 & 1.88715700\end{array}$ $\begin{array}{llll}8.84363600 & 8.06712200 & 2.52168400\end{array}$ $\begin{array}{llll}9.06995600 & 8.55090000 & 0.83437200\end{array}$ $\begin{array}{llll}10.14450200 & -0.19508800 & -0.99372200\end{array}$ $\begin{array}{llll}10.18486300 & 0.82389500 & -1.37394400\end{array}$ $\begin{array}{llll}10.78705800 & -0.78746000 & -1.64446300\end{array}$ $\begin{array}{llll}1.24267500 & -4.04307400 & -1.77303500\end{array}$ $\begin{array}{llll}1.28728000 & -5.09819800 & -1.50201100\end{array}$ $\begin{array}{llll}0.54524700 & -3.57284400 & -1.08319100\end{array}$ $\begin{array}{llll}0.71262400 & -3.89968100 & -3.20164000\end{array}$ $\begin{array}{llll}1.38645400 & -4.37533000 & -3.91230600\end{array}$ $\begin{array}{llll}-0.27156000 & -4.35363800 & -3.29758500\end{array}$
Geometry: M052X-D3/def2-TZVP/toluene (PCM) Frequency: M052X-D3/def2-SVP/toluene (PCM)

Zero-point correction= 
Thermal correction to Gibbs Free Energy= Sum of electronic and zero-point Energies= Sum of electronic and thermal Energies= Sum of electronic and thermal Enthalpies=

1.143854 $-3384.338871$ $-3384.259713$ $-3384.258769$ Sum of electronic and thermal Free Energies $=\quad-3384.465329$

Single Point: DSD-BLYP-D3/def2-TZVPP/toluene (SMD)

Dispersion correction $\quad-0.132736501$

FINAL SINGLE POINT ENERGY $\quad-3386.154352816919$

3G-Red / all-saddle

$\begin{array}{llll}-3.85377400 & -2.40318000 & -2.08388500\end{array}$

C $\quad-4.65611800-1.44284400-1.50283100$

$\begin{array}{llll}\text { C } & -3.88819700 & -0.77295500 & -0.52889500\end{array}$

$\begin{array}{lllll}\text { C } & 0.95635100 & -1.73761600 & 0.59457800\end{array}$

$\begin{array}{lllll}\text { C } & 1.75631700 & -2.72577200 & -0.00858800\end{array}$

C $\quad 0.98955200 \quad-3.36319000 \quad-0.96574500$

$\begin{array}{llll}\text { C } & -0.28266400 & -2.74866700 & -0.92314200\end{array}$

C $\quad-1.42539000 \quad-3.00465100 \quad-1.65699900$

$\begin{array}{lllll}\text { C } & -2.58990600 & -2.29569900 & -1.45363400\end{array}$

$\begin{array}{lllll}\text { C } & -4.22727000 & -3.39383100 & -3.13386800\end{array}$

$\mathrm{H} \quad-4.38019500 \quad-4.37886600 \quad-2.69339500$

$\mathrm{H} \quad-3.45138800 \quad-3.48437700 \quad-3.89128100$

$\mathrm{H} \quad-5.15102200 \quad-3.09644000 \quad-3.62116300$

$\begin{array}{lllll}\text { C } & -4.38061700 & 0.28438800 & 0.39570400\end{array}$

$\begin{array}{llll}-5.21260200 & 0.78165100 & -0.09158100\end{array}$

$\begin{array}{lll}-3.59746600 & 1.01153300 & 0.59375500\end{array}$

$\begin{array}{lll}1.40731700 & -0.75194300 & 1.61589400\end{array}$

$\begin{array}{lll}0.61021600 & -0.54834900 & 2.32586300\end{array}$

$\begin{array}{llll}2.24805200 & -1.19115800 & 2.14245800\end{array}$

$1.39314600-4.48754500-1.85875600$

$\begin{array}{llll}2.47614900 & -4.55559100 & -1.91040700\end{array}$

$\begin{array}{llll}1.00873900 & -4.35010600 & -2.86718200\end{array}$

$\begin{array}{llll}1.01399100 & -5.43751700 & -1.48334200\end{array}$

$\begin{array}{llll}-2.65430000 & -1.28339800 & -0.50321800\end{array}$

$\begin{array}{llll}-0.26436600 & -1.75615900 & 0.04860100\end{array}$

$\begin{array}{lll}-1.46687600 & -0.83335600 & 0.39353600\end{array}$

$\begin{array}{lll}-1.14773200 & 0.49971200 & 0.12188100\end{array}$

$\begin{array}{lll}-1.79916100 & -0.96489200 & 1.74210900\end{array}$

$\begin{array}{lll}-5.76477200 & -3.87392800 & 1.43389100\end{array}$

$\begin{array}{lll}-4.85284500 & -2.88103000 & 1.74981500\end{array}$

$\begin{array}{llll}-5.49910500 & -1.64203400 & 1.60385800\end{array}$

$\begin{array}{lll}-10.34141900 & -0.97123300 & 0.26251600\end{array}$

$\begin{array}{llll}-11.26225600 & -2.01261000 & -0.03858500\end{array}$

$\begin{array}{llll}-10.60307200 & -3.20952600 & 0.09326500\end{array}$

$\begin{array}{llll}-9.27158700 & -2.89596000 & 0.47834900\end{array}$

$\begin{array}{llll}-8.20168600 & -3.72205800 & 0.73573600\end{array}$

$\begin{array}{llll}-6.96726200 & -3.21198100 & 1.10534900\end{array}$

$\begin{array}{llll}-5.55020500 & -5.35009100 & 1.45209000\end{array}$

$\begin{array}{llll}-5.98797600 & -5.79703200 & 2.34405400\end{array}$

$\begin{array}{llll}-6.00202800 & -5.82664800 & 0.58450700\end{array}$

$\begin{array}{llll}-4.48734200 & -5.57497200 & 1.45139800\end{array}$

$\begin{array}{lll}-4.87062900 & -0.29965900 & 1.75086300\end{array}$

$\begin{array}{lll}-5.57263800 & 0.40125000 & 2.19500000\end{array}$

$\begin{array}{llll}-4.01517900 & -0.40987700 & 2.40886500\end{array}$

$\begin{array}{llll}-10.54550700 & 0.45071500 & 0.25872800\end{array}$

$\begin{array}{llll}-11.14759900 & -4.58236000 & -0.11260300\end{array}$

$\begin{array}{llll}-12.18446500 & -4.53378600 & -0.43163000\end{array}$

$\begin{array}{llll}-10.58045900 & -5.11748300 & -0.87242900\end{array}$

$\begin{array}{llll}-11.09960900 & -5.16196200 & 0.80789800\end{array}$

$\begin{array}{llll}-6.76751700 & -1.84374300 & 1.22189100\end{array}$

$\begin{array}{llll}-9.15634400 & -1.51846400 & 0.57326500\end{array}$ $\begin{array}{lll}-7.84420800 & -0.76396300 & 0.93682000\end{array}$

$\begin{array}{llll}-7.45393300 & 0.04111800 & -0.13967000\end{array}$ $\begin{array}{llll}-8.03963200 & 0.03652800 & 2.06098200\end{array}$

$\begin{array}{llll}-1.40651500 & -3.78252000 & -2.40622200\end{array}$ $\begin{array}{llll}-8.32847900 & -4.79133400 & 0.65113200\end{array}$ $\begin{array}{llll}-12.29162600 & -1.88053500 & -0.31777400\end{array}$ $\begin{array}{llll}-5.68974600 & -1.23081000 & -1.71432200\end{array}$ $\begin{array}{llll}-3.82328900 & -3.00350900 & 2.03809500\end{array}$ $\begin{array}{llll}2.78662000 & -2.91275800 & 0.23983700\end{array}$ $\begin{array}{llll}-11.71904800 & 1.01978100 & -0.06447200\end{array}$ $\begin{array}{llll}-12.54727700 & 0.37709500 & -0.33698700\end{array}$ $\begin{array}{llll}-9.69425800 & 1.05362000 & 0.53215900\end{array}$ $\begin{array}{llll}-12.01215600 & 2.43808600 & -0.09290400\end{array}$ $\begin{array}{lll}-13.28686100 & 2.86672000 & -0.46855700\end{array}$ $\begin{array}{llll}-11.08222000 & 3.42796400 & 0.24398800\end{array}$ $\begin{array}{llll}-13.63015400 & 4.20130700 & -0.51574600\end{array}$ $\begin{array}{llll}-14.03144800 & 2.12698600 & -0.72943500\end{array}$ $\begin{array}{llll}-11.40267600 & 4.76440300 & 0.20363900\end{array}$ $\begin{array}{llll}-10.08492400 & 3.15019700 & 0.54992200\end{array}$ $\begin{array}{llll}-12.68967700 & 5.19386600 & -0.18506700\end{array}$ $\begin{array}{llll}-14.63022600 & 4.47174000 & -0.80902200\end{array}$ $\begin{array}{llll}-10.65029000 & 5.48391100 & 0.47842200\end{array}$ $\begin{array}{llll}-13.00600100 & 6.51914100 & -0.23666600\end{array}$ $\begin{array}{llll}-12.05677400 & 7.50338400 & 0.23634200\end{array}$ $\begin{array}{llll}-11.13323300 & 7.46417900 & -0.34033700\end{array}$ $\begin{array}{llll}-12.48631400 & 8.49087900 & 0.11482000\end{array}$ $\begin{array}{llll}-11.81145200 & 7.35791800 & 1.29101400\end{array}$ $\begin{array}{llll}-14.37047700 & 6.91774900 & -0.50222600\end{array}$ $\begin{array}{llll}-14.42924700 & 7.99983200 & -0.49049400\end{array}$ $\begin{array}{llll}-14.69515000 & 6.57264700 & -1.48362200\end{array}$ $\begin{array}{llll}-15.06162600 & 6.52413600 & 0.24701300\end{array}$ $\begin{array}{llll}1.86589800 & 0.59359700 & 0.98436100\end{array}$ $\begin{array}{llll}2.49106200 & 0.40489100 & -0.35343100\end{array}$ $\begin{array}{llll}0.99847400 & 1.23209000 & 0.85432300\end{array}$ $\begin{array}{llll}2.56378400 & 1.07499000 & 1.66432600\end{array}$ $\begin{array}{llll}1.82604000 & 0.44539600 & -1.59546700\end{array}$ $\begin{array}{llll}3.77398600 & 0.08591100 & -0.54419400\end{array}$ $\begin{array}{llll}2.74447800 & 0.12636800 & -2.57513300\end{array}$ $\begin{array}{llll}0.78088800 & 0.66837900 & -1.72010300\end{array}$ $\begin{array}{llll}3.97324200 & -0.09505600 & -1.90731400\end{array}$ $\begin{array}{llll}4.88014500 & -0.04922200 & 0.53813200\end{array}$ $\begin{array}{llll}2.50924100 & 0.00007500 & -4.04208800\end{array}$ $\begin{array}{llll}5.21518500 & -0.43186300 & -2.40423300\end{array}$ $\begin{array}{llll}6.18718100 & -0.45307700 & -0.19808600\end{array}$ $\begin{array}{llll}4.53100000 & -1.03073100 & 1.46717600\end{array}$ $\begin{array}{llll}5.05380900 & 1.17135800 & 1.19504600\end{array}$ $\begin{array}{llll}1.53865300 & 0.41048100 & -4.30403600\end{array}$ $\begin{array}{llll}2.52715300 & -1.04654100 & -4.34538200\end{array}$ $\begin{array}{llll}3.27134500 & 0.52573900 & -4.61405800\end{array}$ $6.30510900-0.60781100-1.57377400$ $\begin{array}{llll}5.33802100 & -0.56420400 & -3.46936500\end{array}$ $\begin{array}{llll}7.38253900 & -0.69745900 & 0.34828400\end{array}$ $\begin{array}{llll}7.64059400 & -0.96389600 & -1.87448600\end{array}$ $\begin{array}{llll}8.30438400 & -1.02447100 & -0.66424500\end{array}$ $\begin{array}{llll}7.68969100 & -0.55358300 & 1.79858100\end{array}$ $\begin{array}{llll}8.20426900 & -1.19104100 & -3.23631900\end{array}$ $\begin{array}{llll}9.34107300 & -1.25633300 & -0.49246800\end{array}$ $\begin{array}{llll}8.03034700 & 0.91247700 & 2.19362400\end{array}$ $\begin{array}{llll}8.54939300 & -1.17943000 & 2.01348200\end{array}$ $\begin{array}{llll}6.84916700 & -0.88761100 & 2.40070000\end{array}$ $\begin{array}{llll}8.35518600 & -0.24337500 & -3.75274900\end{array}$ $\begin{array}{llll}7.54168100 & -1.80127500 & -3.84662800\end{array}$ $\begin{array}{llll}9.16532400 & -1.69199200 & -3.16661500\end{array}$ $\begin{array}{llll}8.71109100 & 1.64929700 & 1.09403500\end{array}$ $\begin{array}{llll}8.65514000 & 0.89670100 & 3.08238200\end{array}$ $\begin{array}{llll}7.10796200 & 1.43803100 & 2.41774000\end{array}$ $\begin{array}{llll}8.07811700 & 2.43372000 & 0.10669800\end{array}$ $\begin{array}{llll}10.02127600 & 1.59265000 & 0.84868400\end{array}$ $\begin{array}{llll}9.04684300 & 2.85815100 & -0.77767200\end{array}$ $\begin{array}{llll}7.02038700 & 2.62769600 & 0.06892500\end{array}$ 


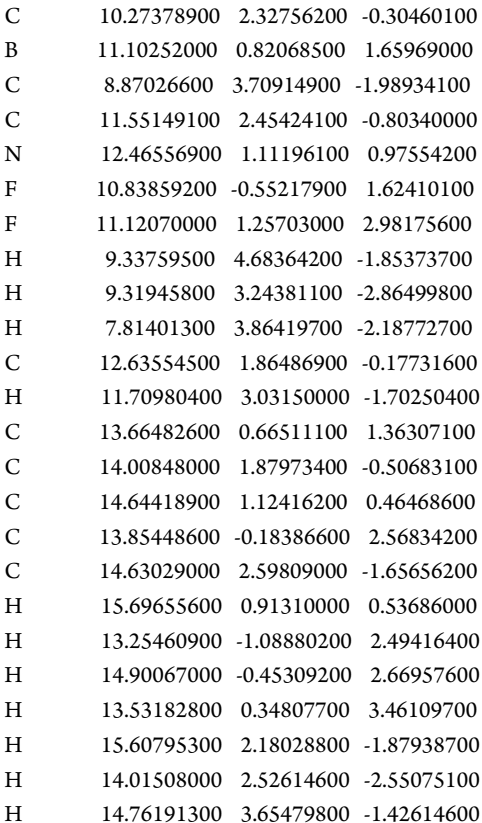

Geometry: M052X-D3/def2-TZVP/toluene (PCM) Frequency: M052X-D3/def2-SVP/toluene (PCM)

Zero-point correction=

1.176704

Thermal correction to Energy=

1.254743

Thermal correction to Enthalpy=

1.255687

Thermal correction to Gibbs Free Energy=

Sum of electronic and zero-point Energies=

Sum of electronic and thermal Energies=

1.048672

$-3749.850893$

$-3749.772853$

$-3749.771909$

Sum of electronic and thermal Free Energies $=\quad-3749.978924$

Single Point: DSD-BLYP-D3/def2-TZVPP/toluene (SMD)

Dispersion correction $\quad-0.135256551$

FINAL SINGLE POINT ENERGY $\quad-3751.697457156848$
$1.63331600-5.01286500-1.10014800$ $\begin{array}{llll}2.70417400 & -5.02459000 & -1.28173100\end{array}$ $\begin{array}{llll}1.12730600 & -5.31544700 & -2.01406700\end{array}$ $\begin{array}{llll}1.41466400 & -5.75676100 & -0.33461400\end{array}$ $\begin{array}{lll}1.566661600 & -1.74161800 & -0.64203600\end{array}$ $\begin{array}{llll}-0.11839100 & -1.86473400 & -0.18116000\end{array}$ $\begin{array}{lll}-1.37670700 & -0.97051300 & -0.00564400\end{array}$ $\begin{array}{llll}-1.19226800 & 0.25803300 & -0.64213200\end{array}$ $\begin{array}{llll}-1.61932000 & -0.75369400 & 1.35354500\end{array}$ $\begin{array}{llll}-5.41125900 & -3.54123500 & 2.35932700\end{array}$ $\begin{array}{lll}-4.53659300 & -2.48027400 & 2.20143200\end{array}$ $\begin{array}{llll}-5.26232000 & -1.40141900 & 1.66805300\end{array}$ $\begin{array}{llll}-10.22383000 & -1.39413200 & 0.63292800\end{array}$ $\begin{array}{llll}-11.10600400 & -2.49131800 & 0.83235300\end{array}$ $\begin{array}{llll}-10.37175400 & -3.53667000 & 1.33610400\end{array}$ $\begin{array}{llll}-9.03331800 & -3.07315300 & 1.44516600\end{array}$ $\begin{array}{llll}-7.90068300 & -3.71828900 & 1.88767500\end{array}$ $\begin{array}{llll}-6.67203300 & -3.07953500 & 1.92187800\end{array}$ $\begin{array}{llll}-5.11010900 & -4.90148400 & 2.89292900\end{array}$ $\begin{array}{llll}-5.57079500 & -5.05154600 & 3.86868000\end{array}$ $\begin{array}{llll}-5.48214500 & -5.67771800 & 2.22646900\end{array}$ $\begin{array}{llll}-4.03740600 & -5.03183300 & 3.00298000\end{array}$ $\begin{array}{llll}-4.70459200 & -0.08253500 & 1.25855500\end{array}$ $\begin{array}{lll}-5.41595300 & 0.71113400 & 1.47108600\end{array}$ $\begin{array}{llll}-3.80303400 & 0.08541100 & 1.83818700\end{array}$ $\begin{array}{llll}-10.50651200 & -0.08317900 & 0.11694600\end{array}$ $\begin{array}{llll}-10.85403200 & -4.89715900 & 1.71080900\end{array}$ $\begin{array}{llll}-11.91446700 & -4.99425000 & 1.49862600\end{array}$ $\begin{array}{llll}-10.32185600 & -5.66747600 & 1.15511000\end{array}$ $\begin{array}{llll}-10.69898300 & -5.08621400 & 2.77186000\end{array}$ $\begin{array}{llll}-6.54185000 & -1.76224200 & 1.50462300\end{array}$ $\begin{array}{llll}-8.98814600 & -1.75921300 & 1.00859500\end{array}$ $\begin{array}{llll}-7.69703500 & -0.89297000 & 0.94079600\end{array}$ $\begin{array}{llll}-7.43729600 & -0.54194400 & -0.38883200\end{array}$ $\begin{array}{llll}-7.84838200 & 0.26654900 & 1.69991100\end{array}$ $\begin{array}{llll}-1.26473100 & -4.71477900 & -1.58999400\end{array}$ $\begin{array}{llll}-7.97252000 & -4.74360200 & 2.21953600\end{array}$ $\begin{array}{llll}-12.16070300 & -2.49645400 & 0.62511100\end{array}$ $\begin{array}{llll}-5.65887400 & -2.26915300 & -1.56055900\end{array}$ $\begin{array}{llll}-3.48295000 & -2.45877900 & 2.41995900\end{array}$ $\begin{array}{llll}3.03409100 & -2.68466200 & 0.09116900\end{array}$ $\begin{array}{llll}-11.73314300 & 0.29724900 & -0.27714800\end{array}$ $\begin{array}{llll}-12.54448900 & -0.41636300 & -0.20149000\end{array}$ $\begin{array}{llll}-9.67005100 & 0.59474400 & 0.05618400\end{array}$ $\begin{array}{llll}-12.10631100 & 1.59100700 & -0.81209000\end{array}$ $\begin{array}{llll}-13.43029800 & 1.81917800 & -1.19163200\end{array}$ $\begin{array}{llll}-11.20661900 & 2.65055800 & -0.97366900\end{array}$ $\begin{array}{llll}-13.84862800 & 3.02602900 & -1.71119300\end{array}$ $\begin{array}{llll}-14.15310800 & 1.02354800 & -1.07273700\end{array}$ $\begin{array}{llll}-11.60201300 & 3.86207400 & -1.49015500\end{array}$ $\begin{array}{llll}-10.17327200 & 2.52959000 & -0.68583700\end{array}$ $\begin{array}{llll}-12.93832400 & 4.08440700 & -1.88505900\end{array}$ $\begin{array}{llll}-14.88358400 & 3.14557600 & -1.98198400\end{array}$ $\begin{array}{llll}-10.86993100 & 4.64563400 & -1.58699200\end{array}$ $\begin{array}{llll}-13.32940000 & 5.27901600 & -2.41567500\end{array}$ $\begin{array}{llll}-12.40253600 & 6.38984500 & -2.44125600\end{array}$ $\begin{array}{llll}-11.51875300 & 6.14594800 & -3.02927500\end{array}$ $\begin{array}{llll}-12.88835500 & 7.24024400 & -2.90519700\end{array}$ $\begin{array}{llll}-12.08092600 & 6.67717200 & -1.43711100\end{array}$ $\begin{array}{llll}-14.73412600 & 5.52530500 & -2.65719900\end{array}$ $\begin{array}{llll}-14.85036600 & 6.52139200 & -3.06813000\end{array}$ $\begin{array}{llll}-15.13655200 & 4.81440800 & -3.37818400\end{array}$ $\begin{array}{llll}-15.32282700 & 5.45610900 & -1.73893800\end{array}$ $\begin{array}{llll}1.97610500 & 0.70566400 & -0.38881100\end{array}$ $\begin{array}{llll}2.23612000 & 2.06097800 & 0.16295900\end{array}$ $\begin{array}{llll}2.86940200 & 0.30686500 & -0.86085300\end{array}$ $\begin{array}{llll}1.18285200 & 0.77292400 & -1.12901500\end{array}$ $\begin{array}{llll}1.28122300 & 3.06708500 & 0.41245800\end{array}$ $\begin{array}{llll}3.43736900 & 2.47307900 & 0.57441400\end{array}$ $\begin{array}{lll}1.93584800 & 4.13081200 & 1.00162300\end{array}$ 

$\begin{array}{llll}4.77441700 & 1.68370500 & 0.49907100\end{array}$ $\begin{array}{lll}1.35468000 & 5.42896000 & 1.44990600\end{array}$ $\begin{array}{llll}4.37788100 & 4.42313300 & 1.63216000\end{array}$ $\begin{array}{lll}5.86573600 & 2.59687200 & 1.12527300\end{array}$ $\begin{array}{llll}5.09867100 & 1.39414200 & -0.82828900\end{array}$ $\begin{array}{llll}4.66477100 & 0.49053500 & 1.21616700\end{array}$ $\begin{array}{llll}0.28504000 & 5.44600400 & 1.26470700\end{array}$ $\begin{array}{llll}1.80699800 & 6.26469600 & 0.91821100\end{array}$ $\begin{array}{llll}1.51915500 & 5.58411200 & 2.51501200\end{array}$ $\begin{array}{llll}5.63989900 & 3.86257000 & 1.64990300\end{array}$ $\begin{array}{llll}4.23433200 & 5.41195500 & 2.04304100\end{array}$ $\begin{array}{llll}7.16540600 & 2.31900100 & 1.25913400\end{array}$ $\begin{array}{llll}6.86785800 & 4.37609600 & 2.13020200\end{array}$ $\begin{array}{llll}7.81651600 & 3.40266100 & 1.88004600\end{array}$ $\begin{array}{llll}7.78962800 & 1.07517300 & 0.73694900\end{array}$ $\begin{array}{lll}7.06800900 & 5.70474800 & 2.77742900\end{array}$ $\begin{array}{llll}8.86813100 & 3.44219500 & 2.10540500\end{array}$ $\begin{array}{llll}8.36058900 & 1.32479200 & -0.67792800\end{array}$ $\begin{array}{llll}7.05776100 & 0.27326500 & 0.69853500\end{array}$ $\begin{array}{llll}8.60441100 & 0.77934400 & 1.39241300\end{array}$ $\begin{array}{llll}6.51191200 & 5.77227200 & 3.71127000\end{array}$ $\begin{array}{lll}6.72992500 & 6.51163700 & 2.12947800\end{array}$ $\begin{array}{llll}8.11976500 & 5.86216200 & 2.99621100\end{array}$ $8.97848800 \quad 0.08390600 \quad-1.21343300$ $\begin{array}{llll}9.09626600 & 2.12351400 & -0.63699500\end{array}$ $\begin{array}{llll}7.54595700 & 1.62773600 & -1.33082400\end{array}$ $\begin{array}{llll}8.32644300 & -0.97028600 & -1.88650900\end{array}$ $\begin{array}{llll}10.26971200 & -0.21577900 & -1.05993400\end{array}$ $\begin{array}{llll}9.26758900 & -1.94432700 & -2.15290000\end{array}$ $\begin{array}{llll}7.27987800 & -0.98567200 & -2.13728900\end{array}$ $\begin{array}{llll}10.49294000 & -1.46348400 & -1.62836700\end{array}$ $\begin{array}{llll}11.36620000 & 0.67052100 & -0.39911700\end{array}$ $\begin{array}{llll}9.06651700 & -3.24897000 & -2.84666500\end{array}$ $\begin{array}{llll}11.74535000 & -2.03998500 & -1.60715800\end{array}$ $\begin{array}{llll}12.67918300 & -0.15954700 & -0.42456000\end{array}$ $\begin{array}{llll}11.01177900 & 0.97481600 & 0.91626300\end{array}$ $\begin{array}{llll}11.53057000 & 1.85207000 & -1.12066600\end{array}$ $\begin{array}{llll}9.72080700 & -3.33672800 & -3.71248300\end{array}$ $\begin{array}{llll}9.28250800 & -4.08272300 & -2.18013500\end{array}$ $\begin{array}{llll}8.03901100 & -3.34142100 & -3.18490300\end{array}$ $12.82280400-1.40679700-1.01667900$ $\begin{array}{llll}11.88523100 & -3.01269500 & -2.05590200\end{array}$ $\begin{array}{llll}13.86269600 & 0.20155600 & 0.07940800\end{array}$ $\begin{array}{llll}14.16414200 & -1.82429400 & -0.86622900\end{array}$ $\begin{array}{llll}14.80630000 & -0.81037800 & -0.17709600\end{array}$ $\begin{array}{llll}14.07090600 & 1.48492200 & 0.79952700\end{array}$ $\begin{array}{llll}14.74980900 & -3.09859500 & -1.37354100\end{array}$ $\begin{array}{llll}15.83967600 & -0.78227000 & 0.12112600\end{array}$ $\begin{array}{llll}13.50237600 & 1.49180300 & 1.72779700\end{array}$ $\begin{array}{llll}15.12403200 & 1.61611200 & 1.02517600\end{array}$ $\begin{array}{llll}13.72235300 & 2.32046900 & 0.19666000\end{array}$ $\begin{array}{llll}15.71904600 & -3.27681700 & -0.91700100\end{array}$ $14.10531800-3.94655000-1.15104600$ $\begin{array}{llll}14.88772500 & -3.05889900 & -2.45349500\end{array}$

Geometry: M052X-D3/def2-TZVP/toluene (PCM) Frequency: B3LYP-D3/6-31g(d,p)/toluene (PCM)

Zero-point correction $=$

1.160532

Thermal correction to Energy=

Thermal correction to Enthalpy=

Thermal correction to Gibbs Free Energy=

Sum of electronic and zero-point Energies=

Sum of electronic and thermal Energies=

Sum of electronic and thermal Enthalpies=

Sum of electronic and thermal Free Energies=
Single Point: DSD-BLYP-D3/def2-TZVPP/toluene (SMD)

\begin{tabular}{ll} 
Dispersion correction & -0.130144992 \\
\hline & FINAL SINGLE POINT ENERGY
\end{tabular}

\section{G-Red| ${ }^{\mathrm{Et}} /$ all-saddle}

$\begin{array}{llll}C & -5.07871000 & -4.34920700 & -0.90972800\end{array}$

$\begin{array}{lllll}\mathrm{C} & -5.71589000 & -3.12647500 & -0.77835100\end{array}$

$\begin{array}{lllll}\text { C } & -4.72652900 & -2.18155600 & -0.42723300\end{array}$

$\begin{array}{lllll}\text { C } & 0.21993600 & -3.12165200 & 0.17456800\end{array}$

$\begin{array}{llll}\text { C } & 0.84797100 & -4.38004600 & 0.05609000\end{array}$

$\begin{array}{lllll}\text { C } & -0.13974400 & -5.29630900 & -0.27017200\end{array}$

C $\quad-1.35565500 \quad-4.57830500 \quad-0.33901700$

$\begin{array}{lllll}\text { C } & -2.63927000 & -5.00134600 & -0.62834200\end{array}$

$\begin{array}{llll}\text { C } & -3.70753500 & -4.12686500 & -0.64176100\end{array}$

C $\quad-5.72032100 \quad-5.65265300 \quad-1.24372400$

$\mathrm{H} \quad-4.98335000 \quad-6.43060900-1.42051000$

$\mathrm{H} \quad-6.33810600 \quad-5.56043600-2.13524300$

$\begin{array}{lllll}\mathrm{H} & -6.36792400 & -5.97904300 & -0.42978000\end{array}$

C $\quad-4.93759600 \quad-0.74313700 \quad-0.10825800$

$\begin{array}{llll}-5.78566200 & -0.37368100 & -0.67728400\end{array}$

$\begin{array}{llll}-4.05687400 & -0.17235300 & -0.39047600\end{array}$

$\begin{array}{llll}0.87998400 & -1.81529100 & 0.44655700\end{array}$

$\begin{array}{llll}1.76627200 & -1.98535000 & 1.05081100\end{array}$

$\begin{array}{llll}0.20285700 & -1.16963300 & 0.99988100\end{array}$

$\begin{array}{llll}0.04969900 & -6.75377700 & -0.52028300\end{array}$

$\begin{array}{llll}0.65473000 & -6.91295400 & -1.41303400\end{array}$

$\begin{array}{llll}-0.89836300 & -7.26450200 & -0.66024900\end{array}$

$\begin{array}{llll}0.56867600 & -7.22386300 & 0.31334300\end{array}$

$\begin{array}{llll}-3.53046300 & -2.78138400 & -0.35459400\end{array}$

$\begin{array}{llll}-1.09458400 & -3.24641400 & -0.05587500\end{array}$

$\begin{array}{lll}-2.16782000 & -2.12510900 & 0.00221000\end{array}$

$\begin{array}{llll}-1.86627500 & -1.11905900 & -0.91830000\end{array}$

$\begin{array}{lll}-2.21666100 & -1.58220200 & 1.28792200\end{array}$

$\begin{array}{llll}-7.72047300 & -2.84318300 & 2.75557300\end{array}$

$\begin{array}{lll}-6.39703800 & -2.53074500 & 2.47745600\end{array}$

$\begin{array}{llll}-6.38818100 & -1.23806300 & 1.91551400\end{array}$

$\begin{array}{lll}-10.44831800 & 1.69189000 & 1.07192700\end{array}$

$\begin{array}{lll}-11.80066900 & 1.34088800 & 1.36368300\end{array}$

$\begin{array}{lll}-11.77710200 & 0.07669300 & 1.91762000\end{array}$

$\begin{array}{llll}-10.42220000 & -0.33536300 & 1.96431300\end{array}$

$\begin{array}{lll}-9.86028000 & -1.51287200 & 2.41090400\end{array}$

$\begin{array}{llll}-8.49364000 & -1.72899100 & 2.36128900\end{array}$

$\begin{array}{lll}-8.22616900 & -4.11715500 & 3.34222600\end{array}$

$\begin{array}{llll}-7.74863300 & -4.31818900 & 4.29998400\end{array}$

$\begin{array}{llll}-9.30037000 & -4.08901200 & 3.49994400\end{array}$

$\begin{array}{llll}-8.00309300 & -4.95706500 & 2.68422000\end{array}$

$\begin{array}{llll}-5.20753400 & -0.49202800 & 1.39777800\end{array}$

$\begin{array}{llll}-5.36034400 & 0.57506500 & 1.53645100\end{array}$

$\begin{array}{llll}-4.32670400 & -0.78867300 & 1.95958900\end{array}$

$\begin{array}{llll}-9.87890100 & 2.88936400 & 0.51124700\end{array}$

$\begin{array}{llll}-12.95345200 & -0.70059300 & 2.40408500\end{array}$

$\begin{array}{llll}-13.38822500 & -0.22974200 & 3.28540300\end{array}$

$\begin{array}{lll}-13.72846700 & -0.74834800 & 1.64126200\end{array}$

$\begin{array}{lll}-12.67793500 & -1.71709600 & 2.66894000\end{array}$

$\begin{array}{llll}-7.64180600 & -0.76203700 & 1.85334600\end{array}$

$\begin{array}{llll}-9.64155900 & 0.67954700 & 1.44775000\end{array}$

$\begin{array}{llll}-8.09285200 & 0.62393600 & 1.31901200\end{array}$

$\begin{array}{lll}-7.71972300 & 0.76790900 & -0.02043300\end{array}$

$\begin{array}{llll}-7.50625400 & 1.64330700 & 2.07252600\end{array}$

$\begin{array}{llll}-2.81193800 & -6.04329100 & -0.84943900\end{array}$

$\begin{array}{lll}-10.50131500 & -2.28549400 & 2.80510300\end{array}$

$\begin{array}{llll}-10.55397800 & 3.86749500 & -0.11510000\end{array}$ 

$\begin{array}{llll}-9.97279100 & 5.07146500 & -0.68088000\end{array}$ $\begin{array}{llll}-10.79202100 & 5.96449600 & -1.37306600\end{array}$ $\begin{array}{llll}-8.61805500 & 5.40418200 & -0.57484100\end{array}$ $\begin{array}{llll}-10.30323800 & 7.12192000 & -1.94332800\end{array}$ $\begin{array}{llll}-11.84653500 & 5.74232900 & -1.46616300\end{array}$ $\begin{array}{llll}-8.10959900 & 6.55319500 & -1.13447600\end{array}$ $\begin{array}{llll}-7.94195200 & 4.75589000 & -0.03817600\end{array}$ $\begin{array}{llll}-8.93807300 & 7.44563300 & -1.84635000\end{array}$ $\begin{array}{llll}-10.98496400 & 7.77442400 & -2.46174300\end{array}$ $\begin{array}{llll}-7.05985700 & 6.76223100 & -1.01818100\end{array}$ $\begin{array}{llll}-8.43068900 & 8.57738800 & -2.41646600\end{array}$ $\begin{array}{llll}-7.06079300 & 8.96023100 & -2.15108100\end{array}$ $\begin{array}{llll}-6.36493600 & 8.20746000 & -2.51994800\end{array}$ $\begin{array}{llll}-6.85142100 & 9.89064600 & -2.66576200\end{array}$ $\begin{array}{llll}-6.87804700 & 9.10490400 & -1.08357200\end{array}$ $\begin{array}{llll}-9.32907200 & 9.53620900 & -3.01947200\end{array}$ $\begin{array}{llll}-8.74723900 & 10.35580900 & -3.42488800\end{array}$ $\begin{array}{llll}-9.88832900 & 9.08377200 & -3.83783600\end{array}$ $\begin{array}{llll}-10.04250500 & 9.93957400 & -2.29609500\end{array}$ $\begin{array}{lll}-13.04073000 & 2.14755600 & 1.14266800\end{array}$ $\begin{array}{lll}-12.83900600 & 3.20234400 & 1.31968300\end{array}$ $\begin{array}{llll}-13.77901400 & 1.84986600 & 1.88698300\end{array}$ $\begin{array}{llll}-5.20035600 & -3.38030600 & 2.76586200\end{array}$ $\begin{array}{llll}-5.49189400 & -4.42982700 & 2.71515900\end{array}$ $\begin{array}{lll}-4.44184900 & -3.23023800 & 2.00053700\end{array}$ $\begin{array}{llll}-4.59774700 & -3.07861200 & 4.13985000\end{array}$ $\begin{array}{llll}-5.33381600 & -3.23555200 & 4.92660000\end{array}$ $\begin{array}{llll}-3.73818000 & -3.71659200 & 4.33514000\end{array}$ $\begin{array}{llll}-4.27049900 & -2.04183100 & 4.19047400\end{array}$ $\begin{array}{llll}-7.16545700 & -2.84300100 & -1.01322000\end{array}$ $\begin{array}{llll}-7.74589500 & -3.73002900 & -0.75828700\end{array}$ $\begin{array}{llll}-7.50193100 & -2.04933400 & -0.34966400\end{array}$ $\begin{array}{llll}-7.44321200 & -2.44138300 & -2.46347800\end{array}$ $\begin{array}{llll}-7.12695700 & -3.22640500 & -3.14851500\end{array}$ $\begin{array}{llll}-8.50341900 & -2.25150100 & -2.61669500\end{array}$ $\begin{array}{llll}-6.89544200 & -1.53554100 & -2.71670200\end{array}$ $\begin{array}{llll}-13.63665400 & 1.94115000 & -0.25307000\end{array}$ $\begin{array}{llll}-13.91761800 & 0.89868900 & -0.39002200\end{array}$ $\begin{array}{llll}-14.52329300 & 2.55661600 & -0.39323100\end{array}$ $\begin{array}{llll}-12.91694500 & 2.18776700 & -1.03096900\end{array}$ $\begin{array}{llll}2.29582600 & -4.66814100 & 0.29666700\end{array}$ $\begin{array}{llll}2.90284400 & -3.82888000 & -0.03704900\end{array}$ $\begin{array}{llll}2.59207200 & -5.52776800 & -0.30485300\end{array}$ $\begin{array}{llll}2.58659600 & -4.94604300 & 1.77323200\end{array}$ $\begin{array}{llll}3.64262900 & -5.15729800 & 1.92750800\end{array}$ $\begin{array}{llll}2.31955000 & -4.08295200 & 2.38025900\end{array}$ $\begin{array}{llll}2.00575900 & -5.79645900 & 2.12665900\end{array}$ $\begin{array}{llll}1.30172900 & -1.07071200 & -0.84637800\end{array}$ $\begin{array}{llll}2.22817800 & -1.86441700 & -1.69962000\end{array}$ $\begin{array}{llll}0.40731400 & -0.83645400 & -1.41581900\end{array}$ $\begin{array}{llll}1.78000100 & -0.13911400 & -0.55549700\end{array}$ $\begin{array}{llll}1.87403200 & -2.75626900 & -2.73516000\end{array}$ $\begin{array}{llll}3.55852200 & -1.86178000 & -1.53707200\end{array}$ $\begin{array}{llll}3.05035700 & -3.31908100 & -3.20441100\end{array}$ $\begin{array}{llll}0.49433900 & -3.00107400 & -3.25773800\end{array}$ $\begin{array}{llll}4.10325700 & -2.75373300 & -2.44838200\end{array}$ $\begin{array}{llll}4.37916400 & -1.02156200 & -0.51941600\end{array}$ $\begin{array}{llll}3.17600700 & -4.33690100 & -4.28629900\end{array}$ $\begin{array}{llll}-0.23017400 & -2.91531500 & -2.45078700\end{array}$ $\begin{array}{llll}0.43080400 & -4.02496300 & -3.62705500\end{array}$ $\begin{array}{llll}0.12355400 & -2.02437500 & -4.37592400\end{array}$ $\begin{array}{llll}5.46698900 & -2.96464800 & -2.50292800\end{array}$ $\begin{array}{llll}5.87469900 & -1.38711600 & -0.73019600\end{array}$ $\begin{array}{llll}3.99081100 & -1.33324800 & 0.78581600\end{array}$ $\begin{array}{llll}4.17740200 & 0.34054000 & -0.75335200\end{array}$ $\begin{array}{llll}2.66677100 & -5.25818900 & -4.00393900\end{array}$ $\begin{array}{llll}4.21432800 & -4.57617300 & -4.49661800\end{array}$ $\begin{array}{llll}2.71706800 & -3.98019900 & -5.20698100\end{array}$ $\begin{array}{llll}-0.87879800 & -2.22412200 & -4.74874400\end{array}$ $\begin{array}{llll}0.15276500 & -1.00036900 & -4.00835100\end{array}$ $\begin{array}{llll}0.82520700 & -2.10241700 & -5.20470400\end{array}$ $\begin{array}{llll}6.34044400 & -2.30078700 & -1.66368600\end{array}$ $\begin{array}{llll}5.86162400 & -3.66751600 & -3.22011100\end{array}$ $\begin{array}{llll}6.93140100 & -0.89697100 & -0.06704900\end{array}$ $\begin{array}{llll}7.74845900 & -2.37158400 & -1.55539700\end{array}$ $\begin{array}{llll}8.11722700 & -1.49190500 & -0.55005600\end{array}$ $\begin{array}{llll}6.81947600 & 0.15832200 & 0.97730000\end{array}$ $\begin{array}{lllll}8.66419200 & -3.21604900 & -2.37419900\end{array}$ $\begin{array}{llll}9.49949400 & -1.25796200 & -0.02906300\end{array}$ $\begin{array}{llll}7.64106400 & 0.05137100 & 1.67950600\end{array}$ $\begin{array}{llll}5.88428600 & 0.03922000 & 1.51822600\end{array}$ $\begin{array}{lll}6.85472900 & 1.59501800 & 0.39582100\end{array}$ $\begin{array}{llll}8.11621300 & -3.87446700 & -3.04189100\end{array}$ $\begin{array}{lllll}9.29661400 & -3.83033700 & -1.73534400\end{array}$ $\begin{array}{llll}9.32083800 & -2.59136500 & -2.97958200\end{array}$ $\begin{array}{llll}10.21531900 & -1.43285600 & -0.83248900\end{array}$ $\begin{array}{llll}9.61485600 & -0.21866500 & 0.27066100\end{array}$ $\begin{array}{llll}9.82469500 & -2.16583600 & 1.15924700\end{array}$ $\begin{array}{llll}8.08838700 & 1.87229600 & -0.39004500\end{array}$ $\begin{array}{llll}6.78307700 & 2.29451000 & 1.22494000\end{array}$ $\begin{array}{llll}5.98834500 & 1.72941600 & -0.24512500\end{array}$ $\begin{array}{llll}9.73369400 & -3.21410900 & 0.87952300\end{array}$ $\begin{array}{llll}10.83639100 & -1.98787100 & 1.51777700\end{array}$ $\begin{array}{llll}9.13427000 & -1.97736200 & 1.97924500\end{array}$ $\begin{array}{llll}8.27334000 & 1.67404900 & -1.77549400\end{array}$ $\begin{array}{llll}9.22921700 & 2.30402600 & 0.16462200\end{array}$ $\begin{array}{llll}9.59066200 & 1.99911700 & -2.05773700\end{array}$ $\begin{array}{llll}7.22477100 & 1.24708900 & -2.75288800\end{array}$ $\begin{array}{llll}10.18525800 & 2.39276800 & -0.83655500\end{array}$ $\begin{array}{llll}9.47078400 & 2.67685000 & 1.65424400\end{array}$ $\begin{array}{llll}10.25670000 & 1.92707800 & -3.38960000\end{array}$ $\begin{array}{llll}7.70466800 & 0.73032300 & -3.58423600\end{array}$ $\begin{array}{llll}6.55018000 & 0.53120500 & -2.28720800\end{array}$ $\begin{array}{llll}6.41482800 & 2.43343300 & -3.28046500\end{array}$ $\begin{array}{llll}11.47531500 & 2.79929300 & -0.55395100\end{array}$ $\begin{array}{llll}10.96650200 & 3.07425200 & 1.77913400\end{array}$ $\begin{array}{llll}9.20555400 & 1.58225800 & 2.48135900\end{array}$ $\begin{array}{llll}8.65318600 & 3.74534300 & 2.02072600\end{array}$ $\begin{array}{llll}9.70515800 & 2.50588500 & -4.12859900\end{array}$ $\begin{array}{llll}11.27314500 & 2.30752000 & -3.35241800\end{array}$ $\begin{array}{llll}10.29350100 & 0.89677300 & -3.74344500\end{array}$ $\begin{array}{llll}7.06496100 & 3.15815900 & -3.76775100\end{array}$ $\begin{array}{llll}5.66395400 & 2.10256300 & -3.99494200\end{array}$ $\begin{array}{llll}5.90620300 & 2.93832300 & -2.46136200\end{array}$ $\begin{array}{lll}11.86770300 & 3.13159700 & 0.72759600\end{array}$ $\begin{array}{llll}12.19603400 & 2.85070900 & -1.35515100\end{array}$ $\begin{array}{llll}11.60906200 & 3.42106300 & 2.89814100\end{array}$ $\begin{array}{llll}13.12221200 & 3.53939200 & 1.23747700\end{array}$ $\begin{array}{lll}12.95706400 & 3.72232300 & 2.60188100\end{array}$ $\begin{array}{llll}10.94452500 & 3.44147000 & 4.22747500\end{array}$ $\begin{array}{llll}14.37749000 & 3.73234000 & 0.45592600\end{array}$ $\begin{array}{llll}14.00712600 & 4.10602300 & 3.59470900\end{array}$ $\begin{array}{llll}9.93737500 & 3.84076100 & 4.14757600\end{array}$ $\begin{array}{llll}10.86805500 & 2.42900300 & 4.62341700\end{array}$ $\begin{array}{llll}11.51932900 & 4.04250400 & 4.92575800\end{array}$ $\begin{array}{llll}14.73100900 & 4.75880100 & 0.54346800\end{array}$ $\begin{array}{llll}15.16772300 & 3.08089200 & 0.82665800\end{array}$ $\begin{array}{llll}14.22951900 & 3.51701000 & -0.59811700\end{array}$ $\begin{array}{lll}13.60500500 & 4.83595900 & 4.29730600\end{array}$ $\begin{array}{llll}14.82622100 & 4.59799700 & 3.07173200\end{array}$ $\begin{array}{llll}14.54237400 & 2.89690000 & 4.36461600\end{array}$ $\begin{array}{lll}14.97735400 & 2.17233900 & 3.67853300\end{array}$ $\begin{array}{lll}15.30413600 & 3.19550600 & 5.08204400\end{array}$ $\begin{array}{lll}13.73724500 & 2.40159300 & 4.90439700\end{array}$ 
Geometry: M052X-D3/def2-TZVP/toluene (PCM) Frequency: M052X-D3/def2-SVP/toluene (PCM)

Zero-point correction=

1.633723

Thermal correction to Energy=

1.735743

1.736687

1.478636

Thermal correction to Gibbs Free Energy=

Sum of electronic and zero-point Energies=

Sum of electronic and thermal Energies=

Sum of electronic and thermal Enthalpies=

$-4377.868211$

$-4377.766192$

$-4377.765247$

Sum of electronic and thermal Free Energies $=\quad-4378.023298$

Single Point: DSD-BLYP-D3/def2-TZVPP/toluene (SMD)

Dispersion correction $\quad-0.177445326$

FINAL SINGLE POINT ENERGY $\quad-4380.214112497155$
3G-Red $\left.\right|^{\text {Et }} /$ staggered-saddle

C $\quad-3.76529000-4.30922100-1.51614000$

C $\quad-4.72626500 \quad-3.33085400 \quad-1.31495200$

$\begin{array}{lllll}\text { C } & -4.07099600 & -2.22214200 & -0.73685000\end{array}$

$\begin{array}{llll}\text { C } & 0.86544200 & -1.90782000 & 0.35797200\end{array}$

$\begin{array}{llll}1.84155400 & -2.89285000 & 0.09974400\end{array}$

$1.18500300-3.97287500-0.46941200$

$\begin{array}{llll}-0.18162500 & -3.61842600 & -0.55739600\end{array}$

$\begin{array}{llll}-1.26726400 & -4.31965600 & -1.04520300\end{array}$

$\begin{array}{llll}-2.53739600 & -3.77652900 & -1.05913900\end{array}$

$\begin{array}{lll}-3.98923600 & -5.66807000 & -2.08616000\end{array}$

$\begin{array}{llll}-3.05377500 & -6.19355100 & -2.25426300\end{array}$

$\begin{array}{lll}-4.51875000 & -5.60491400 & -3.03510500\end{array}$

$\begin{array}{llll}-4.59860100 & -6.26981000 & -1.41168200\end{array}$

$\begin{array}{lll}-4.69323000 & -0.94246600 & -0.29859900\end{array}$

$\begin{array}{llll}-5.55232600 & -0.73403500 & -0.92969400\end{array}$

$\begin{array}{lll}-3.97767400 & -0.13214400 & -0.41323100\end{array}$

$\begin{array}{llll}1.09774800 & -0.56008900 & 0.94421300\end{array}$

$\begin{array}{lll}1.92062600 & -0.61500100 & 1.65301500\end{array}$

$\begin{array}{llll}0.20875400 & -0.22781500 & 1.47341900\end{array}$

$\begin{array}{llll}1.80335500 & -5.25318200 & -0.91825500\end{array}$

$\begin{array}{llll}2.47928100 & -5.07936000 & -1.75516800\end{array}$

$\begin{array}{llll}1.05352700 & -5.97202100 & -1.23543500\end{array}$

$2.38547900 \quad-5.70187600-0.11510400$

$\begin{array}{llll}-2.76671500 & -2.49257100 & -0.58797000\end{array}$

$\begin{array}{llll}-0.33669600 & -2.34024100 & -0.04161700\end{array}$

$\begin{array}{lll}-1.67551000 & -1.55667900 & -0.00560600\end{array}$

$\begin{array}{lll}-1.57385100 & -0.39905800 & -0.78303500\end{array}$

$\begin{array}{llll}-1.99346100 & -1.20118900 & 1.30891100\end{array}$

$\begin{array}{llll}-7.02795100 & -4.05137300 & 1.98777900\end{array}$

$\begin{array}{lll}-5.82654800 & -3.36094600 & 1.90729700\end{array}$

$\begin{array}{llll}-6.12997900 & -2.05536700 & 1.47232900\end{array}$

$\begin{array}{llll}-10.75536800 & -0.26066100 & 0.38021200\end{array}$

$\begin{array}{llll}-11.97331500 & -1.00002600 & 0.46940900\end{array}$

$\begin{array}{llll}-11.64412400 & -2.26499700 & 0.91288100\end{array}$

$\begin{array}{llll}-10.23935500 & -2.29349700 & 1.09319700\end{array}$

$\begin{array}{llll}-9.41244900 & -3.31866900 & 1.50112300\end{array}$

$\begin{array}{llll}-8.04262400 & -3.14814700 & 1.60377200\end{array}$

$\begin{array}{llll}-7.20680400 & -5.47731300 & 2.38466700\end{array}$

$\begin{array}{llll}-6.79164400 & -5.65914100 & 3.37492400\end{array}$

$\begin{array}{llll}-8.25495300 & -5.76186800 & 2.40000700\end{array}$

$\begin{array}{llll}-6.68793300 & -6.13555700 & 1.68788000\end{array}$

$\begin{array}{llll}-5.16115800 & -0.96319200 & 1.17917700\end{array}$

$\begin{array}{lll}-5.61651100 & -0.00113300 & 1.39930900\end{array}$

$\begin{array}{llll}-4.28904100 & -1.08430400 & 1.81552900\end{array}$

$\begin{array}{llll}-10.49997200 & 1.10295600 & -0.00501900\end{array}$ $\begin{array}{lll}-12.59079400 & -3.38641700 & 1.17912200\end{array}$

$\begin{array}{llll}-13.24639300 & -3.14520900 & 2.01544200\end{array}$

$\begin{array}{lll}-13.22097000 & -3.57654500 & 0.31201800\end{array}$

$\begin{array}{llll}-12.06386800 & -4.30442100 & 1.42095800\end{array}$

$\begin{array}{llll}-7.45541400 & -1.93151400 & 1.29713300\end{array}$

$\begin{array}{llll}-9.73338000 & -1.05077800 & 0.76659000\end{array}$

$\begin{array}{llll}-8.22745200 & -0.66640400 & 0.83536900\end{array}$

$\begin{array}{llll}-7.77944700 & -0.26274000 & -0.42606000\end{array}$

$\begin{array}{llll}-8.03325900 & 0.37401500 & 1.74689600\end{array}$

$\begin{array}{llll}-1.11886700 & -5.31687500 & -1.42963000\end{array}$ $\begin{array}{llll}-9.84251000 & -4.27803600 & 1.74171100\end{array}$

$\begin{array}{llll}-11.37411600 & 1.92781500 & -0.60586300\end{array}$

$\begin{array}{llll}-12.36098100 & 1.56831800 & -0.85269000\end{array}$ $\begin{array}{llll}-9.50170300 & 1.45200500 & 0.20612400\end{array}$

$\begin{array}{llll}-11.11954100 & 3.30093800 & -1.00118900\end{array}$

$\begin{array}{llll}-12.13381000 & 4.02447600 & -1.63079500\end{array}$

$\begin{array}{llll}-9.90535700 & 3.96291400 & -0.78913200\end{array}$

$\begin{array}{llll}-11.96417600 & 5.33200100 & -2.03579800\end{array}$

$\begin{array}{llll}-13.08736700 & 3.54456100 & -1.80502700\end{array}$

$\begin{array}{llll}-9.71362900 & 5.26613200 & -1.18619500\end{array}$

$\begin{array}{llll}-9.08853800 & 3.45239000 & -0.30127200\end{array}$

$\begin{array}{llll}-10.73853400 & 5.99052900 & -1.83090700\end{array}$

$\begin{array}{llll}-12.78592200 & 5.84009400 & -2.51123900\end{array}$

$\begin{array}{llll}-8.76013600 & 5.72841400 & -0.99489500\end{array}$

$\begin{array}{llll}-10.54531900 & 7.27823900 & -2.24009400\end{array}$

$\begin{array}{llll}-9.32938800 & 7.97155900 & -1.87399200\end{array}$

$\begin{array}{llll}-8.45474700 & 7.47394800 & -2.29138100\end{array}$

$\begin{array}{llll}-9.36556100 & 8.97748300 & -2.27570400\end{array}$

$\begin{array}{llll}-9.20611200 & 8.03411700 & -0.78990100\end{array}$

$\begin{array}{llll}-11.66187700 & 8.03455900 & -2.76164500\end{array}$

$\begin{array}{llll}-11.31973400 & 9.02803500 & -3.02675400\end{array}$

$\begin{array}{llll}-12.06484300 & 7.56697600 & -3.65951000\end{array}$

$\begin{array}{llll}-12.46873400 & 8.12766000 & -2.03007600\end{array}$

$\begin{array}{llll}-13.36504000 & -0.54880800 & 0.15783700\end{array}$

$\begin{array}{llll}-13.50256300 & 0.48681000 & 0.46291000\end{array}$

$\begin{array}{llll}-14.05701100 & -1.13647800 & 0.76035900\end{array}$

$\begin{array}{llll}-4.47074700 & -3.88044700 & 2.26659700\end{array}$

$\begin{array}{llll}-4.43699300 & -4.95186400 & 2.06716100\end{array}$

$\begin{array}{llll}-3.71526000 & -3.42072900 & 1.63298600\end{array}$

$\begin{array}{llll}-4.12554000 & -3.61726000 & 3.73404100\end{array}$

$\begin{array}{llll}-4.85968400 & -4.07916400 & 4.39202200\end{array}$

$\begin{array}{llll}-3.14164900 & -4.01252200 & 3.97803400\end{array}$

$\begin{array}{llll}-4.12305500 & -2.54765500 & 3.93541300\end{array}$

$\begin{array}{llll}-6.17215200 & -3.40555000 & -1.68953700\end{array}$

$\begin{array}{llll}-6.50958100 & -4.43827900 & -1.59859900\end{array}$

$\begin{array}{llll}-6.76762500 & -2.82026800 & -0.99236100\end{array}$

$\begin{array}{llll}-6.42357400 & -2.90185600 & -3.11262000\end{array}$

$\begin{array}{llll}-5.84845300 & -3.47923000 & -3.83459000\end{array}$

$\begin{array}{llll}-7.47842000 & -2.97573100 & -3.36866600\end{array}$

$\begin{array}{llll}-6.12357200 & -1.85950900 & -3.20320100\end{array}$

$\begin{array}{llll}-13.72359200 & -0.72764600 & -1.32046500\end{array}$

$\begin{array}{llll}-13.67605300 & -1.78049700 & -1.59226000\end{array}$

$\begin{array}{llll}-14.72941400 & -0.36543000 & -1.52431800\end{array}$

$\begin{array}{llll}-13.02619900 & -0.19481900 & -1.96352300\end{array}$

$\begin{array}{llll}3.29821400 & -2.77689300 & 0.41485700\end{array}$

$\begin{array}{llll}3.62560400 & -1.74896600 & 0.26961000\end{array}$

$\begin{array}{llll}3.86111800 & -3.39028600 & -0.28849800\end{array}$

$\begin{array}{llll}3.62892100 & -3.20588300 & 1.84591000\end{array}$

$\begin{array}{llll}4.69414100 & -3.10064000 & 2.04242500\end{array}$

$\begin{array}{llll}3.09009500 & -2.58953800 & 2.56275700\end{array}$

$\begin{array}{llll}3.34365500 & -4.24307100 & 2.01353100\end{array}$

$\begin{array}{llll}1.46491700 & 0.46710900 & -0.15036900\end{array}$

$\begin{array}{llll}1.66480500 & 1.81693100 & 0.44293200\end{array}$

$\begin{array}{llll}2.36568100 & 0.14762600 & -0.66772100\end{array}$

$\begin{array}{llll}0.65020700 & 0.50976900 & -0.86876400\end{array}$

$\begin{array}{llll}0.65888300 & 2.76040400 & 0.74490300\end{array}$

$\begin{array}{llll}2.86116600 & 2.28917300 & 0.81176600\end{array}$

$\begin{array}{llll}1.29165900 & 3.85624400 & 1.30912800\end{array}$

$\begin{array}{llll}-0.80177600 & 2.60422400 & 0.46283500\end{array}$

$\begin{array}{lll}2.67358800 & 3.55428100 & 1.34968600\end{array}$ 
$\begin{array}{llll}4.22873400 & 1.56608800 & 0.70780100\end{array}$ $\begin{array}{llll}0.64122200 & 5.10722600 & 1.79366500\end{array}$ $\begin{array}{lll}-1.36478600 & 3.20918800 & 1.17309600\end{array}$ $\begin{array}{llll}-1.10416700 & 1.57067000 & 0.61966800\end{array}$ $\begin{array}{llll}-1.16670500 & 3.01782600 & -0.96465900\end{array}$ $\begin{array}{llll}3.74588000 & 4.29469500 & 1.80373100\end{array}$ $\begin{array}{llll}5.30255700 & 2.53789800 & 1.26412700\end{array}$ $\begin{array}{llll}4.50785700 & 1.24826400 & -0.62569000\end{array}$ $\begin{array}{llll}4.20633400 & 0.38464100 & 1.45909400\end{array}$ $\begin{array}{lll}-0.00941000 & 5.52380100 & 1.02690900\end{array}$ $\begin{array}{lll}1.37405600 & 5.86214700 & 2.06328800\end{array}$ $\begin{array}{llll}0.02635500 & 4.90459400 & 2.67024200\end{array}$ $\begin{array}{llll}-2.23185600 & 2.88600200 & -1.14334200\end{array}$ $\begin{array}{llll}-0.90951900 & 4.06104600 & -1.14088800\end{array}$ $\begin{array}{llll}-0.62579500 & 2.41126600 & -1.68800800\end{array}$ $\begin{array}{llll}5.03861100 & 3.80721300 & 1.75755300\end{array}$ $\begin{array}{llll}3.57063100 & 5.28093800 & 2.20479800\end{array}$ $\begin{array}{lll}6.62412100 & 2.33144100 & 1.34688500\end{array}$ $\begin{array}{llll}6.26054300 & 4.39774100 & 2.15143000\end{array}$ $\begin{array}{llll}7.25659800 & 3.46993800 & 1.88551100\end{array}$ $\begin{array}{llll}7.28872800 & 1.08059600 & 0.88908300\end{array}$ $\begin{array}{llll}6.44735400 & 5.75889600 & 2.72966600\end{array}$ $\begin{array}{llll}8.72121800 & 3.61156200 & 2.15262200\end{array}$ $\begin{array}{lll}6.62051400 & 0.23461100 & 1.02765200\end{array}$ $\begin{array}{llll}8.18207100 & 0.91378500 & 1.48685800\end{array}$ $\begin{array}{llll}7.70654000 & 1.18041200 & -0.59547300\end{array}$ $\begin{array}{llll}5.49758600 & 6.25238300 & 2.91334100\end{array}$ $\begin{array}{llll}7.02861200 & 6.38564700 & 2.05404300\end{array}$ $\begin{array}{llll}6.98931900 & 5.70480400 & 3.67245700\end{array}$ $\begin{array}{llll}9.29046700 & 3.09146200 & 1.38462400\end{array}$ $\begin{array}{llll}8.99261200 & 4.66515700 & 2.08845800\end{array}$ $\begin{array}{llll}9.11529000 & 3.06447300 & 3.52670400\end{array}$ $\begin{array}{llll}8.36211000 & -0.07786300 & -1.04507800\end{array}$ $\begin{array}{llll}8.37991900 & 2.02234000 & -0.72944400\end{array}$ $\begin{array}{llll}6.81487500 & 1.35377500 & -1.19274200\end{array}$ $\begin{array}{llll}8.87354400 & 2.00573400 & 3.59934000\end{array}$ $\begin{array}{llll}10.18335300 & 3.18192100 & 3.69802700\end{array}$ $\begin{array}{llll}8.57702000 & 3.58466600 & 4.31745400\end{array}$ $\begin{array}{llll}7.71819900 & -1.27315100 & -1.43474200\end{array}$ $\begin{array}{llll}9.68875200 & -0.23939800 & -1.08861500\end{array}$ $\begin{array}{llll}8.71403400 & -2.18672600 & -1.73872500\end{array}$ $\begin{array}{llll}6.24096500 & -1.48752700 & -1.51696100\end{array}$ $\begin{array}{llll}9.94990800 & -1.53290100 & -1.51781900\end{array}$ $10.77297000 \quad 0.80431400 \quad-0.70702000$ $\begin{array}{llll}8.52041500 & -3.59234100 & -2.19601000\end{array}$ $\begin{array}{llll}6.03045900 & -2.54168100 & -1.33708700\end{array}$ $\begin{array}{llll}5.74093300 & -0.92659600 & -0.73022700\end{array}$ $\begin{array}{llll}5.65099400 & -1.07283700 & -2.86617100\end{array}$ $11.24684400-1.98625100-1.65236900$ $\begin{array}{llll}12.14738900 & 0.11120300 & -0.89339700\end{array}$ $\begin{array}{llll}10.61451200 & 1.19728600 & 0.62584200\end{array}$ $\begin{array}{llll}10.68081400 & 1.92591800 & -1.53263300\end{array}$ $\begin{array}{llll}7.92104900 & -3.62237800 & -3.10460900\end{array}$ $\begin{array}{llll}9.46762500 & -4.08313600 & -2.39894100\end{array}$ $\begin{array}{llll}7.99491700 & -4.17217500 & -1.43791200\end{array}$ $\begin{array}{llll}6.10711100 & -1.63689800 & -3.67816000\end{array}$ $\begin{array}{llll}4.57614500 & -1.24541800 & -2.88249900\end{array}$ $\begin{array}{llll}5.82512200 & -0.01481900 & -3.04918200\end{array}$ $12.33159000-1.18417000-1.35039700$ $\begin{array}{llll}11.41792100 & -2.99524600 & -1.99415200\end{array}$ $\begin{array}{llll}13.35014800 & 0.64268100 & -0.65074500\end{array}$ $\begin{array}{llll}13.71908600 & -1.44782700 & -1.39381400\end{array}$ $\begin{array}{llll}14.35784000 & -0.29648500 & -0.95603400\end{array}$ $\begin{array}{llll}13.52139600 & 2.01730700 & -0.11161400\end{array}$ $\begin{array}{llll}14.36860500 & -2.71660900 & -1.83189500\end{array}$ $\begin{array}{llll}15.82860100 & -0.09393900 & -0.77895300\end{array}$ $\begin{array}{llll}12.83616600 & 2.70840600 & -0.59474600\end{array}$ $\begin{array}{lll}13.30428000 & 2.02929800 & 0.95639600\end{array}$ $\begin{array}{llll}14.54242700 & 2.35519100 & -0.26034200\end{array}$ $\begin{array}{llll}14.95514700 & -2.55633600 & -2.73600100\end{array}$

$\begin{array}{llll}\mathrm{H} & 15.04449600 & -3.08982200 & -1.06411700 \\ \mathrm{H} & 13.63484500 & -3.48970900 & -2.04030700 \\ \mathrm{H} & 16.11164600 & 0.89897500 & -1.12834300 \\ \mathrm{H} & 16.36305900 & -0.80618000 & -1.40626900 \\ \mathrm{C} & 16.26271700 & -0.26599400 & 0.67821500 \\ \mathrm{H} & 16.01270400 & -1.26415900 & 1.03310100 \\ \mathrm{H} & 17.33510900 & -0.11739400 & 0.78848100 \\ \mathrm{H} & 15.74932000 & 0.45132000 & 1.31615700\end{array}$

Geometry: M052X-D3/def2-TZVP/toluene (PCM) Frequency: M052X-D3/def2-SVP/toluene (PCM)

$\begin{array}{ll}\text { Zero-point correction }= & 1.633426 \\ \text { Thermal correction to Energy= } & 1.735794 \\ \text { Thermal correction to Enthalpy= } & 1.736738 \\ \text { Thermal correction to Gibbs Free Energy= } & 1.477111 \\ \text { Sum of electronic and zero-point Energies= } & -4377.865233 \\ \text { Sum of electronic and thermal Energies }= & -4377.762866 \\ \text { Sum of electronic and thermal Enthalpies= } & -4377.761922 \\ \text { Sum of electronic and thermal Free Energies }= & -4378.021548\end{array}$

Single Point: DSD-BLYP-D3/def2-TZVPP/toluene (SMD)

Dispersion correction $\quad-0.172928611$

FINAL SINGLE POINT ENERGY $\quad-4380.209397672579$

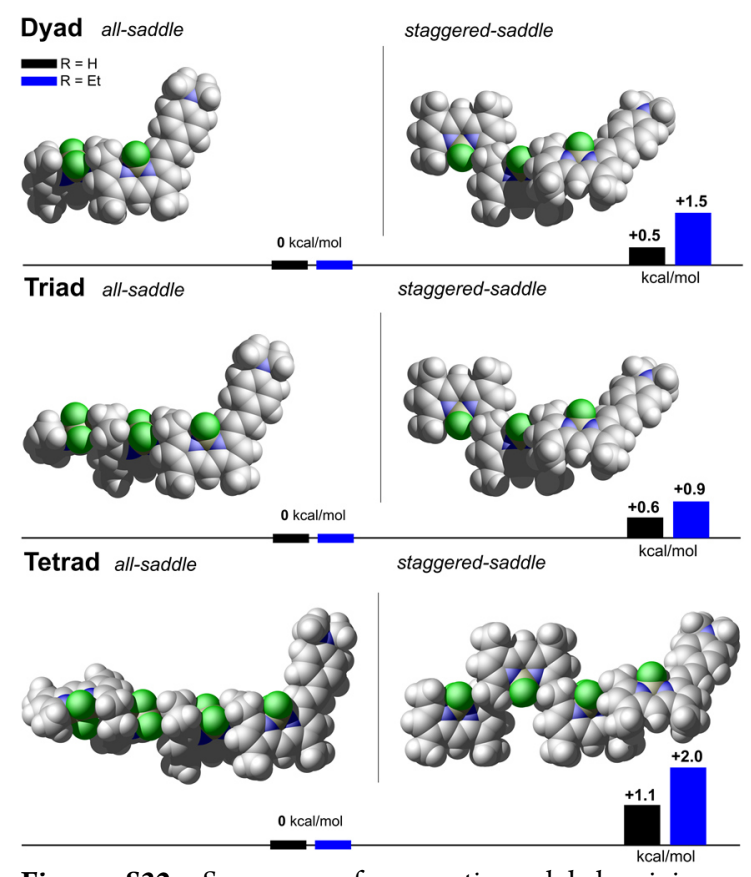

Figure S32. Summary of competing global minimum structures and corresponding final energies (exemplarily depicted for the $\beta$-unsubstituted series (DM)). 


\subsection{Energy Transfer rates}

In the following, we consider the red BODIPY unit as an acceptor system and the whole green BODIPY unit consistently as the donor system. All quantities were calculated at the wB97XD/def2-TZVPP level using the polarizable continuum model (IEFPCM) with toluene as solvent.

\section{Total electronic coupling}

In the case of a molecular system that consists of a donor and acceptor the diabatic Hamiltonian is given by

$$
H_{D-A}=\left(\begin{array}{cc}
E_{A} & V \\
V & E_{D}
\end{array}\right)
$$

Here $E_{A} / E_{D}$ are the excitation energies of the acceptor and donor, respectively and $V$ is the electronic coupling between both chromophores. We computed the electronic excited states of the isolated donor and acceptor as well as of the donor-acceptor dimer at the TD-DFT level. The coupling is calculated by

$$
V^{\mathrm{TD}-\mathrm{DFT}}=\sqrt{\left(\frac{\delta E}{2}\right)^{2}-\left(\frac{\Delta E_{\mathrm{D}-\mathrm{A}}}{2}\right)^{2}} .
$$

Where $\delta E$ denotes the splitting of the first two adiabatic excited states in the dimer and $\Delta E_{\mathrm{D}-\mathrm{A}}$ is the difference in the excitation energies of the donor and acceptor system.

\section{Coulomb contribution to the electronic coupling}

Transition density cube method (TDC). The coulomb part of the electronic coupling between two chromophores can be calculated as

$$
V^{\mathrm{Coul}}=\frac{1}{4 \pi \epsilon} \int d \boldsymbol{r} d \boldsymbol{r}^{\prime} \frac{\rho_{e g}^{A}(\boldsymbol{r}) \rho_{e g}^{B}\left(\boldsymbol{r}^{\prime}\right)}{\left|\boldsymbol{r}-\boldsymbol{r}^{\prime}\right|}
$$

Here $\rho_{\text {eg }}^{X}$ denotes the transition density between the ground state and the first excited state on the chromophore $X \in\{A, B\}$. As Shown by Scholes et al. evaluation of the Coulomb integral can be done by integration on a three-dimensional grid of finite-sized volume elements. ${ }^{10}$

Transition charges from electrostatic potential (TrEsp). The TrEsp procedure introduced by Renger et al. but also previously discussed by Scholes et al. uses atom centered transition charges that are fitted in order to reproduce the electrostatic potential of the electronic transition density of the monomer. ${ }^{10,11}$ This allows to compute the electronic coupling from

$$
V^{\text {Coul }}=\frac{1}{4 \pi \epsilon} \sum_{i, j} \frac{q_{\mathrm{eg}, i,}^{\mathrm{A}} q_{\mathrm{eg} . j}^{\mathrm{B}}}{\left|\mathbf{R}_{i}^{A}-\mathbf{R}_{j}^{\mathrm{B}}\right|}
$$

Here $q_{\mathrm{eg}, i}^{\mathrm{A}}$ are the atom-centered transition charges on atom $i$ from monomer $A$ and $\mathbf{R}_{i}^{A}$ is the atomic coordinate of atom $i$ from monomer $A$. 
Multipole expansion: Dipole-Dipole interaction. Multipole expansion around the molecular center of charge allows to approximate the Coulomb coupling as a dipole-dipole interaction:

$$
V^{\text {Coul }}=\frac{1}{4 \pi \epsilon}\left(\frac{\mu_{e g}^{A} \cdot \mu_{e g}^{B}}{R_{A B}^{3}}-3 \frac{\left(\mu_{e g}^{A} \cdot \mathbf{R}_{A B}\right)\left(\mathbf{R}_{A B} \cdot \boldsymbol{\mu}_{e g}^{B}\right)}{R_{A B}^{5}}\right)
$$

By introducing the angles $\cos \Phi=\frac{\mu_{e g}^{A} \cdot \mu_{e g}^{B}}{\left|\mu_{e g}^{A}\right| \cdot\left|\mu_{e g}^{B}\right|}, \cos \Theta_{A}=\frac{\mu_{e g}^{A} \cdot R_{A B}}{\left|\mu_{e g}^{A}\right| \cdot\left|R_{A B}\right|}, \cos \Theta_{B}=\frac{\mu_{e g}^{B} \cdot R_{A B}}{\left|\mu_{e g}^{B}\right| \cdot\left|R_{A B}\right|}$ on can write the Coulomb coupling also as:

$$
V^{\mathrm{Coul}}=\frac{\left|\boldsymbol{\mu}_{e g}^{A}\right|\left|\boldsymbol{\mu}_{e g}^{B}\right|}{4 \pi \epsilon R_{A B}^{3}} \underbrace{\left(\cos \Phi-3 \cos \Theta_{A} \cos \Theta_{B}\right)}_{\kappa}
$$

This distance, $R_{A B}$, between the charge centers of the individual chromophores has been calculated according to:

$$
R_{A B}=\left|\frac{\int\left|\rho_{e g}^{A}(\vec{r})\right| \cdot \vec{r} d \vec{r}}{\int d \vec{r}\left|\rho_{e g}^{A}(\vec{r})\right|}-\frac{\int\left|\rho_{e g}^{B}(\vec{r})\right| \cdot \vec{r} d \vec{r} \mid}{\int d \vec{r}\left|\rho_{e g}^{B}(\vec{r})\right|}\right|
$$

\section{Scaling scheme}

As in detail discussed by Scholes et al. it is well known that methods that only account for single excitations like CIS/TD-DFT significantly overestimate the magnitude of electronic transition moments. ${ }^{12}$ Therefore we use the scaling scheme from the paper of Scholes et al. ${ }^{12}$ in order to correct for this. The transition densities, transition charges and transition dipoles were rescaled by the ratio of the experimental absorption transition dipole moments to the TDDFT ones. The values as well as the scaling factors, $\alpha$, are given in the following table.

Table S2. Experimental and computed TD-DFT transition dipole moments for the individual fragments together with the scaling relation $\alpha$.

\begin{tabular}{rcccccc} 
& $\left|\mu_{\text {Red }}^{\text {exp }}\right|$ & $\left|\mu_{\text {Red }}^{\mathrm{TD}-\mathrm{DFT}}\right|$ & $\alpha_{\text {Red }}$ & $\left|\mu_{\mathrm{G}}^{\mathrm{exp}}\right|$ & $\left|\mu_{\mathrm{G}}^{\mathrm{TD}-\mathrm{DFT}}\right|$ & $\alpha_{\mathrm{G}}$ \\
\hline 1G-Red & 8.5 & 12.0 & 0.71 & 5.9 & 8.3 & 0.72 \\
2G-Red & 8.5 & 12.0 & 0.71 & 8.7 & 12.1 & 0.72 \\
3G-Red & 8.5 & 11.9 & 0.72 & 10.6 & 14.9 & 0.71 \\
\hline 1G-Red|Et & 8.4 & 11.9 & 0.71 & 5.9 & 8.8 & 0.67 \\
2G-Red|Et & 8.4 & 11.9 & 0.71 & 8.9 & 12.8 & 0.70 \\
3G-Red|Et & 8.4 & 11.7 & 0.72 & 11.0 & 15.5 & 0.71
\end{tabular}

We have computed the Coulomb couplings with these three different methods, to check if the multipole expansion that is used in the original Förster theory is applicable in these systems. Figure S33 shows the Coulomb interaction as a function of the number of green chromophores for both series. It is obvious, that coupling based on the dipole-dipole approximation overestimates the coupling in the dyads and underestimates it in the triads and tetrads. These values decrease by a factor of 2-3 by going from the dyad to the tetrad instead of 1.25 as obtained by the transition density cube method. 

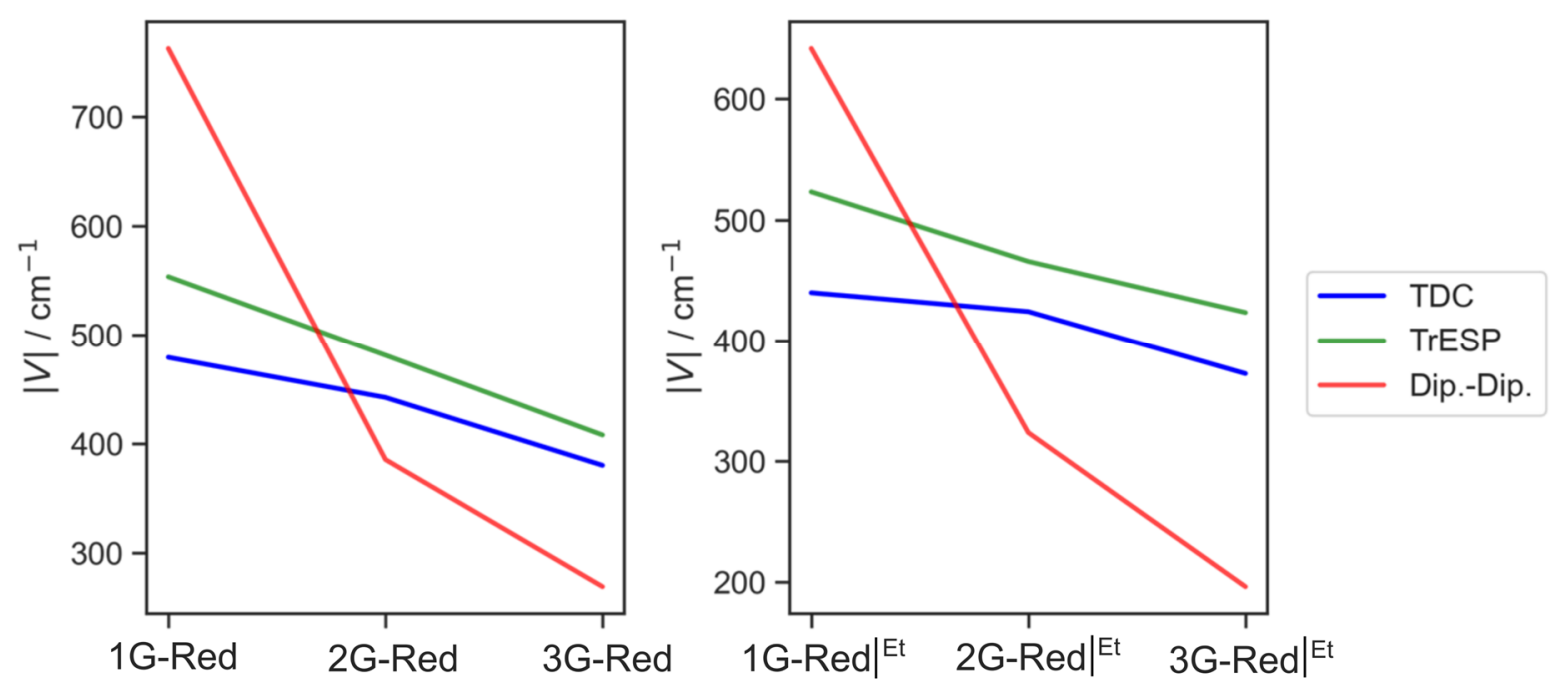

Figure S33. Coulomb couplings computed via the TDC method (transition density cube), via the TrESP method (transition charges from electrostatic potentials) and from transition dipole moments (Dip.-Dip.) as a function of the number of green chromophore units.

The total coupling consists of the Coulomb-coupling as well as of a short-range contribution that includes the exchange interaction as well as a penetration term

$$
V^{\mathrm{TD}-\mathrm{DFT}}=V^{\text {Coul,TD-DFT }}+V^{\text {short,TD-DFT }} .
$$

With the total coupling and the Coulomb coupling at hand we can estimate the short-range contribution. For this we followed exactly the procedure described by Scholes and Fleming. ${ }^{12}$ Which is given here for the sake of completeness:

1) Computation of the excited states of the dimer and of each monomer at the TD-DFT level

2) Calculation of the total coupling, $V^{\mathrm{TD}-\mathrm{DFT}}$, from the TD-DFT excited states

3) Calculation of the transition densities for each monomer and rescaling them so that the integral $\int d \boldsymbol{r} \rho_{e g}(\boldsymbol{r}) \boldsymbol{r}$ reproduces the TD-DFT transition dipole moments. Based on these transition

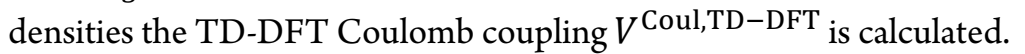

4) Determination of the short-range coupling, $V^{\text {short,TD-DFT }}=V^{\mathrm{TD}-\mathrm{DFT}}-V^{\text {Coul,TD-DFT }}$

5) Rescaling of $V^{\text {Coul,TD-DFT }}$ relative to the experimental transition moments, to get $V^{\text {Coul }}=$ $V^{\text {Coul,TD-DFT }} \alpha_{\text {Red }} \alpha_{\mathrm{G}}$.

The resulting values are given in Table S3 together with the experimental spectral overlap integrals. The molecular dyads show a short-range coupling, that is only about a factor of 2 smaller than the Coulomb coupling. Therefore, the interference term $2 V^{\text {Coul }} V^{\text {short }}$ in the energy transfer rates will be on the same order of magnitude as the transfer term mediated by the Coulomb coupling $\left|V^{\text {Coul }}\right|^{2}$.

This means that all approaches that only rely on the Coulomb part of the electronic coupling are not suitable for the accurate calculations of the energy transfer rates. 
Table S3: Computed couplings, charge center distances, orientation factors and spectral overlap integrals.

\begin{tabular}{|c|c|c|c|c|c|c|c|c|}
\hline & $\mathbf{V}^{\mathrm{TD}-\mathrm{DFT}} / \mathrm{cm}^{-1}$ & $\mathbf{V}_{\mathrm{TDC}}^{\text {Coul }} / \mathrm{cm}^{-1}$ & $\mathbf{V}_{\text {TrESP }}^{\text {Coul }} / \mathrm{cm}^{-1}$ & $\mathbf{V}_{\text {Kasha }}^{\text {Coul }} / \mathrm{cm}^{-1}$ & $\mathbf{V}^{\text {short }} / \mathrm{cm}^{-1}$ & $\mathbf{R}_{\mathrm{AB}} / \mathrm{m}$ & $\kappa$ & $\int \mathrm{d} \epsilon J /$ Hartree $^{-1}$ \\
\hline 1G-Red & -726.0 & -479.3 & -553.6 & -762.6 & 214.2 & $7.8 e^{-10}$ & 1.429 & 16.386 \\
\hline 2G-Red & -741.8 & -442.5 & -481.3 & -385.6 & 125.7 & $1.1 \mathrm{e}^{-9}$ & 1.396 & 25.134 \\
\hline 3G-Red & -671.8 & -380.3 & -408.1 & -269.3 & 76.8 & $1.4 \mathrm{e}^{-9}$ & 1.503 & 31.929 \\
\hline 1G-Red|Et & -704.8 & -439.8 & -523.3 & -641.8 & 214.7 & $8.1 \mathrm{e}^{-10}$ & 1.348 & 27.256 \\
\hline 2G-Red|Et & -745.7 & -424.1 & -465.7 & -323.5 & 112.3 & $1.1 \mathrm{e}^{-9}$ & 1.166 & 33.455 \\
\hline 3G-Red|Et & -700.2 & -372.4 & -423.4 & -196.1 & 29.3 & $1.4 \mathrm{e}^{-9}$ & 1.063 & 43.689 \\
\hline
\end{tabular}

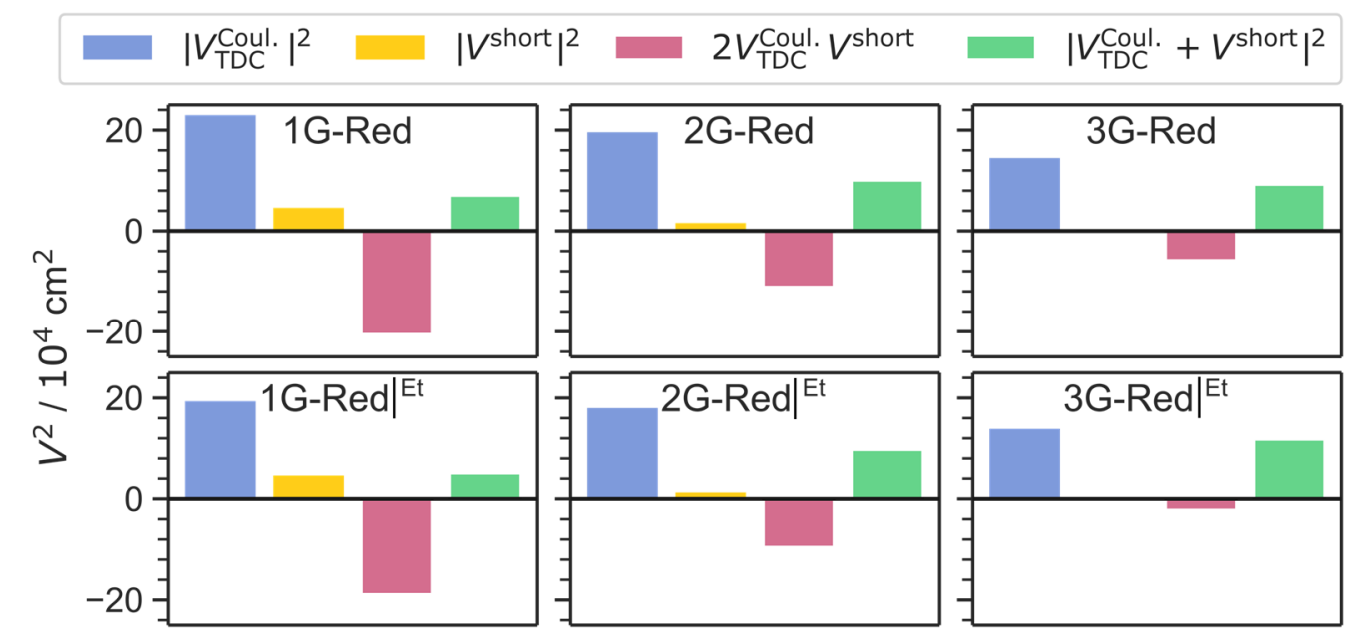

Figure S34. Computed coupling terms for the series of heterooligomers and their contributions to the energy transfer rate.

6.3 FRET rates according to traditional formula

$$
k_{r}=\frac{9 \kappa^{2} \ln 10}{128 \pi^{5} n^{4} N_{A} \tau_{D}} \cdot \frac{\Phi_{e}^{D}}{r^{6}} \int \epsilon_{A}(\lambda) I_{F}^{D}(\lambda) \lambda^{4} d \lambda
$$

Table S4: FRET rates $k_{\mathrm{FT}}$ and corresponding lifetimes $\tau_{\mathrm{FT}}$ calculated with traditional FRET equation in toluene $(n=1.4969)$. QY: quantum yield of fluorescence.

\begin{tabular}{rccccccc} 
& $\begin{array}{c}\text { lifetime } \tau_{\mathrm{FT}} \\
/ \mathrm{fs}\end{array}$ & $\begin{array}{c}k_{\mathrm{FT}} \\
/(1 / \mathrm{s})\end{array}$ & $\begin{array}{c}\text { spectral overlap } \\
\text { integral } \\
/\left(\mathrm{nm}^{4} / \mathrm{M} \cdot \mathrm{cm}\right)\end{array}$ & $\begin{array}{c}\text { QY of } \\
\text { Donor } \Phi_{e}{ }^{D}\end{array}$ & $\begin{array}{c}\text { lifetime of } \\
\text { Donor } \tau_{D} \\
/(1 / \mathrm{s})\end{array}$ & $\boldsymbol{r} / \mathrm{m}$ & $\kappa$ \\
\hline 1G-Red & $\mathbf{2 7}$ & $3.65 \mathrm{e}^{13}$ & $1.24 \mathrm{e}^{15}$ & 0.86 & $4.63 \mathrm{e}^{-9}$ & $7.8 \mathrm{e}^{-10}$ & 1.429 \\
2G-Red & $\mathbf{1 0 4}$ & $9.64 \mathrm{e}^{12}$ & $1.91 \mathrm{e}^{15}$ & 0.81 & $3.09 \mathrm{e}^{-9}$ & $1.1 \mathrm{e}^{-9}$ & 1.396 \\
3G-Red & $\mathbf{1 5 5}$ & $6.47 \mathrm{e}^{12}$ & $2.48 \mathrm{e}^{15}$ & 0.86 & $1.73 \mathrm{e}^{-9}$ & $1.4 \mathrm{e}^{-9}$ & 1.503 \\
\hline 1G-Red|Et & $\mathbf{2 4}$ & $4.23 \mathrm{e}^{13}$ & $2.26 \mathrm{e}^{15}$ & 0.86 & $5.17 \mathrm{e}^{-9}$ & $8.1 \mathrm{e}^{-10}$ & 1.348 \\
2G-Red|Et & $\mathbf{1 0 1}$ & $9.92 \mathrm{e}^{12}$ & $2.69 \mathrm{e}^{15}$ & 0.84 & $3.06 \mathrm{e}^{-9}$ & $1.1 \mathrm{e}^{-9}$ & 1.166 \\
3G-Red|Et & $\mathbf{3 1 9}$ & $3.14 \mathrm{e}^{12}$ & $3.43 \mathrm{e}^{15}$ & 0.84 & $2.41 \mathrm{e}^{-9}$ & $1.4 \mathrm{e}^{-9}$ & 1.063
\end{tabular}


6.4 Field-induced surface hopping dynamics simulations

The idea of the field-induced surface hopping (FISH) method is to propagate independent classical trajectories in a manifold of several electronic states and to describe the population transfer between them, due to coupling with the laser field and due to nonadiabatic coupling, by allowing the trajectories to switch between the states. The theoretical foundations of FISH have been presented at full length in refs. ${ }^{13,14}$ In the FISH simulations, an ensemble of classical trajectories, representing a nuclear wavepacket, is propagated in a manifold of three adiabatic electronic states and the population transfer between these states is described by a stochastic hopping procedure, which is based on the change of the quantum electronic state populations..$^{13,15}$ The ground-state phase space distribution, from which the initial conditions were sampled has been approximated by a canonical harmonic-oscillator Wigner distribution: ${ }^{16}$

$$
\rho_{00}\left(\mathbf{q}_{0}, \mathbf{p}_{0}\right)=\prod_{n=1}^{N_{\text {modes }}} \frac{\alpha_{n}}{\pi \hbar} \exp \left[-\frac{\alpha_{n}}{\hbar \omega_{n}}\left(p_{0 n}^{2}+\omega^{2} q_{0 n}^{2}\right)\right]
$$

Here $q_{0 n}$ and $p_{0 n}$ denote the ground state normal coordinates and the conjugate momenta. The temperature dependency is given by the quantitiy $\alpha=\tanh \left(\frac{\hbar \omega_{n}}{2 k_{b} T}\right)$. The 150 initial coordinates and momenta have been generated by sampling the harmonic Wigner distribution at a temperature of $298 \mathrm{~K}$. In field-induced surface hopping dynamics simulations the classical Newton equations were integrated using the velocity Verlet algorithm with a time step of $0.2 \mathrm{fs}$ for the propagation of the nuclei. Along each trajectory, the electronic Schrödinger equation has been integrated using the fourth-order Runge-Kutta method with a time step of $8 \times 10^{-5}$ fs. In our simulation, we have performed nonadiabatic surface hopping molecular dynamics in the manifold of the ground state and the lowest four excited singlet states. In this manner, time-dependent electronic state populations are obtained, which are then used to calculate surface-hopping probabilities. ${ }^{17}$

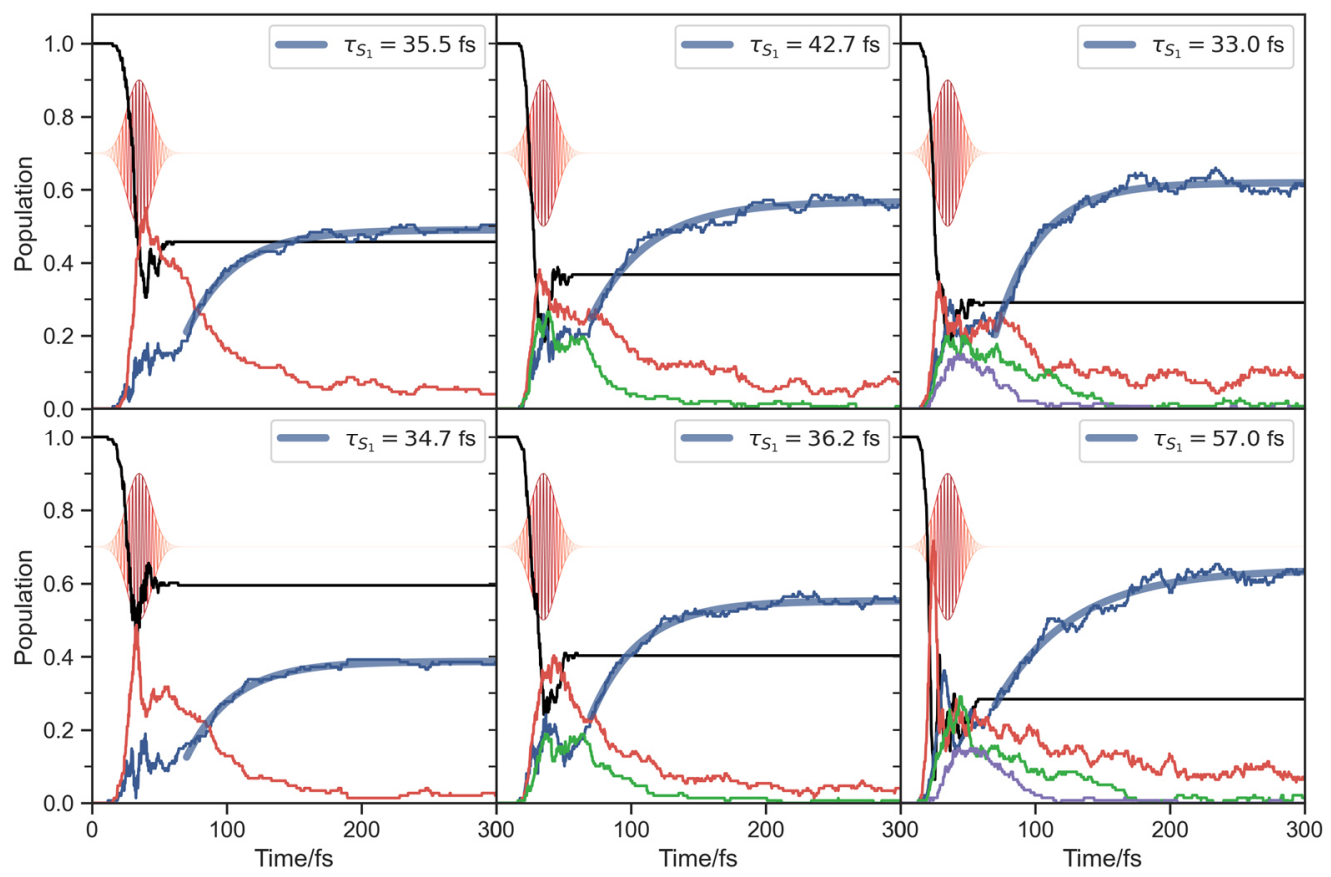

Figure S35. Time dependent electronic state populations for an ensemble of 140 trajectories during the field-induced surface hopping simulations of all six BODIPY systems. In the upper panel the DM series 1G-Red (left), 2G-Red (middle) and 3G-Red (right) is shown. While in the lower panel the EDM series with 1G-Red $\left.\right|^{\mathrm{Et}}$ (left), 2G-Red $\left.\right|^{\mathrm{Et}}$ (middle) and 3G-Red| $\left.\right|^{\mathrm{Et}}$ (right) is depicted. The bold blue line shows the fit of the $S_{1}$ populations to the following fit function:

$\mathrm{G}(\mathrm{t})=\mathrm{A} \exp \left(-\frac{\mathrm{t}-\mathrm{t}_{0}}{\tau}\right) \exp \left(\frac{\mathrm{b}^{2}}{4 \sigma^{2}}\right)\left[1+\operatorname{erf}\left(\frac{\mathrm{t}-\mathrm{t}_{0}-\frac{\mathrm{b}^{2}}{2 \sigma}}{\mathrm{b}}\right)\right]$. Here $\tau$ is the time constant for the population rise. The values of the time constants are shown in the inset and the time evolution of the laser pulse is shown in the background. 


\section{References}

(1) Van Stokkum, I. H. M.; Larsen, D. S.; Van Grondelle, R. Biochim. Biophys. Acta, Bioenerg. 2004, 1657, 82.

(2) Snellenburg, J. J.; Laptenok, S. P.; Seger, R.; Mullen, K. M.; Stokkum, I. H. M. v. J. Stat. Software 2012, $49,1$.

(3) Steeger, M. Ph.D. Thesis, University of Würzburg, 2014.

(4) Gaussian 09, Revision D.01, Frisch, M. J.; Trucks, G. W.; Schlegel, H. B.; Scuseria, G. E.; Robb, M. A.; Cheeseman, J. R.; Scalmani, G.; Barone, V.; Mennucci, B.; Petersson, G. A.; Nakatsuji, H.; Caricato, M.; Li, X.; Hratchian, H. P.; Izmaylov, A. F.; Bloino, J.; Zheng, G.; Sonnenberg, J. L.; Hada, M.; Ehara, M.; Toyota, K.; Fukuda, R.; Hasegawa, J.; Ishida, M.; Nakajima, T.; Honda, Y.; Kitao, O.; Nakai, H.; Vreven, T.; Montgomery, J. A.Jr.; Peralta, J. E.; Ogliaro, F.; Bearpark, M.; Heyd, J.J.; Brothers, E.; Kudin, K. N.; Staroverov, V. N.; Keith, T.; Kobayashi, R.; Normand, J.; Raghavachari, K.; Rendell, A.; Burant, J. C.; Iyengar, S. S.; Tomasi, J.; Cossi, M.; Rega, N.; Millam, J. M.; Klene, M.; Knox, J. E.; Cross, J. B.; Bakken, V.; Adamo, C.; Jaramillo, J.; Gomperts, R.; Stratmann, R. E.; Yazyev, O.; Austin, A. J.; Cammi, R.; Pomelli, C.; Ochterski, J. W.; Martin, R. L.; Morokuma, K.; Zakrzewski, V. G.; Voth, G. A.; Salvador, P.; Dannenberg, J. J.; Dapprich, S.; Daniels, A. D.; Farkas, O.; Foresman, J. B.; Ortiz, J. V.; Cioslowski J. and D. J. Fox, Gaussian, Inc., Wallingford CT, 2013.

(5) (a) Grimme. S.; Antony. J.; Ehrlich. S.; Krieg. H. J. Chem. Phys. 2010, 133, 154104. (b) Grimme. S.; Ehrlich. S.; Goerigk. L. J. Comput. Chem. 2011 , 32, 1456.

(6) Marenich, A. V.; Cramer, C. J.; Truhlar, D. G. J. Phys. Chem. B 2009, 113, 6378.

(7) Neese, F. WIREs Comput. Mol. Sci. 2012, 2, 73.

(8) Patra, A.; Patalag, L. J.; Jones, P. G.; Werz, D. B. Angew. Chem. Int. Ed. 2021, 60, 747.

(9) Völker, S. F.; Schmiedel, A.; Holzapfel, M.; Renziehausen, K.; Engel, V.; Lambert, C. J. Phys. Chem. C 2014, 118, 17467.

(10) Krueger, B. P.; Scholes, G. D.; Fleming, G. R. J. Phys. Chem. B 1998, 102, 5378.

(11) Madjet, M. E.; Abdurahman, A.; Renger, T. J. Phys. Chem. B. 2006, 110, 17268.

(12) Scholes, G. D.; Gould, I. R.; Cogdell, R. J.; Fleming, G. R. J. Phys. Chem. B 1999, 103, 2543.

(13) Mitric, R.; Petersen, J.; Bonacic-Koutecky, V. Phys. Rev. A: At., Mol., Opt. Phys. 2009, 79, 053416/1.

(14) Mitric, R.; Werner, U.; Wohlgemuth, M.; Seifert, G.; Bonacic-Koutecky, V. J. Phys. Chem. A 2009, 113, 12700.

(15) Tully, J. C. J. Chem. Phys. 1990, 93, 1061.

(16) Wigner, E. Phys. Rev. 1932, 40, 749.

(17) Werner, U.; Mitric, R.; Suzuki, T.; Bonacic-Koutecky, V. Chem. Phys. 2008, 349, 319. 